\title{
Transmission Geometry X-ray \\ Diffraction for Materials Research
}

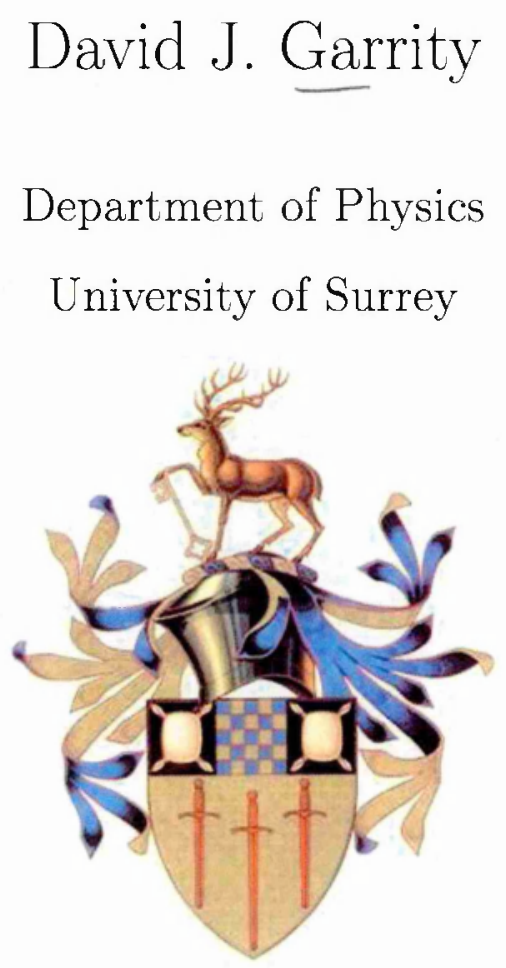

Submitted for the degree of Doctor of Philosophy

September 2007 
ProQuest Number: 27558533

All rights reserved

INFORMATION TO ALL USERS

The quality of this reproduction is dependent upon the quality of the copy submitted.

In the unlikely event that the author did not send a complete manuscript and there are missing pages, these will be noted. Also, if material had to be removed, a note will indicate the deletion.

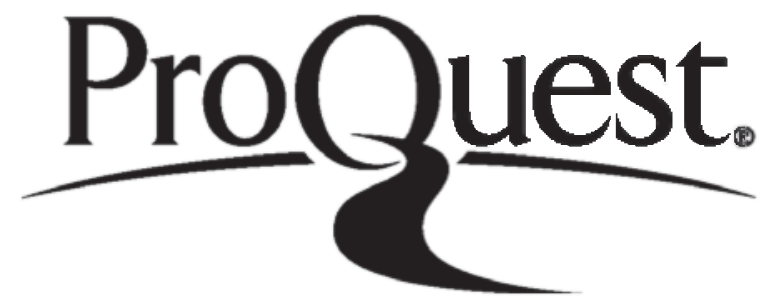

ProQuest 27558533

Published by ProQuest LLC (2019). Copyright of the Dissertation is held by the Author.

All rights reserved.

This work is protected against unauthorized copying under Title 17, United States Code Microform Edition (C) ProQuest LLC.

ProQuest LLC.

789 East Eisenhower Parkway

P.O. Box 1346

Ann Arbor, Ml $48106-1346$ 
In memory of my late father, Alan David Garrity. For you, Ad vitam aeternam... 
This work has been made possible thanks to Dr. Ian Giles and is funded by the Defence Procurement Agency (DPA)

(C) British Crown Copyright 2007/MOD

Any views expressed in this work are those of the author and do not represent those of Her Majesty's Government. 


\section{Abstract}

$\mathrm{X}$-ray diffraction is usually thought of as a surface analysis technique. However, given sufficiently energetic X-rays to overcome the inherent attenuation of the sample, this technique may be used for studying the nature of bulk materials. In the work presented here, transmission geometry X-ray diffraction has been further developed for imaging phase transformations and lattice spacing changes in low-alloy ferritic, and austenitic stainless steels under conditions of interest to the Naval Nuclear Propulsion Program (NNPP).

A broad account of X-ray diffraction in a transmission geometry is given, detailing the fundamentals of elastic scattering of X-rays including; the classically derived Thomson scatter cross section and the modified Rayleigh scattering cross section for scattering from individual electrons and atoms respectively, and Bragg diffraction that results from scattering of $\mathrm{X}$-rays by ordered, periodic substances.

The experimental configurations and apparatus used for energy dispersive and angular dispersive configurations are discussed, and preliminary results for both techniques are presented. Further to this, measurements of both ferritic and austenitic stainless steel are included, the latter involving both static and dynamic measurements of lattice spacing changes induced under 
an applied tensile load, demonstrating the ability to observe changes as small as $0.75 \%$.

Finally significant work has been carried out with regards to imaging the structural changes brought about by the martensite phase transformation in austenitic stainless steel. In a compressively pre-loaded sample of steel, martensite has been observed at points of high plastic strain by observing the distinct differences in the X-ray diffraction spectra obtained before, and after quenching in liquid nitrogen. This verifies the Finite Element (FE) code used to model these changes, and opens up potential avenues of online-monitoring in the context of the NNPP. 


\section{Acknowledgements}

"tu ne cede malis sed contra audentior ito"

A friend and fellow PhD student once told me: "you don't ' $d o$ ' a PhD... you survive it..." and with my friends and colleagues here at Surrey, I have.

I would like to thank my supervisors, Dr. Paul Jenneson and Dr. Stuart Vincent for their tireless support, inspiration and understanding (and encouragement to lighten up!). I could not have asked for, or been given, better supervisors or mentors for the rollercoaster ride that is a $\mathrm{PhD}$, particularly when writing this thesis with a fractured kneecap and leg immobilised in a splint! To my co-supervisor at Surrey, Dr. Paddy Regan, many thanks for your enthusiasm, humour, and for keeping tabs on my progress despite having three 'reprobates' to supervise yourself. To my mother and sister Charlotte, thank you for believing in me and supporting me this far.

Of the staff, I would particularly like to thank Terry McCarthy at the Nuclear Department, HMS Sultan, for his endless patience and assistance with my crazy designs, modifications and complete re-designs of the apparatus to mount in front of Sultan's X-ray tube. Similarly to Steve Craig at Surrey for manufacturing the various collimators and pieces of equipment used with the Surrey X-ray tube. Also to James Wallbank and John William Brown at 
Surrey, for keeping the detectors up and running and helping me to stay sane through the constant equipment failures that seemed to characterise the last 6-8 months. To Dr. Mark. Wenman and Alexander Price at HMS Sultan, thank you for all your input and the opportunity to travel to the ESRF.

To my fellow PhD students and Surrey colleagues, I thank you for making Surrey a fun and interesting place; Fellow students and comrades-in-pain 'Tough Guys' - Nick 'Elf' and Adam 'Garnzy' (I'll be damned if don't make it past the start-line next time - "knee? no problem!..."), my fellow 'spanner monkey', tea addict and land-boarding wingman Simon ("What you need, is something bigger...a wrench"). Thanks to everyone in my office/group, past and present, for making for such an interesting working environment; Chris for putting up with me as a desk neighbour and mate these last three years, Jamie (cheers for looking out for me), Tamara (thanks for all the help with LaTeX), Julian, Gareth ("This physics doesn't find itself"), Steve A (thanks the computer help and drubbings at pool), Steve S and Ian (cheers for the squash coaching guys), Nick P, Amanda ('anti-circuits'), Amy, David, Natasha, Julia, Jeff (circuit-training buddy and proof there is light at the end of the tunnel), Ed, Greg, Simon B, Elizabeth (cake-club' Overlord), Scott (thanks for the poker league even though I'm still bottom of it!), Andrew, Annika, Eric 'Ric' (fellow Tough Guy), James, and last but not least to the newest members of the PhD crew; Emma and Sam, for endless laughs over the last year and assistance with 'lightening up'.

\section{Cheers,}

\section{Dangerous}




\section{Contents}

1 Introduction 1

1.1 Background ..................... 1

1.2 Motivation/objectives of current work . . . . . . . . . 4

2 Elastic Scattering of X-rays $\quad 8$

2.1 Introduction . . . . . . . . . . . . 8

2.2 Thomson scatter from a free electron . . . . . . . . . 9

2.3 Rayleigh scatter from bound electrons . . . . . . . . . . . . 12

2.4 Bragg diffraction . . . . . . . . . . . . 16

3 Experimental configurations and apparatus $\quad 21$

3.1 Introduction . . . . . . . . . . . . . . . . . 21

3.2 Experiment configurations . . . . . . . . . . . 23

3.2.1 Energy dispersive apparatus . . . . . . . . . . 28

3.2 .2 Angular dispersive apparatus . . . . . . . . . 37

3.3 X-ray tubes . . . . . . . . . . . . . . . . . 39

3.3 .1 Production of X-rays . . . . . . . . . . . 39

3.3.2 Construction of X-ray tubes . . . . . . . . . . 42

vii 
3.4 Detectors . . . . . . . . . . . . . . 45

3.4.1 Introduction to germanium and other semiconductors . 45

3.4.2 Devices used for energy dispersive XRD . . . . . . 49

3.4.3 Devices used for angular dispersive XRD . . . . . . . 52

4 Experimental results and discussion 53

4.1 Introduction . . . . . . . . . . . . . . 53

4.2 Angular dispersive X-ray diffraction results . . . . . . . . 55

4.3 Energy dispersive X-ray diffraction results . . . . . . . 57

4.3.1 Energy dispersive XRD on low density materials . . . 57

4.3.2 Energy dispersive XRD on steel . . . . . . . . 61

4.3.3 Martensite phase transformation . . . . . . 77

4.3.4 Dynamic stress measurements using EDXRD . . . . . . 84

4.4 High energy diffraction . . . . . . . . . . . . 87

5 Outlook and future work $\quad 91$

5.1 Introduction . . . . . . . . . . . . . . 91

5.2 Future equipment development . . . . . . . . . . . 92

5.3 Further investigation ................ . 96

6 Conclusions 100

A $225 \mathrm{kV}$ X-ray Tube 104

$\begin{array}{ll}\text { B Phase transformation in steel } & 109\end{array}$

C Further developments in transmission XRD 114 


\section{List of Figures}

1.1 Previous proof of concept for the imaging of ferrite and austenite phases in steel . . . . . . . . . . . . 6

2.1 Variables involved in deriving the Thomson scatter cross section 11

2.2 Derivation of the momentum transfer for an elastically scattered photon . . . . . . . . . . . . . . . 13

2.3 Geometric considerations for deriving the Bragg equation . . . 17

2.4 Derivation of the phase factor for Bragg diffraction . . . . . 18

3.1 Schematic of a typical energy dispersive configuration. . . . . . 24

3.2 Example of an angular dispersive configuration . . . . . . . . 26

3.3 Example of the differential attenuation effects in increasing thicknesses of mild steel . . . . . . . . . . . . . 27

$3.4 \mathrm{X}$-ray beam filtration to produce a quasi-monochromatic beam 28

3.5 Photograph of energy dispersive X-ray diffraction apparatus . 29

3.6 Updated energy dispersive XRD apparatus . . . . . . . . . 32

3.7 Stepper-motor system . . . . . . . . . . . . . 33

3.8 Transmission shield wall . . . . . . . . . . . . . . 34

3.9 Dynamic stress apparatus at HMS Sultan . . . . . . . . 35 
3.10 Dynamic stress apparatus at Surrey . . . . . . . . . . 36

3.11 Schematic of the apparatus used for angular dispersive X-ray diffraction measurements. . . . . . . . . 38

3.12 Spectrum produced by an X-ray tube running at an operating voltage of $160 \mathrm{kV} \ldots \ldots \ldots 4 . \ldots \ldots$

3.13 Simplified configuration of a stationary anode X-ray tube . . . 43

3.14 Configuration of planar and coaxial HPGe detectors . . . . . . 47

3.15 Amptek XR-100T CZT detector . . . . . . . . . . . . 50

$3.16 \mathrm{CZT} /$ Germanium photopeak efficiency . . . . . . . 51

3.17 Hamamatsu image intensifier . . . . . . . . . . . . . 52

4.1 Diffraction images produced by angular dispersive X-ray diffrac-

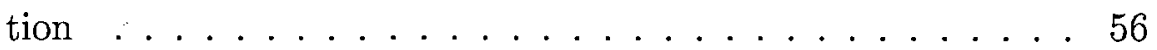

4.2 Energy dispersive spectra of sugar and $\mathrm{PVG} / \mathrm{SiO}_{2} \ldots \ldots$

4.3 Energy dispersive spectra of other materials . . . . . . . 59

4.4 Photograph of the sample holder used to heat sugar . . . . . 60

4.5 Intensity vs. energy plot for the region $27 \mathrm{keV}$ to $30 \mathrm{keV}$ for sugar at temperatures of $20^{\circ} \mathrm{C}$ through to $160^{\circ} \mathrm{C}$. . . . 60

4.6 Photograph of the steel samples used for EDXRD . . . . . . 62

4.7 Spectra obtained from ferrite-phase steel at scattering angles of $3^{\circ}$ through to $7^{\circ} \ldots \ldots \ldots \ldots$

4.8 Spectra obtained from ferrite-phase steel at scattering angles of $8^{\circ}$ through to $12^{\circ} \ldots \ldots \ldots \ldots 4$

4.9 Spectra obtained from austenite-phase steel at scattering angles of $3^{\circ}$ through to $7^{\circ} \ldots \ldots \ldots \ldots$ 
4.10 Spectra obtained from austenite-phase steel at scattering angles of $8^{\circ}$ through to $12^{\circ} \ldots \ldots \ldots 6$

4.11 Effect of differential attenuation . . . . . . . . . 67

4.12 Photograph of the portable stressing apparatus at HMS Sultan's Nuclear Department . . . . . . . . . . . . 68

4.13 Spectra obtained from plastically deformed austenite-phase steel 69

4.14 Lattice spacing data for austenite-phase steel samples . . . . . 70

4.15 Lattice spacing data for $26.5 \mathrm{~cm}$ austenite-phase steel sample . 70

4.16 Slip deformation of a crystalline structure . . . . . . . . 71

4.17 Comparison of stressed and unstressed austenite-phase steel . 73

4.18 Austenite structure and the martensite transformation . . . . 74

4.19 SEM images of plastically deformed stainless steel samples . . 75

4.20 Comparison of HPGe and CZT detector . . . . . . . . 76

4.21 Model of the residual stresses in 304L stainless steel sample . . 79

$4.22304 \mathrm{~L}$ circular sample pre-quenching . . . . . . . . . . 81

$4.23304 \mathrm{~L}$ circular sample post-quenching . . . . . . . . . 82

4.24 Pre and post quench lattice spacing intensities . . . . . . . 83

4.25 Dynamic stress measurements in 304L stainless steel . . . . . 85

4.26 Possible improvement to dynamic measurements . . . . . . . 87

4.27 High energy diffraction experiment at the ESRF . . . . . . . 89

4.28 Verification of $\mathrm{FE}$ model . . . . . . . . . . . . . 90

5.1 Possible updated EDXRD apparatus . . . . . . . . . . . 94

5.2 Possible further developments of mapping the martensite phase transformation ................... 97 


\section{Chapter 1}

\section{Introduction}

\subsection{Background}

The use of X-rays for imaging purposes can be dated back to their discovery over a century ago. In the intervening period, the field of X-ray imaging has developed along two parallel lines; that of X-ray diffraction/X-ray crystallography from the pioneering work of Laue and later Bragg, and that of radiography illustrated by Röentgen's famous radiograph of his wife's hand [1].

In more recent years there has been a great deal of focus on exploiting coherent (Rayleigh) scattered X-rays for imaging purposes. Although the classical relationship for elastic scattering (an electromagnetic wave causes an electron to oscillate sympathetically producing a secondary wavelet of the same frequency) was discovered in the early 20th century by Thomson [2] and others, its potential in a transmission geometry has only come to light within the last twenty years [3]. 
The potential for coherent scattering was first realised following investigations into streaking effects in medical transmission CT images [3]. Imaging of water phantoms revealed this phenomenon to be the result of constructive interference between elastically scattered X-rays from neighbouring water molecules. Given the requirement for the scattered waves to be in phase, this phenomenon is strongly peaked in the forward (i.e. low angle) direction as at higher angles destructive interference increases. For crystalline structures with regular periodicity this scattering will manifest itself as a series of fringes as associated with classic Bragg diffraction and thus allowing measurement of the inter-atomic spacings. Since this discovery the technique, sometimes referred to as Low Angle X-ray Scattering (LAXS), has been exploited in a wide range of fields. In regards to security applications, a great deal of work has been carried out over the last ten years [4][5][6] into the development of systems and analysis procedures to detect small quantities of concealed organic explosives in passenger baggage, the challenge being to distinguish explosives from other low- $Z$ materials typical of a suitcase contents while maintaining a suitably small measurement time (of the order of seconds) and rate of false alarms. The same techniques used to detect plastic explosives are also suitable for the identification of narcotics concealed within passenger baggage [6].

The potential of coherently scattered X-rays is not limited to security concerns, the technique has also been used in medical applications including determining simulated trabecular bone mineral loss and cortical bone thinning through the imaging of bone phantoms [7] with coherently scattered X-rays. In terms of in-vivo measurements the difficulty becomes that 
of maintaining sufficient intensity of X-rays (the coherently scattered beam is weak compared to the transmitted beam) to perform the measurement while minimising the dose to the patient (given that the scattering is highly forward-peaked, it is difficult to separate from the transmitted beam).

Industrial applications have not been neglected either; coherent scattering provides a simple and robust method of on-line measurement of oil/water ratios of the fluids extracted from oilfields [8]. Similarly the technique shows potential for the detection of low- $\mathrm{Z}$ contaminants, such as plastics, in foodstuffs [9].

The applications and techniques considered generally rely on measuring scattering from one region of an object under investigation. Another novel form of imaging is that of using Rayleigh scattered X-rays for Computed Tomography [10]. Rather than using the linear attenuation differences through a sample to construct the image, the elastic scatter differential cross section (i.e. scatter intensity) is used, dramatically increasing the contrast compared to that of conventional transmission CT. More recently, development of this. technique has been focused in improving the identification of cancerous breast tissue from healthy tissue [11][12], illustrating the contrast improvement over transmission images.

The use of coherent scattering for imaging purposes, particularly that of coherent scatter CT (or X-ray diffraction CT), therefore represents a coming together of the two parallel methods of X-ray diffraction and imaging through radiography/CT. The areas of development described previously represent a small section of a growing number of applications for what is still a relatively young imaging technique. 


\subsection{Motivation/objectives of current work}

In a collaborative effort between the University of Surrey and the Nuclear Department at HMS Sultan, the materials research potential of transmission geometry X-ray diffraction (i.e. coherently scattered X-rays) in the context of the Naval Nuclear Propulsion Programme (NNPP) is to be investigated. The primary focus of this work is to investigate the imaging potential for environmentally induced factors including stress and corrosion in both lowalloy steels and austenitic stainless steals.

Steel [13], is a ferrous alloy consisting of an iron crystalline structure with varying quantities of interstitially dissolved carbon, where the percentage carbon content determines the final mechanical properties (such as the strength) of the steel. Such steels are known as 'plain carbon steels' and are sub-divided into low carbon $(<0.3 \% \mathrm{C})$, medium carbon $(0.3-0.7 \% \mathrm{C})$ and high carbon $(0.7-1.7 \% \mathrm{C})$ categories, where increasing the carbon content increases the strength of the steel at the expense of the ductility. Aside from the dissolved carbon, the presence of other elements in the form of impurities is minimised during manufacture of plain carbon steels.

Stainless steels such as the 304L alloy used for this work however, are 'doped' with specific quantities of additional elements such as nickel, chromium and manganese, in order to further tailor the mechanical properties (such as hardness and toughness) for the required application. Comparing the 304L stainless steel with plain carbon steels for example, this alloy has high ductility, high tensile strength, and good corrosion resistance rendering it particularly suitable for applications in the NNPP and Nuclear industry in general. 
The iron-carbon system that is steel, is further complicated in that several distinct solid phases exist, with different crystalline structures, including the ferrite $(\alpha)$ phase and austenite $(\gamma)$ phase [13]. As discussed further in section 4.3.3, another, metastable phase known as martensite can exist upon rapid cooling of austenitic steel, forming the basis of heat-treatments for further strengthening.

With regards to coherent X-ray scattering, this is now an established imaging tool, both as a complementary technique to conventional transmission techniques, and as a unique non-destructive testing method. Measurement of, for example, the energy of X-ray photons coherently scattered from a crystalline substance, produces a diffraction profile that consists of a series of sharply defined peaks. It is this diffraction profile, characteristic for a given substance, that allows for the discrimination of an organic explosive such as Semtex from a benign substance such as soap or deodorant in suitcases [4]. In such examples the measured diffraction profiles are radically different; organic explosives are crystalline in structure, generating a complicated series of sharp peaks while the deodorant being an amorphous substance, produces a single broad peak in the diffraction profile.

The generation of a diffraction profile is further useful in that the inter atomic spacings may be measured by the simple expedient of calibrating the profile in terms of the momentum transfer parameter $q$ (refer to section 2.3). This is not only useful in terms of identifying a material, but also provides an opportunity to monitor changes induced in a material due to changing environmental conditions.

In previous work [14], transmission geometry X-ray diffraction has been 


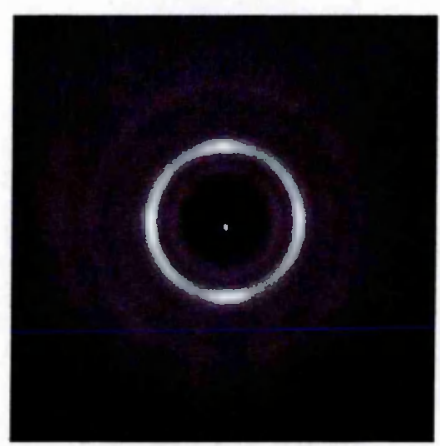

(a)

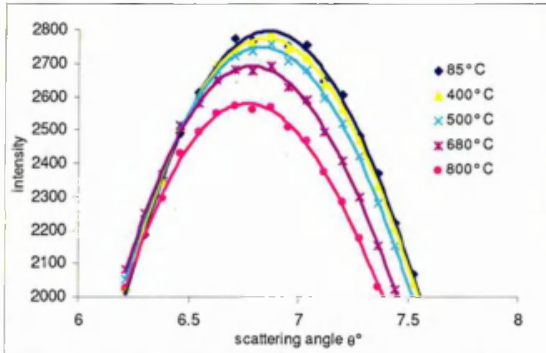

(c)

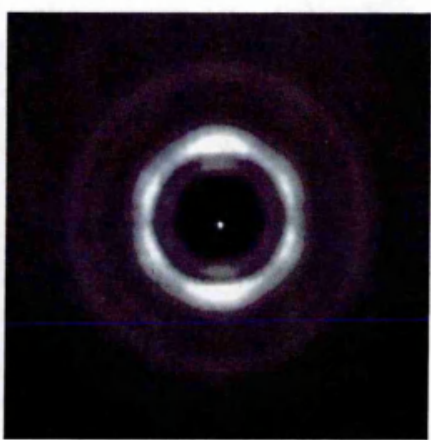

(b)

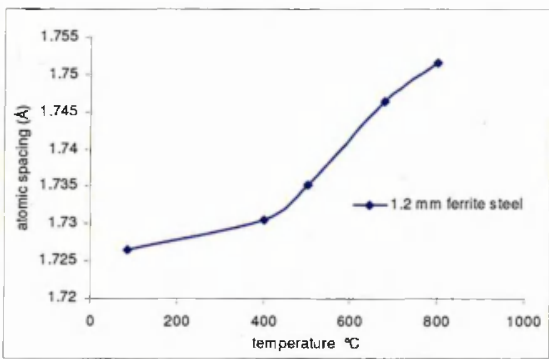

(d)

Figure 1.1: Previous proof of concept for the imaging of ferrite and austenite phases in steel: (a) diffraction image of $2 \mathrm{~mm}$ mild steel, (b) diffraction image of $2 \mathrm{~mm}$ stainless steel, (c) effects of temperature on the integrated diffraction profile of mild steel, (d) associated changes in atomic spacing.

explored as a possible technique to measure the phase transformation in hot rolled steel; the potential application being an online system in a working steel mill. Although the main focus was on the differences and thus the ability to distinguish between ferrite and austenite-phase sheet steel, a study was also carried out on temperature effects on the diffraction profiles approaching the phase transformation temperature. This effort demonstrated the ability to measure small changes in the inter-atomic spacings in a thick sample 
despite the associated attenuation effects (refer to Fig. 1.1) and provides the impetus for the current work. Given the demonstrated ability of the technique to distinguish between the primary ferrite and austenite phases, the technique is further developed in this work in order to detect the structural changes produced from the formation of the metastable martensite phase (an important avenue of research with regards to hardening steels used in the NNPP), and the lattice spacing changes associated with applied stress.

The remainder of this thesis is composed as follows:

- In Chapter 2 consideration is made of the fundamentals of X-ray scattering, from the classical relationship derived by Thomson, to the coherent (Rayleigh) scattering relationship, and finally to Bragg diffraction.

- Chapter 3 describes the experimental configurations and apparatus used, including X-ray tubes and semiconductor radiation detectors.

- In Chapter 4, preliminary work on low density samples, used to set up the apparatus is introduced. Work on low-alloy ferritic, and austenitic stainless steel is then presented, including both static and dynamic stress measurements on 304L stainless steel. Investigation through the use of transmission XRD, of martensite formation in cryogenically cooled austenitic stainless steel is also presented in depth. Finally, high energy diffraction measurements are discussed.

- Chapter 5 briefly discusses possible future developments, both of equipment and possible new measurements.

- Chapter 6 concludes the discussion. 


\section{Chapter 2}

\section{Elastic Scattering of X-rays}

\subsection{Introduction}

The founding theoretical work for elastic X-ray scattering was first produced by Thomson in 1906 [2]. Based upon classical electrodynamics, essentially an incoming electromaǵnetic wave causes a free electron to accelerate, producing an oscillatory motion as the wave passes. Given that an accelerating charge will radiate, this produces a second wave of the same frequency and phase as the incoming wave, 'scattering' of the wave is therefore the result.

Thomson scattering is however an idealised model for elastic scattering and does not extend to multiple bound electrons for the simple reason that it does not consider the effects of interference between the scattered waves from different electrons of the same atom.

Although his name is synonymous with the scattering of X-rays from atoms, the work of Lord Rayleigh (John William Strutt) on scattering concerned the scatter of visible light in the atmosphere which culminated in his 
1871 publication [15]. The early theoretical basis for Rayleigh scattering of X-rays was produced during the 1920s and 30s [16][17][18]. In these models Rayleigh scattering is described by a modified form of the relationship proposed by Thomson, accounting for the effect that the electronic charge distribution within an atom has on the total scattering.

While the Thomson scattering relationship is originally a classically derived expression from conventional electrodynamics (a quantum mechanical treatment in the low energy limit yields the same result [19]), Rayleigh scattering is based upon a quantum mechanically derived expression for the electronic charge distribution and, for heavier atoms, incorporates the effects of relativistic electrons in the inner atomic orbits.

The discovery of $\mathrm{X}$-ray diffraction was a result of the pioneering work of Laue (1912, Nobel prize 1914) [20][21] and later W.H Bragg and his son W.L. Bragg (1913, Nobel prize 1915) [22]. Diffraction is a well understood classical phenomenon dating back to Young's double slit experiment. With regards to the work of Bragg, it is the result of interference between X-rays scattered from a crystalline structure. This discovery allowed for the development of a myriad of applications where knowledge of the structure and orientation of a crystal is required, perhaps the most widely used example of this being in the field of protein crystallography.

\subsection{Thomson scatter from a free electron}

The basis for the derivation of the differential Thomson scatter cross section $\frac{d \sigma_{T}}{d \Omega}$ is firmly rooted in classical electrodynamics and the property that ac- 
celeration of a point-like charge will produce an electromagnetic wave [23] where:

$$
\frac{d \sigma_{T}}{d \Omega}=\frac{r_{0}^{2}}{2}\left(1+\cos ^{2} \theta\right)
$$

Noting that $r_{0}$ is the classical electron radius given by $r_{0}=\frac{1}{4 \pi \epsilon_{0}} \frac{e^{2}}{m c^{2}}$

The total Thomson scattering cross section $\sigma_{T}$ may be obtained from simple consideration of the power radiated by an accelerating charge, and the incident energy from the incoming electromagnetic wave in the $v \ll c$ limit. Consider a plane wave incident on a free electron where the instantaneous values of the electric and magnetic fields are given by:

$$
\begin{aligned}
& \boldsymbol{E}=E_{0} \sin (\omega t) \hat{\mathbf{i}} \\
& \boldsymbol{B}=\frac{E_{0}}{c} \sin (\omega t) \hat{\mathbf{j}}
\end{aligned}
$$

The energy incident upon the electron is given by the Poynting Vector [24], essentially the power flow per unit volume in the direction $\hat{\mathbf{k}}$ :

$$
S=\frac{\boldsymbol{E} \times \boldsymbol{B}}{\mu_{0}} \hat{\mathrm{k}}
$$

Therefore from 2.2 :

$$
\boldsymbol{S}=\frac{E_{0}{ }^{2}}{\mu_{0} c} \sin ^{2}(\omega t) \hat{\mathbf{k}}
$$

The energy per unit time radiated by the electron is given by the Larmor formula [24] in the $v \ll c$ limit, which shall not be derived here: 

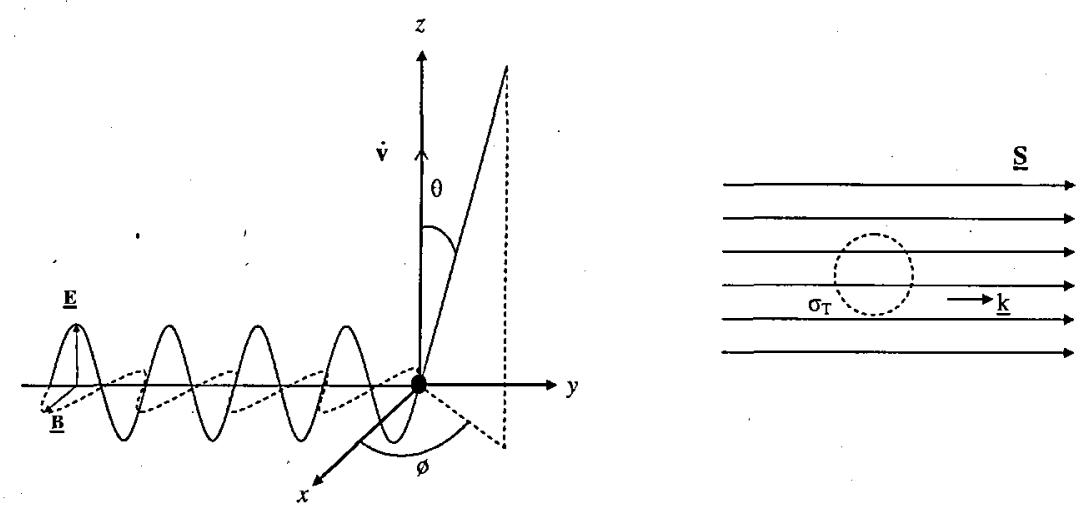

Figure 2.1: The variables involved in deriving the Thomson scatter cross section. The electric component of the incident EM wave produces an acceleration $\dot{v}$, of the electron. The power flow is represented by the Poynting vector $S$, the area through which it deposits energy to the electron represented by $\sigma_{T}$.

$$
\frac{d W}{d t}=\frac{e^{2} \dot{v}^{2}}{6 \pi \epsilon_{0} c^{3}}
$$

Where $\dot{v}$ is the acceleration of the electron. If $v \ll c$, the acceleration may be simply derived classically from $F=m a$, and the Larmor expression may be written in terms of $E, e$ and the electron mass $m_{e}$ :

$$
\frac{d W}{d t}=\frac{e^{4} E^{2}}{6 \pi \epsilon_{0} c^{3} m_{e}^{2}}
$$

Referring to Fig. 2.1 the total cross section represents the area through which the Poynting vector is flowing and depositing energy to the electron. The fraction of the total power is therefore simply the product of $S$ and $\sigma_{T}$ (note: the differential cross section $\frac{d \sigma_{T}}{d \Omega}$, is defined as the energy radiated per unit 
time per unit solid angle divided by the incident energy per unit time per unit area). Thus using equations 2.4 , and 2.6:

$$
\frac{E_{0}^{2}}{\mu_{0} c} \sin ^{2}(\omega t) \sigma_{T}=\frac{e^{4}}{6 \pi \epsilon_{0} c^{3} m_{e}^{2}} E_{0}^{2} \sin ^{2}(\omega t)
$$

Knowing that for electromagnetic waves, $v$, and therefore $c$, is equal to $\frac{1}{\sqrt{\mu_{0} \epsilon_{0}}}$ yields the final result for the total scatter cross section:

$$
\sigma_{T}=\frac{e^{4}}{6 \pi \epsilon_{0}^{2} c^{4} m_{e}^{2}}
$$

Which is independent of the incident wave frequency and has a value of 0.665 barns $\left(1\right.$ barn $=10^{-24} \mathrm{~cm}^{2}$ ).

\subsection{Rayleigh scatter from bound electrons}

When considering scattering from bound atomic electrons, the total scattering amplitude cannot be considered as simply a function of $Z$, i.e. summing the Thomson relationship over $Z$ electrons for that atom. Rather, in a multiple electron atom, the resultant scattered wave is produced from the combined scattering of all atomic electrons and more importantly, the interference effects that take place between the individual scattered waves. This interference is angular dependant; in the forward $\left(0^{\circ}\right)$ direction all scattered waves will be in phase thus constructively interfering. At increasing scattering angles the phase differences result in increasing destructive interference and the contribution of elastically scattered X-rays becomes negligible when compared to that of incoherent (Compton) scattering. 


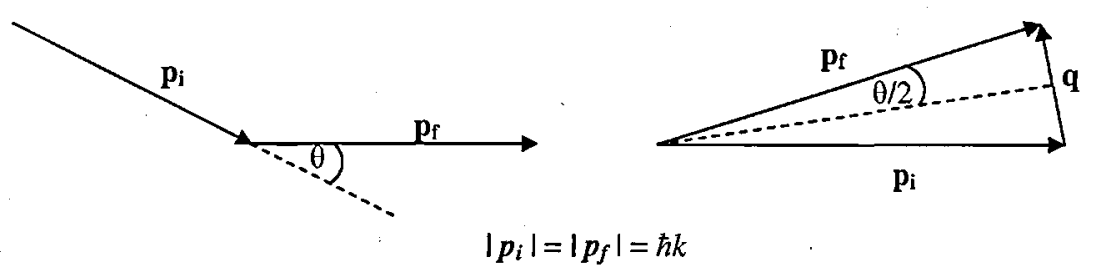

Figure 2.2: Derivation of the momentum transfer for an elastically scattered photon. An incident photon is scattered through a total angle of $\theta$. The momentum transfer $q$ may be obtained by considering that the modulus of the initial and final momentum is the same (noting that the recoil momentum of the atom is negligible).

It is important to now recall that X-rays are not simply waves, and to consider the discrete, photon aspect of electromagnetic radiation. The resultant scattering from an atom of multiple bound electrons is considered as a function of both $Z$ and the parameter $q$, the momentum transferred to the photon to scatter it through the angle $\theta$. Referring to Fig. 2.2. and considering a photon of initial momentum $p_{i}$, scattered through a total angle of $\theta$; it can be seen that for elastically scattered photons the momentum transferred is given by:

$$
q=2 \hbar k \sin \left(\frac{\theta}{2}\right)
$$

Where it is the angle $\frac{\theta}{2}$ that is referred to as the 'glancing angle'.

The function of $q$ and $Z$ that describes the scattering from bound electrons is known as the atomic form factor. In the form factor approximation, the Thomson scattering relationship is modified with the factor $[F(q, Z)]$ such that: 


$$
\frac{d \sigma_{R}}{d \Omega}=\frac{d \sigma_{T}}{d \Omega}[F(q, Z)]^{2}
$$

As demonstrated in section 2.2, the total Thomson scatter cross section $\sigma_{T}$ is simply a constant of 0.665 barns. The form factor is therefore proportional to the square root of the scatter intensity, in effect representing the ratio of scattered amplitude by the atom, to that of a single free electron.

While the Thomson scatter cross section is a classically derived expression, the calculation of the form factor lies with solution of the Schrödinger equation for systems of many fermions. Since it is not feasible to solve the Schrödinger equation directly for atoms beyond helium, it (and thus the form factor) must be approximated.

One of the simplest bases for the calculation of atomic form factors is the Hartree method and the one electron approximation [16][25]. Each electron is represented by its own wavefunction, effectively reducing the problem to that of a single electron, moving in the field of the nucleus in addition to a field that represents the average effect of all the other electrons. The method is then essentially the variational principle, with the trial wavefunction being the product of multiple one-electron wavefunctions.

It is sufficient to state here that for a spherically symmetric charge density. $\rho(r)$ the form factor is calculated from:

$$
F[q, Z]=4 \pi \int_{0}^{\infty} \frac{\rho(r) \sin (q r) r^{2} d r}{q r}
$$

This technique of calculating the form factor is also extended to heavier atoms 
with relativistic inner electrons. The form factor in this case is modified such that for the $i$ th subshell the form factor is given by:

$$
g_{i}(q)=4 \pi \int_{0}^{\infty} \frac{\rho(r) \sin (q r)}{q r}\left[\frac{m_{0} c^{2}}{E_{i}-V(r)}\right] r^{2} d r
$$

Where $E_{i}$ is the total energy of the $i t h$ electron, $V(r)$ is the potential of the electronic charge at a radial distance $r$. The total form factor is thus obtained by summing $g_{i}$ over all electron subshells.

As discussed previously, the simplest methods for calculating the atomic form factor are based upon the Hartree method. Overall this is a very good approximation, however it begins to break down for very low/very high energies. Similarly the approximation does not hold when photon energies are comparable to the $\mathrm{K}$-shell binding energies of the scattering material. In order to obtain more accurate results for these regimes a complete treatment based upon quantum electrodynamics is required. Further discussion of these methods of deriving the atomic form factors is available in the work of Kane et al [26].

Extensive effort has been expended on tabulating atomic form factors over the last 30 years and these are now collated for all the elements in the region $1 \leq Z \leq 92$ in the National Institute of Standards and Technology (NIST) Physics laboratory database [27]. A review of measurements of the total elastic scattering cross-sections in the range $1 \mathrm{keV}$ to $4 \mathrm{MeV}$ is covered in the work of Bradley et al [28]. 


\subsection{Bragg diffraction}

The elastic scattering considered thus far has concerned the interference of scattered waves from electrons within the same atom. Longer range interference effects take place between scattered waves from the electrons of neighbouring atoms. This effect is noticeable in the diffraction image produced from scattering of X-rays off an amorphous material such as water. In this example a broad feature diffraction pattern is produced as a result of interference between X-rays scattered from neighbouring water molecules.

The most striking diffraction patterns occur for substances where atoms are arranged in a regular periodic array. In such a material atoms are arranged in regular layers, where the spacing between layers is $d$. Constructive interference between atoms in the different layers gives rise to the phenomenon of well defined diffraction fringes as discovered by W. H. and W. L. Bragg in 1913.

Referring to Fig. 2.3, incident X-rays of wavelength $\lambda$ are scattered through a total angle $\theta$ by identical atoms in layers $A$ and $B$. Through consideration of the geometry it is clear that the path difference $(\mathrm{P})$ between the scattered waves is given by:

$$
P=2 d \sin \left(\frac{\theta}{2}\right)
$$

For constructive interference to occur the scattered waves must be in phase, thus the path difference must equal an integer number of wavelengths. This leads to the Bragg condition where: 


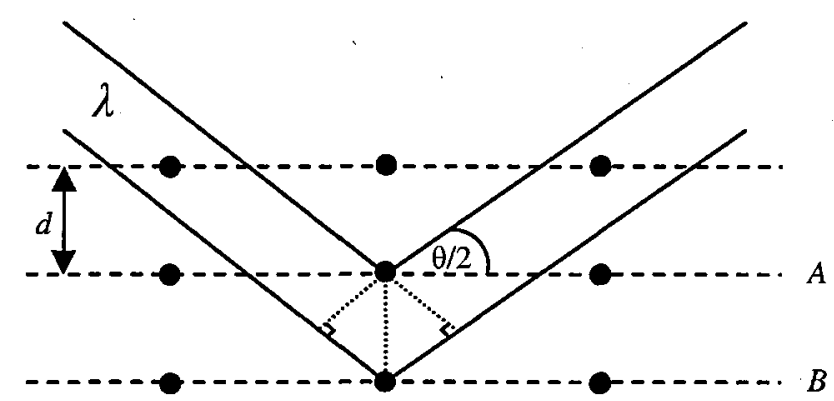

Figure 2.3: Geometric considerations for deriving the Bragg equation. Considering two planes in a regular crystal structure it is clear that, for similar X-rays elastically scattered off atoms in neighbouring planes, a path difference will occur as a function of scattering angle and the separation of the planes.

$$
n \lambda=2 d \sin \left(\frac{\theta}{2}\right)
$$

Therefore given that there are multiple planes in a crystal, a series of peaks at different scattering angles can be produced. In the simplest example of the powder diffraction technique, the wavelength is fixed through monochromation and the scattering profile over all scattering angles is measured, producing a series of diffraction rings onto photographic film.

It should be noted that the relative intensities of the diffraction lines are more complicated. The intensity of a given line ' $k$ ' is a function of the scattering by all atoms. In section 2.3 the scattering from a single atom is described by the atomic form factor $F[q, Z]$. When considering a crystal structure, the scattering is the sum of that for all atoms in a unit cell (the repeating unit that forms the crystal lattice) while taking into account the interference of the scattered waves (i.e. phase). The result of these considerations is the 


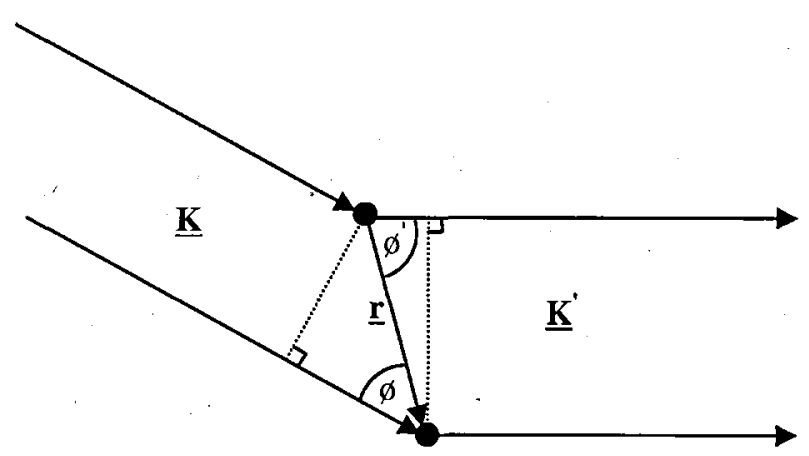

Figure 2.4: Derivation of the phase factor for Bragg diffraction. 'The phase factor arises from consideration of the path difference between parallel $\mathrm{X}$-rays and the requirement of an integer number of wavelengths for constructive interference.

'structure factor' $F_{s f}$ which is derived below.

Firstly the interference of the waves must be considered. Referring to Fig. 2.4; for an incident wave of wavevector $\boldsymbol{K}$, re-radiating with wavevector $\boldsymbol{K}^{\prime}$, the associated path difference obtained from consideration of the geometry is given by:

$$
P=|\boldsymbol{r}| \cos (\phi)-|\boldsymbol{r}| \cos \left(\phi^{\prime}\right)
$$

Therefore considering the requirement of a whole number of wavelengths for constructive interference this may be written as:

$$
n \lambda=\frac{\boldsymbol{r} \cdot \boldsymbol{K}}{|\boldsymbol{K}|}-\frac{\boldsymbol{r} \cdot \boldsymbol{K}^{\prime}}{\left|\boldsymbol{K}^{\prime}\right|}
$$

For elastic scattering $|\boldsymbol{K}|=\left|\boldsymbol{K}^{\prime}\right|$ where $|\boldsymbol{K}|=\frac{2 \pi}{\lambda}$, therefore yielding the phase factor: 


$$
2 \pi n=r \cdot(\Delta K)
$$

Combining the sum of the atomic form factors $f_{n}$ with the phase factor derived above, yields the final result for the structure factor, and thus the total scattering amplitude from a unit cell of $n$ atoms:

$$
F_{s f}=\sum_{1}^{n} f_{n} e^{(i r \cdot \Delta K)}
$$

The intensity of the diffraction lines is therefore proportional to $\left|F_{s f}\right|^{2}$. There are however, other considerations such that the intensity for a given diffraction line is:

$$
I_{k}=\left|F_{s f}\right|^{2} F_{m} F_{L p}
$$

Where $F_{m}$ is the multiplicity factor and $F_{L p}$ is the Lorentz polarisation factor. The multiplicity factor takes into account different planes diffracting at the same angle, thus increasing the scatter intensity at that angle. It is therefore simply an integer representing the number of equivalent sets of planes.

The Lorentz-polarisation factor is a combination of factors accounting for both an unpolarised incident beam (therefore containing electric field components in both the $y$ and $z$ directions), and consideration of the geometric arrangement of the scattering sample with respect to the incident and diffracted beams. The combined factor is wholly angular dependant and given by: 


$$
F_{L p}=\left(\frac{1+\cos ^{2}(\theta)}{\sin ^{2}\left(\frac{\theta}{2}\right) \cos \left(\frac{\theta}{2}\right)}\right)
$$

Overall therefore, the simplicity of the Bragg condition given in 2.14 belies the complexity of the actual diffraction pattern produced.

X-ray diffraction has now been established for over 90 years as an experimental technique. As such, the complete diffraction patterns of many materials are well known. An extensive, global library of data concerning almost half a million crystalline materials is available, maintained by the International Centre for Diffraction Data(ICDD) [29]. 


\section{Chapter 3}

\section{Experimental configurations and apparatus}

\subsection{Introduction}

The serendipitous discovery of X-rays was as a result of the work of Röentgen in late 1895. While investigating the range of cathode rays (thermionically emitted electrons) generated from a Crookes tube, Röentgen noticed that a barium platinocyanide-coated screen some distance away began to glow. Given that his apparatus was screened with cardboard he deduced this could not be as a result of cathode rays, and while investigating this new phenomena with various screening materials, he accidentally produced an image of the bones within his own hand. Further images, including the famous radiograph of Mrs Röentgen's hand presented in his paper of December that year, earned Röentgen the first Nobel prize for physics in 1901.

Generation of X-rays for coherent scattering experiments can be per- 
formed in a number of ways. Low intensities of monochromatic X-rays are generated from radioactive sources, either indirectly through ionising interactions producing vacant orbitals that are filled by de-excitation of electrons from higher orbitals, or directly through the processes of internal conversion and electron capture. Internal conversion is a competing process to the usual de-excitation of the nucleus through emission of a gamma ray [30], the unstable nucleus transfers sufficient energy to one of the inner shell electrons to allow it to escape the atom. The sharp X-ray lines generated from a source as a result are due to the subsequent de-excitation of electrons in higher shells to fill this vacancy.

While a monochromatic source is desirable for some measurements, a polychromatic source is often required. After 110 years, thermally generated electrons accelerated into a target still provide the basis for most X-ray work; X-ray tubes are being continually developed for uses from radiography in hospitals and industrial processes such as weld inspection, to security applications such as those described in chapter 1.

For the generation of higher intensity polychromatic and monochromatic $\mathrm{X}$-rays electron accelerators are required. In the latest generation of synchrotrons electrons are accelerated up to energies of several $\mathrm{GeV}$ before being passed through a series of bending magnets ('undulators' and 'wigglers') to produce various energies of X-rays. Such facilities are capable of generating beams 11 or 12 orders of magnitude more brilliant (photons $/ \mathrm{s} / \mathrm{mm}^{2} / \mathrm{mrad}^{2} / 0.1 \%$ ) [31] than a typical laboratory X-ray tube. It should however be noted that the start-up costs of this generation of synchrotrons (for example PETRA III in Germany) are now of the order $£ 100$ million [32]. 
For the detection of coherently scattered X-rays a number of solutions exist dependant on the intended application and the experiment configuration.

When employing polychromatic X-rays in the application of measuring oil/water ratios mentioned in chapter 1 ; the requirement was for a robust, energy resolving detector (given a polychromatic source was employed) with relaxed constraints on the energy resolution. In this case CdZnTe detectors were employed giving suitable energy resolution without the need for expensive cryogenic cooling systems.

In the case where monochromatic $\mathrm{X}$-rays are used and where simultaneous measurement of scattered X-rays over all angles is the objective, the simplest form of detector is a photographic plate, directly recording the diffraction image. Further development of this technique involves the use of a CCD camera coupled to an image intensifier, allowing the construction of an integrated diffraction profile of the scattered X-rays based upon the position (i.e. scatter angle) and the intensity of the scattered X-rays. Detector systems (including the specifics of the devices used in this work) and new developments in detectors applicable to coherent X-ray scattering are discussed in section 3.4 .

\subsection{Experiment configurations}

Referring to the momentum transfer $q$ derived in section 2.3 it is clear that the resultant scattering, being a function of $q$, will therefore be a function of photon energy and the angle $\frac{\theta}{2}$. 


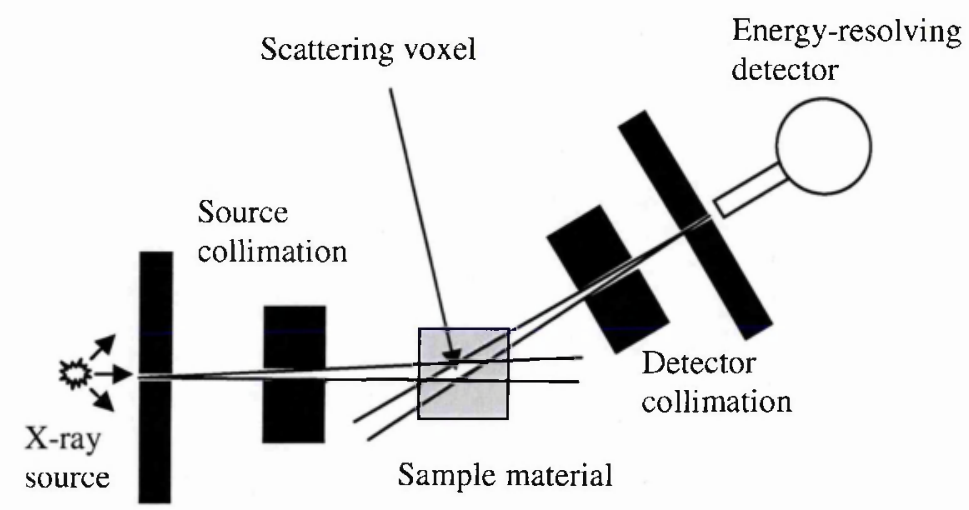

Figure 3.1: Schematic of a typical energy dispersive configuration. Using a series of simple collimators the spatial resolution of the beam is well defined such that a small volume or 'voxel' of the material may be sampled. This voxel is a trapezoid defined by both the source and detector collimators.

This gives rise to two possible experiment configurations which are illustrated in Fig. 3.1 and 3.2; energy dispersive where the incident photon energy is varied and the apparatus set at a fixed angle $\frac{\theta}{2}$, or angular dispersive where a monochromatic source is used while varying the angle $\frac{\theta}{2}$.

In practice the energy dispersive technique relies on a polychromatic Xray source, where multiple energy photons are scattered by the sample of interest simultaneously and discriminated by an energy resolving detector. The region sampled by this technique, the 'scattering voxel', is defined by the geometry of the source and detector collimators as depicted in Fig. 3.1.

In an angular dispersive technique, for example such as that used in [14], elastic scattering at all angles is recorded simultaneously for a single fixed energy. This does not require an energy resolving detector but rather a position sensitive system to record the diffraction image produced. By 
utilising a CCD coupled to an image intensifier the resulting diffraction image (e.g. refer to Fig. 1.1) may be used to construct an integrated profile i.e. a series of peaks corresponding to constructive interference between scattered waves and therefore structure of the material of interest.

For both techniques the resulting scattering spectrum can be calibrated, not in terms of the energy of the scattered photons, or the angle through which they are scattered, but instead the parameter $x$ where:

$$
x=\frac{1}{\lambda} \sin \left(\frac{\theta}{2}\right)
$$

Thus relating the resultant spectrum to the momentum transfer $q$ (referring to Eqn. 2.9 it is clear $x \propto q$ ). Both techniques therefore achieve the same result; sampling of the elastic scatter cross section in momentum space. Calibrating in terms of $x$ is further useful in that the inter atomic spacings in crystalline structures $d$ may then be obtained. Comparing equation 3.1 to the Bragg equation it is clear the two are related by the relationship $x=\frac{1}{2 d}$ (for $n=1$ ).

Although both techniques essentially arrive at the same result (i.e. a measurement of the elastic scatter cross section in momentum space), there are a number of advantages and disadvantages to each that must be considered when determining which technique is suitable for a given application.

In energy dispersive techniques the full range of energies generated by the $\mathrm{X}$-ray source is utilised. This has the advantage of shorter measurement times required to build up sufficient statistics for the resulting spectrum. The apparatus required is also relatively simple in that the data is collected at a single 


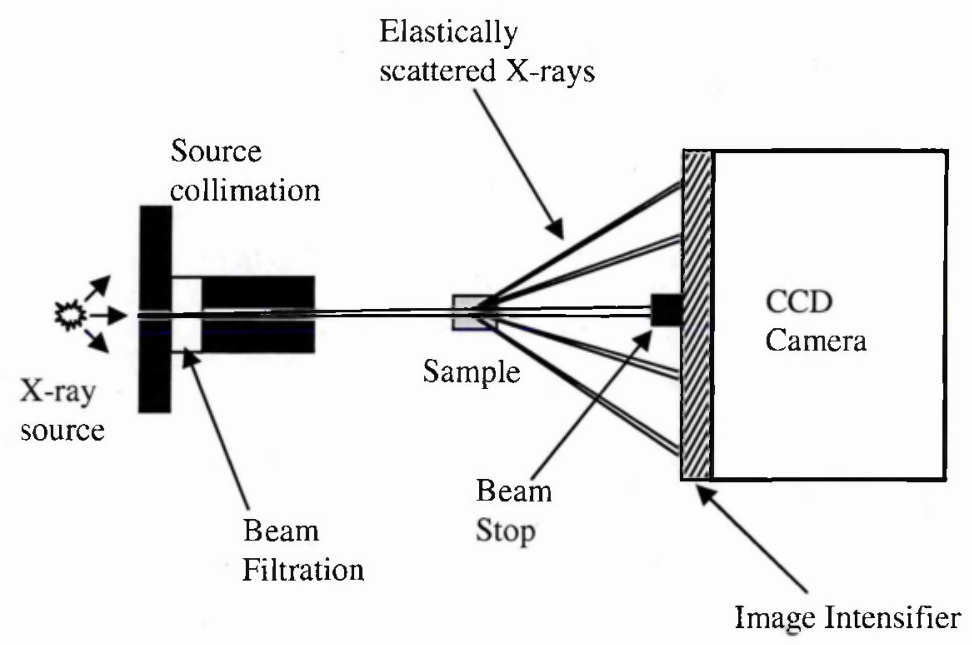

Figure 3.2: Fxample of an angular dispersive configuration. Mono-energetic $\mathrm{X}$-rays are scattered elastically from the target sample at multiple angles simultaneously, resulting in the production of the diffraction 'rings' as seen in Fig. 1.1. The beam stop must be carefully positioned to cut out the direct transmitted beam (that would otherwise saturate the image) without removing information from photons scattered through smaller angles.

angle, allowing for a fixed system consisting of source, collirnator and detector. A disadvantage to this technique lies with the problem of differential attenuation in thicker samples. Because a range of energies is used, the effect of lower energy X-ray photons being preferentially attenuated by scattering and photoelectric absorption will be noticeable. This effect, demonstrated in Fig. 3.3 would be noticed as a change in the relative intensities of peaks in the resulting spectrum; the low angle peaks corresponding to higher energy photons becoming more pronounced relative to the higher angle (low energy) peaks. It should be noted that the effect may be mitigated by looking at the $0^{\circ}$ angle and normalising the scatter spectrum to the transmitted beam. 


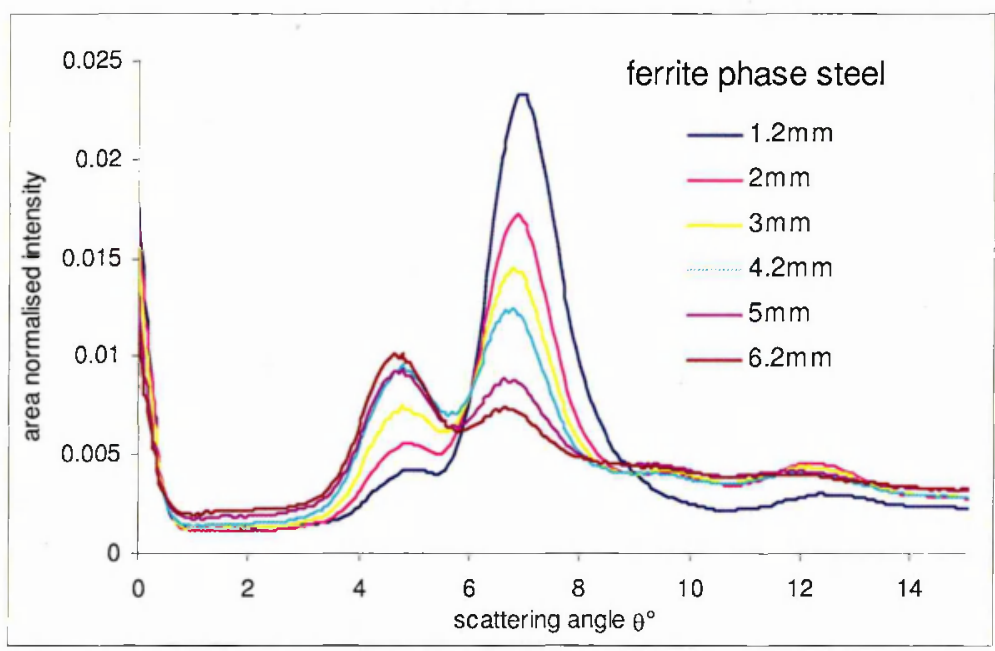

Figure 3.3: Example of the differential attenuation effects in increasing thicknesses of mild steel caused by the attenuation of low energy photons [14].

Angular dispersive techniques, utilising a fixed incident energy do not encounter this problem. The difficulty in generating a monochromatic beam of sufficient intensity does however present a problem. Quasi-monochromatic beams may be generated by suitable filtering of polychromatic beams so that only well defined characteristic X-rays (corresponding to removal of an electron) are used. This relies on selecting a filtering material with a suitable 'K-edge' in its absorption cross-section. The K-edge is a discontinuity in the absorption spectrum, corresponding to the energy of photoelectric absorption for a K-shell electron. By selecting a filter material with a K-edge just below the desired X-ray wavelength (refer to Fig. 3.4), unwanted lower-energy Xrays may be removed from the beam [33]. The efficiency of the filter depends on the thickness, an increase of which will lead to a cleaner single energy beam. There is however, a compromise to be struck between producing a 


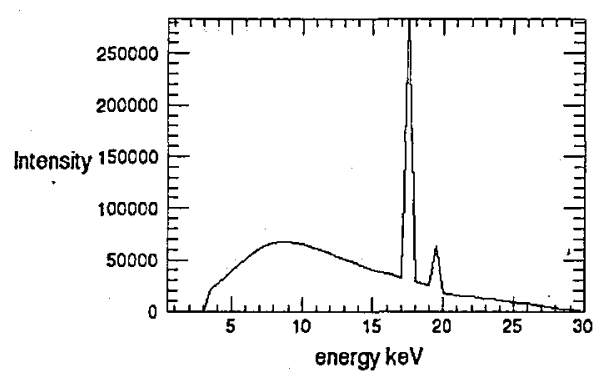

(a)

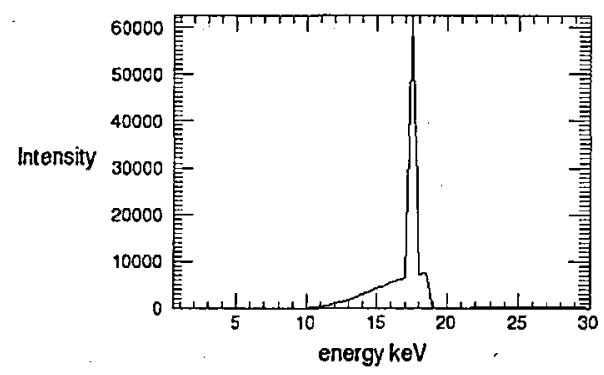

(b)

Figure 3.4: Illustration of the application of a suitable filter to remove unwanted $\mathrm{X}$-rays and thus produce a quasi-monochromatic beam. Here the X-rays are those of Molybedenum, the filter applied in this case is Niobium (simulated using the IPEM Report 78 Spectrum Processor software) [34].

sharply defined mono-energetic beam and maintaining a useful beam intensity. Because of this, a slight blurring effect resulting from multiple energies in the beam must be tolerated to maintain realistic measurement times.

\subsubsection{Energy dispersive apparatus}

Prior to October 2005, the highest power-rating X-ray tube available was a $80 \mathrm{kV} / 250 \mu \mathrm{A}$ tungsten target device (Source-ray, Inc: SB-80-250), limiting the ability to image higher density materials (for example steel) to thin samples. The work carried out with this tube has therefore been focused on low density samples, including but not limited to sugar and a polyviologen-silica hybrid (PVG/SiO 2$)$ nanopowder sample.

In addition to the $80 \mathrm{kV}$ tube (used for energy dispersive work), a $50 \mathrm{kV} / 1 \mathrm{~mA}$ molybdenum target tube (Oxford XTF5011) has been utilised, primarily for angular dispersive measurements with diffraction images collected using an 


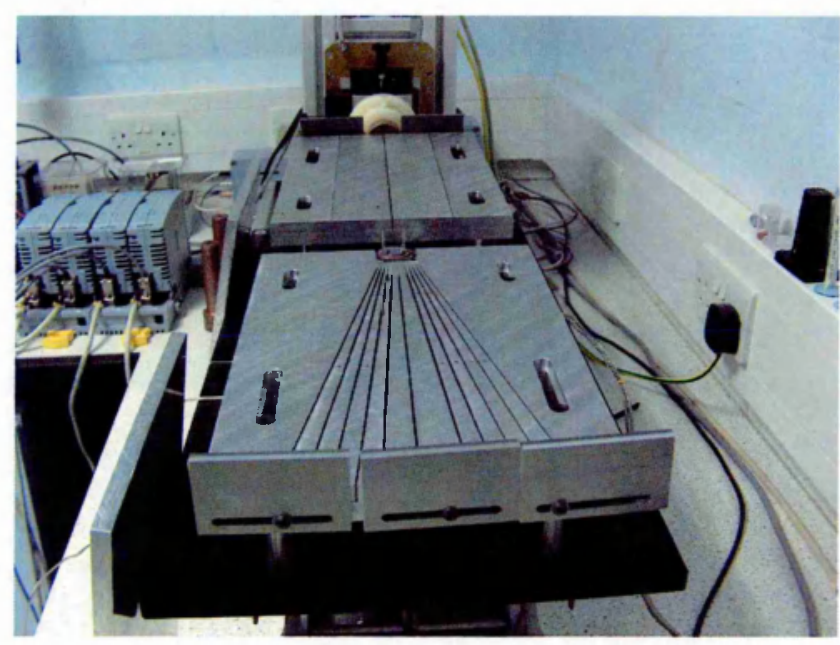

Figure 3.5: Photograph of the energy dispersive $\mathrm{X}$-ray diffraction apparatus used in late 2004/early 2005. The aluminium collimator rig shown here was loaned from City University (courtesy of M.J Farquharson) and has since been replaced by an identical apparatus. The $80 \mathrm{kV}$ X-ray tube is visible in the background with the cylindrical collimator mounted on $\mathrm{x}-\mathrm{y}$ micro-positioners.

X-ray image intensifier.

As discussed in section 3.2 energy dispersive measurements use the unfiltered bremsstrahlung spectrum (for which tungsten target tubes are particularly suitable) in conjunction with an energy-resolving detector. A Peltiercooled Amptek XR-100T CZT device (see section 3.4.2) was initially used for energy dispersive work involving low density samples, including for example, sugar.

The collimator system first used for energy dispersive measurements is shown in Fig. 3.5. A cylindrical copper-coated lead collimator forms the initial, coarse collimation of the source. The beam is then further collimated by 
a fixed, $30 \mathrm{~cm}$ aluminium block into a $1 \mathrm{~mm}$ by $10 \mathrm{~mm}$ tall ribbon beam. The advantages of this geometry over that of a pencil beam have been previously demonstrated [4], namely allowing for a higher incident flux while maintaining suitable angular resolution. The detector collimation is mounted on the same apparatus, consisting of an equally-sized $(30 \mathrm{~cm})$ aluminium block with $1 \mathrm{~mm}$ collimation provided for the transmitted $\left(0^{\circ}\right)$ beam and for fixed angles in $1^{\circ}$ degree steps between $3^{\circ}$ and $12^{\circ}$. Scattering is measured for only a single angle at a time, with screens consisting of $3 \mathrm{~mm}$ aluminium bonded to $3 \mathrm{~mm}$ lead drawn into place to block all other scattering angles.

Given that the coherent scattering signal is weak compared to the transmitted beam it is important to ensure the highest X-ray flux.possible is utilised. Micropositioners in both the $\mathrm{X}$ and $\mathrm{Y}$ planes were. used in conjunction with a NE Technology Limited Farmer dosimeter to allow for 'mapping' of the X-ray tube focal spot ( $\mathrm{a} 3 \mathrm{~mm}$ by $3 \mathrm{~mm}$ area), thus ensuring the collimator is placed over the region of highest $\mathrm{X}$-ray flux. In addition to positioning the primary collimator over the region of highest intensity, the aluminium collimator rig requires careful positioning to ensure the beam remains aligned across the complete rig. Coarse adjustment was achieved using a laser, while finer adjustment was performed using the dosimeter; i.e. using the known dose-rate at the exit of the cylindrical collimator, and comparing that to the dose-rate at the exit of the aluminium collimator (while accounting for effective cross sectional area of both collimators and $1 / r^{2}$ ) to achieve optimum alignment.

From February 2006 a 225kV Comet MXR225/22 X-ray tube was available for use in a dedicated X-ray laboratory at the University of Surrey. The 
primary aim for this apparatus was to image the change in lattice spacings in austenitic stainless steel samples due to, for example, applied stress.

The tungsten target tube provides an order of magnitude increase in flux over the X-ray tubes used previously and more importantly, sufficiently high energies of X-rays to penetrate several millimetres of steel. The complete experimental apparatus for energy dispersive XRD was updated and is shown in Fig. 3.6. In addition to the new X-ray tube, a new aluminium collimator apparatus was constructed (courtesy of HMS Sultan's workshop) and a highpurity, thin beryllium-windowed germanium detector (refer to section 3.4.2) replaces the CZT detector used previously. The Comet MXR225/22 X-ray tube is mounted inside a $4 \mathrm{~mm}$ lead-lined enclosure to reduce leakage from the tube itself. A $20 \mathrm{~mm}$ diameter exit port has been created to which various cylindrical collimators may be inserted, such that they are positioned directly over the output window of the X-ray tube. The apparatus for mounting the collimator system and germanium detector was constructed from BoschRexroth framework, allowing a significant degree of flexibility and reduction in weight and scatter compared to, for example, an all welded steel construction.

Given the increase in X-ray energy and flux over the previous experiments, increased source collimation has been implemented to ensure that only elastically. scattered X-rays from the sample are measured. A $10 \mathrm{~mm}$ diameter, cylindrical lead collimator is mounted inside the exit port of the $\mathrm{X}$-ray tube enclosure, providing a coarse pencil beam prior to the main source collimation. Initially this consisted of a series of coarse lead collimators, generating a $1 \mathrm{~mm}$ by $10 \mathrm{~mm}$ ribbon beam prior to the aluminium collimator 


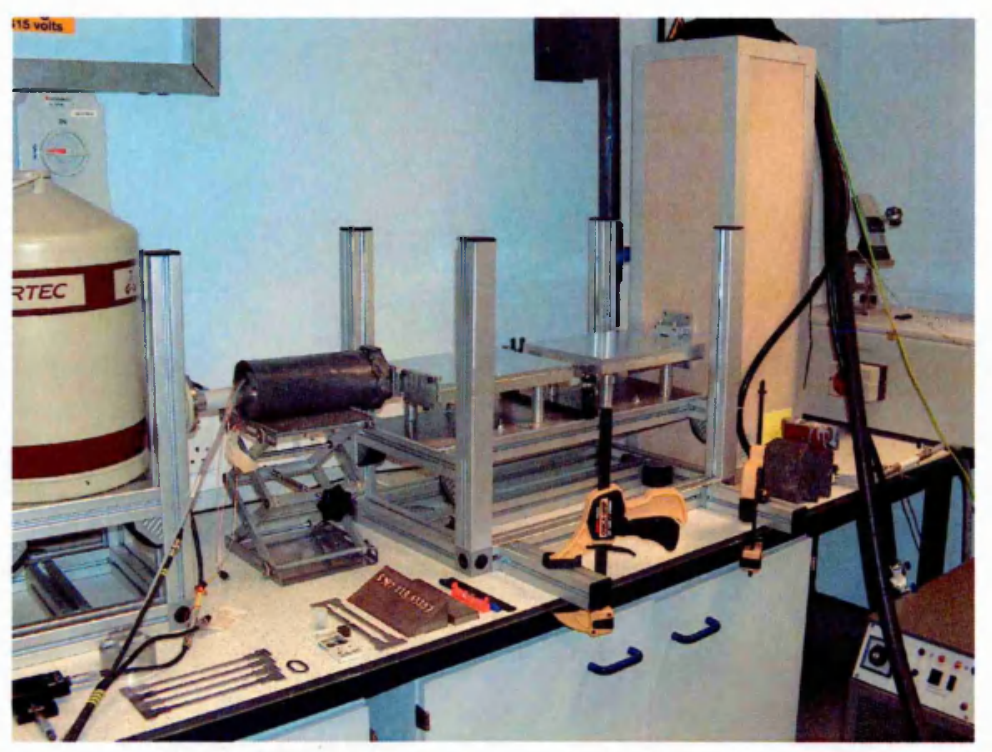

Figure 3.6: The updated apparatus used for energy dispersive XRD. A new $225 \mathrm{kV}$ X-ray tube (limited to $160 \mathrm{kV}$ ) is mounted inside a $4 \mathrm{~mm}$ lead-lined enclosure (far right) to reduce leakage from the tube itself. Given the higher X-ray intensities in use, extra collimation (HUBER tungsten-bladed variable slits) is included to form the initial ribbon beam. Furthermore the HPGe detector (far left) is encased in $2 \mathrm{~mm}$ lead to reduce background scatter from undefined angles.

apparatus. These have since been replaced by a set of precision-engineered HUBER tungsten-bladed slits to provide more finely tuned collimation in the $\mathrm{x}-\mathrm{y}$ plane. Further to the overall increase in the source collimation, the detector itself is shielded with a $2 \mathrm{~mm}$ thick lead sheath in order to reduce the extraneous scatter background. Elastically scattered X-rays from the sample at the defined angle of interest are allowed onto the detector window via a $1 \mathrm{~mm}$ by $10 \mathrm{~mm}$ vertical slit in the front face of the sheath. 


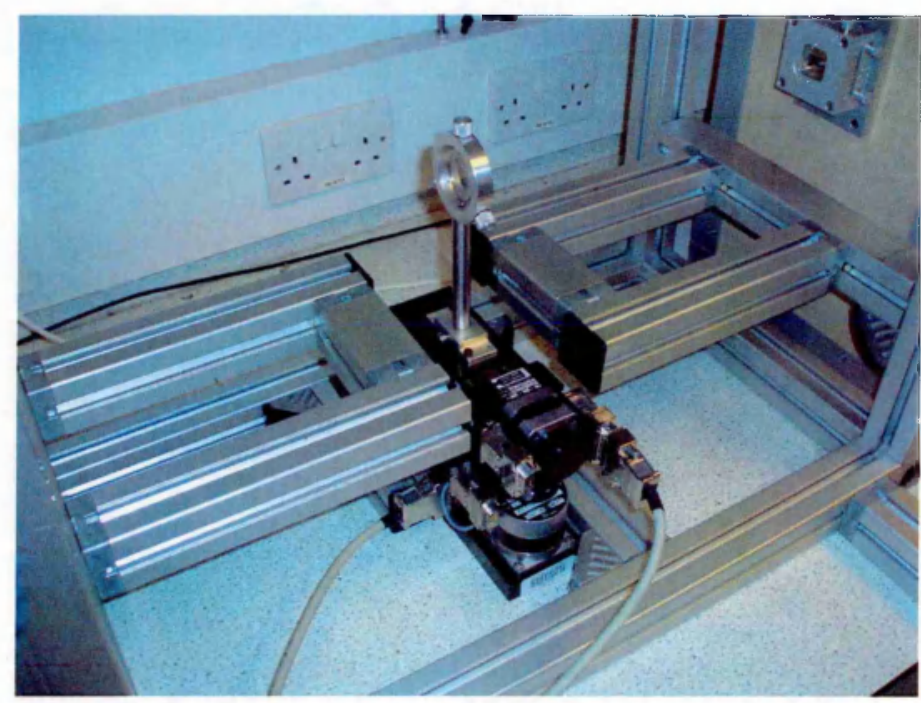

Figure 3.7: The two-direction stepper motor system used to produce line scans across the sample. The stepper-motors are mounted together below the collimator (removed for photographic purposes), while an aluminium optical post is fastened to the top, vertical stepper-motor, and passes through a hole cut in the base-plate of the collimator. At the top of the post a circular, 3-point clamp is used to securely fasten the sample in-place without interfering with the incident $\mathrm{X}$-ray beam.

The setup shown in Fig. 3.6 has been used for imaging ferrite and austenitephase steel samples up to several millimetres thick (refer to section 4.3.2). The system is however only capable of measuring single fixed points along the sample, with the scattering voxel fixed by the optical filter mounts used to clamp the sample in place. In order to incorporate the ability to produce a 2D map of a sample a stepper motor system was later constructed, allowing remote positioning of the sample relative to the fixed incident $\mathrm{X}$-ray beam. This system is shown photographically in Fig. 3.7 and has been designed 


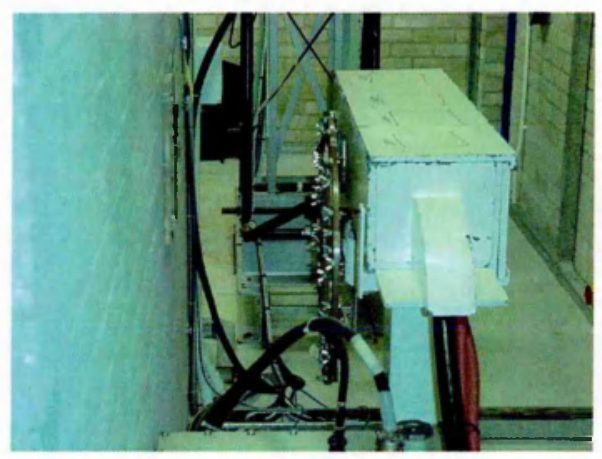

(a)

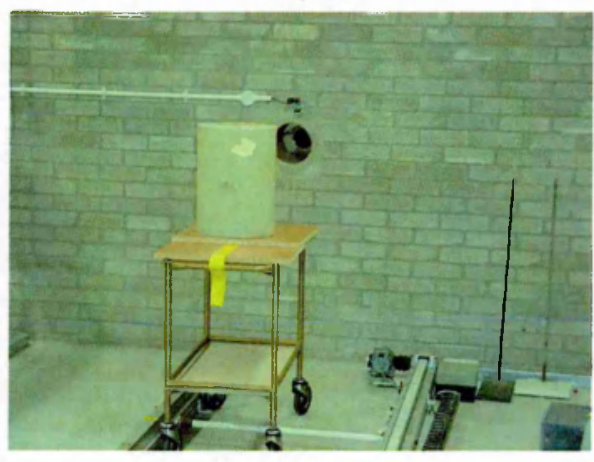

(b)

Figure 3.8: HMS Sultan's X-ray lab prior to the construction of the X-ray diffraction apparatus included an obstructing high-density concrete wall positioned in front of the X-ray tube. In (a) an X-ray filter mount can be seen which was removed at the same time as the wall. The wall is shown in (b), with an aperture $18 \mathrm{~cm}$ in diameter; too large to provide useful beam collimation, and too small to fit the aluminium collimator apparatus inside.

specifically for a series of oval and near-circular 304L stainless steel samples provided by HMS Sultan. As discussed further in section 4.3.2, these samples are used to investigate the martensite phase transformation upon cryogenic cooling in this particular alloy. Again the apparatus was constructed from Bosch-Rexroth framework, allowing the different experiment configurations to be easily interchanged.

In parallel to the development of a table-top system at the University of Surrey, development of a transmission X-ray diffraction system has taken place at HMS Sultan's Nuclear department. This system exploits an existing $225 \mathrm{kV}$ tungsten $\mathrm{X}$-ray tube, which early in the project was demonstrated to be in suitable working order for transmission diffraction experiments [35]. 


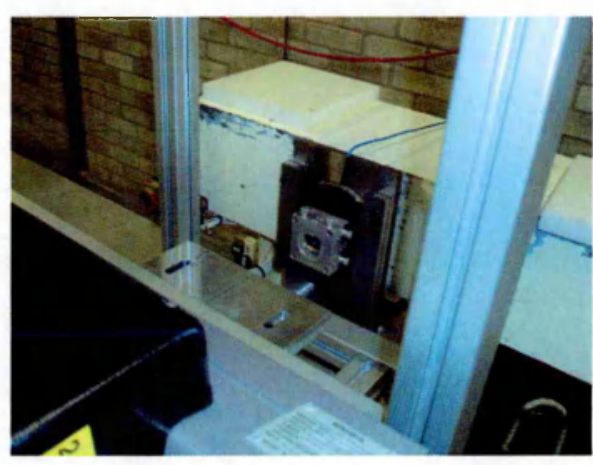

(a)

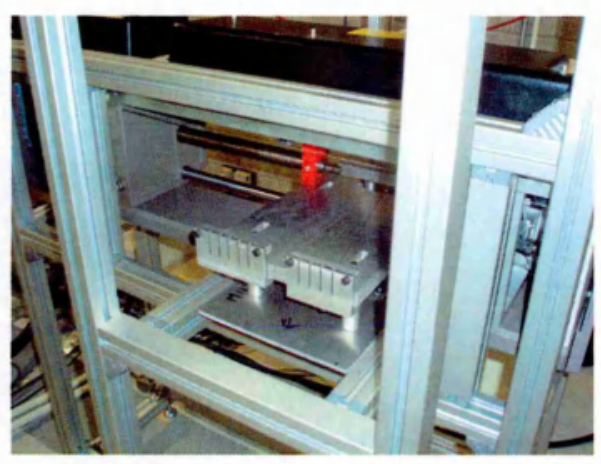

(b)

Figure 3.9: The initial apparatus was constructed at HMS Sultan given the availability of the tensometer and $225 \mathrm{kV} \mathrm{X}$-ray tube at the facility. (a) the updated collimator system on the $225 \mathrm{kV}$ X-ray tube is visible; consisting of a $1.73 \mathrm{~cm}$ diameter permanently mounted lead collimator and a removable HUBER 3013 tungsten-bladed slit screen. The detector mounted behind the slit is part of the safety interlock system. (b) the configuration of the Monsanto tensometer and pre-existing aluminium collimator apparatus.

The main experimental work to be carried out involved dynamic stress measurements of 304L austenitic stainless steel, measuring lattice spacing changes with elastic deformation. To this end a Monsanto tensometer was incorporated into the diffraction system, capable of applying up to a $20 \mathrm{kN}$ load to the samples. The complete apparatus is shown in Fig. 3.9, consisting of effectively two separate supporting structures (one for the collimator, one for the tensometer) built from Bosch-Rexroth aluminium framework. This design made for a very stable structure while allowing independent alignment of the collimator in the xyz planes, alignment of tensometer in the xyz planes and the correct alignment of the tensile-loaded sample with respect to the 


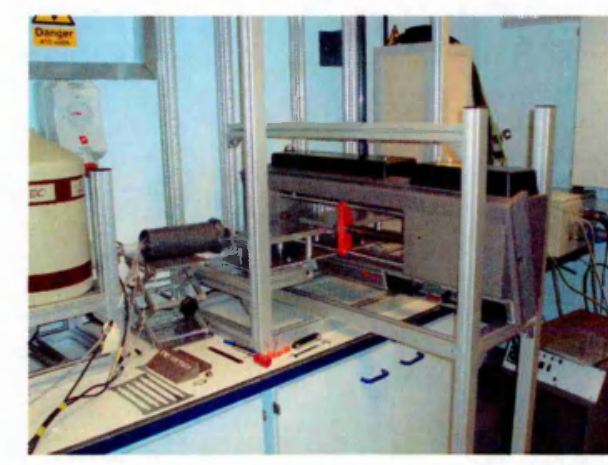

(a)

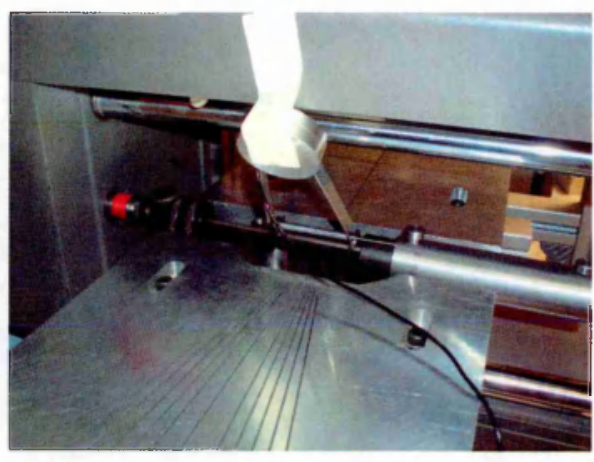

(b)

Figure 3.10: The dynamic stress apparatus as relocated to the Surrey X-ray lab. In (a) the configuration of the tensometer and collimator is shown with respect to the existing $\mathrm{X}$-ray tube and $\mathrm{HPGe}$ detector. In the initial experiment a strain gauge (as shown close up in (b)) was incorporated into the apparatus, however this was later shown to have a non-linear response, and for the remainder of the experiment measurements were made as a function of applied load in $\mathrm{kN}$.

incident X-ray beam.

Construction of this apparatus was delayed until November 2006, pending facility changes and the removal of an obstructing high density concrete wall positioned several tens of centimetres in front of the X-ray tube (refer to Fig. 3.8). This was initially designed to act as both a scattered radiation shield for an adjacent control room and a coarse collimator. However, in-house calculations demonstrated the wall was not in fact required to maintain dose rates below $1 \mu \mathrm{Sv} / \mathrm{hr}$ in the control room, and that the secondary function of collimator could be more effectively achieved using a $1.73 \mathrm{~cm}$ bore, lead collimator permanently mounted onto the existing lead-lined shielding containing the X-ray tube. 
Unfortunately soon after construction was completed, the laboratory at HMS Sultan had to be closed down due to an electrical fire. Since the timeframe for re-opening was unknown, the decision was taken to re-locate the apparatus to Surrey. The re-built apparatus is shown in Fig. 3.10 mounted in front of the Comet MXR225/22 X-ray tube. The beam geometry used is identical to that used for comparing austenite and ferrite-phase samples, i.e. a $1 \mathrm{~mm}$ by $10 \mathrm{~mm}$ ribbon beam with a $1 \mathrm{~mm}$ by $10 \mathrm{~mm}$ lead slit for the detector. Further collimation of the beam including down to $500 \mu \mathrm{m}$ by $500 \mu \mathrm{m}$ beam with a $500 \mu \mathrm{m}$ slit for the detector, produced approximately an $8 \% \mathrm{im}$ provement in full width half maximum (due to the reduced angular blurring). However, with the reduced scatter intensity, the resulting measurement times were of the order of 5 hours compared to 30 minutes, subjecting the X-ray system to overheating, and therefore the $1 \mathrm{~mm}$ by $10 \mathrm{~mm}$ geometry was used for the remainder of the experiment.

\subsubsection{Angular dispersive apparatus}

In addition to the energy dispersive measurements discussed previously, angular dispersive $\mathrm{X}$-ray diffraction using a $50 \mathrm{kV}$ molybdenum target X-ray tube and a 4-inch image intensifier (Hamamatsu Photonics Model C733610; refer to section 3.4.3) has been attempted with the apparatus shown schematically in Fig. 3.11. Collimation is provided by a cylindrical coppercoated lead collimator as per the energy dispersive apparatus. Initially a $1 \mathrm{~mm}$ diameter collimator was used, however the resultant X-ray intensity was found to be insufficient to generate a detectable diffraction image over the maximum acquisition time $(1000 \mathrm{~ms})$. This collimator was therefore later 


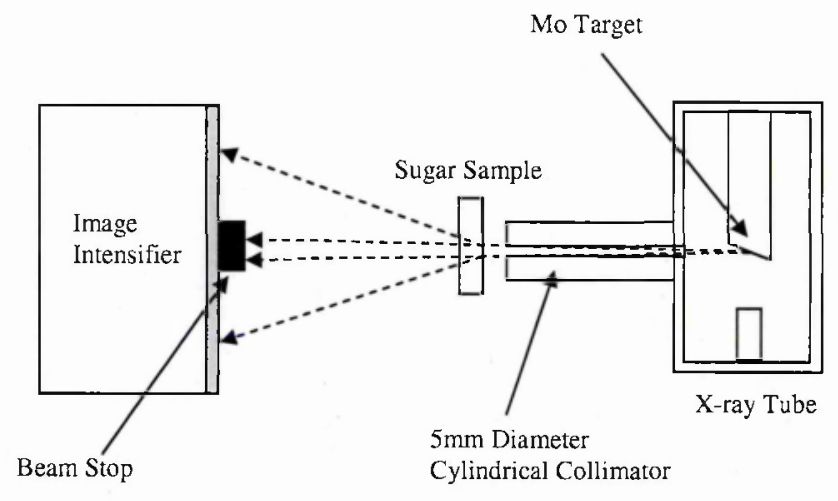

Figure 3.11: Schematic of the apparatus used for angular dispersive X-ray diffraction measurements.

replaced with a $5 \mathrm{~mm}$ diameter collimator, sacrificing resolution in order to generate a useful image. A $15 \mathrm{~mm}$ diameter lead beam stop was required in the centre of the image intensifier to cut out the primary beam from the centre of the intensity (diffraction) image, due to beam spread.

While ideally a monochromatic beam is required, as discussed in section 3.2, a quasi-monochromatic beam may be produced through application of a filter material. However at the time no suitable filter was available, the resulting intensity images are therefore subject to polychromatic blurring due to the multiple X-ray energies present in the beam.

While further angular dispersive work was initially planned including a refinement of that described in Appendix B [36], incorporating an electrical heating rig to replace the relatively crude butane/propane gas torch, and taking advantage of the improved Hamamatsu image intensifier. However, given the superior resolution of the energy dispersive system, and that the work was to be carried out under laboratory rather than industrial/field con- 
ditions, the focus of the remaining experiments was on further development of the energy dispersive technique.

\subsection{X-ray tubes}

\subsubsection{Production of X-rays}

$\mathrm{X}$-ray tubes provide the staple laboratory source for $\mathrm{X}$-rays given their relatively low cost and ease of setting up compared to a synchrotron source. $\mathrm{X}$-ray tubes produce relatively high intensities of $\mathrm{X}$-rays (when compared to isotope sources), both as a continuous bremsstrahlung spectrum and a superimposed spectrum of discrete characteristic X-rays as shown in Fig. 3.12. As briefly described in sections 3.2 .1 and 3.2 .2 , a number of different X-ray sets have been used for both energy dispersive (EDXRD) and angular dispersive (ADXRD) work. Initially $50 \mathrm{kV}$ molybdenum and silver target tubes were used for imaging low density samples in both energy dispersive and angular dispersive configurations. The beam current on these tubes is limited to $1 \mathrm{~mA}$ and $0.5 \mathrm{~mA}$ respectively, limiting their usefulness for later experiments on steel samples. An $80 \mathrm{kV}$ tungsten-target was also used for EDXRD work, generating an intense bremsstrahlung spectrum with the higher atomic number of tungsten (and high melting point of $3422^{\circ} \mathrm{C}$ allowing for a higher energy electron beam). The overall flux on this tube is however lower, with the beam current limited to $250 \mu \mathrm{A}$. The final and most significant $\mathrm{X}$-ray set used, was a $3 \mathrm{~kW}, 225 \mathrm{kV}$ tungsten-target tube, limited to $160 \mathrm{kV}$ and capable of exceptionally high flux due to a maximum beam current of $30 \mathrm{~mA}$ (at $100 \mathrm{kV}$, though limited initially to $10 \mathrm{~mA}$ due to overheating concerns). Dose- 


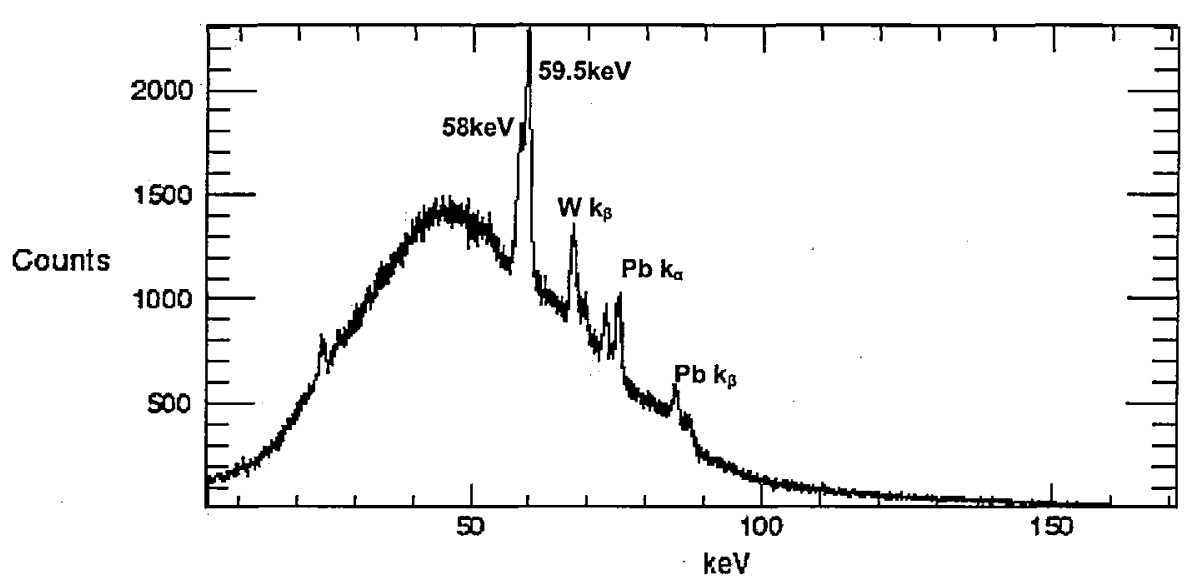

Figure 3.12: Spectrum obtained (using a high-purity germanium detector at a distance of $10 \mathrm{~m}$ with a pin-hole lead collimator) from HMS Sultan's tungstentarget X-ray tube operating at $160 \mathrm{kV}$. The bremsstrahlung continuum extends across the full range from $0 \mathrm{keV}$ to $160 \mathrm{keV}$. While a number of characteristic peaks are visible, the most prominent in the spectrum is the tungsten $K_{\alpha}$ doublet (58 to $59.5 \mathrm{keV}$ ) produced from the $L_{I}, L_{I I}$ subshells.

rate and shielding concerns are particularly prevalent with this set, given that the absorbed dose in the primary (collimated beam) reaches over $1 \mathrm{~Gy} / \mathrm{sec}$ $(100 \mathrm{kV} / 10 \mathrm{~mA}$ operation) at the collimator aperture. A near identical set was provided at HMS Sultan's laboratory, where due to improved local shielding, the set was cleared to run up to the full $225 \mathrm{kV}$.

The continuous spectrum is produced through interaction between electrons in the beam and nuclei in the target. An electron passing close to a nucleus undergoes a deflection producing a photon. The result of this sudden loss of energy is a rapid reduction in the electron's kinetic energy, in effect it has 'braked' (hence the term Bremsstrahlung radiation) due to the interac- 
tion. The energy transfer is continuous i.e. it may take any value up to and including the full electron kinetic energy, thus producing a broad spectrum of X-ray energies that tails off towards the maximum electron energy. In this case the energy is defined by the tube voltage $V$, where the maximum possible energy of a single accelerated electron in $\mathrm{keV}$ (and therefore the maximum X-ray energy) is equal to the tube voltage in $\mathrm{kV}$.

The total X-ray beam intensity is strongly dependent on tube voltage, and also the atomic number $Z$ and tube current $i$ such that for a thick target [37]:

$$
I \propto i Z V^{m}
$$

Where $m \approx 2$. The efficiency per electron is determined by:

$$
\eta=A i Z V
$$

Where $\mathrm{A}$ is a constant of $1.1 \times 10^{-9}$ per, volt. This demonstrates the poor efficiency of X-ray tubes; typically less than $2 \%$ of the total electron kinetic energy is transformed into X-rays, the remainder being converted into heat (hence the requirement for a high melting point material as the target).

The characteristic spectrum first discovered by C. G Barkla [38] (Nobel Prize 1917) is as a result of removal of an inner shell electron and subsequent de-excitation of higher electron shells. Because these shells $(K, L, M)$ correspond to discrete values of energy (principle quantum number $n=1,2,3$ ); discrete X-ray energies are produced. The energy of the resulting photon depends upon the shell or subshell that an electron de-excites from, for example 
the removal of a $K$-shell electron followed by de-excitation of an $L$-shell electron results in emission of a $K_{\alpha} \mathrm{X}$-ray. De-excitation of an $M$-shell electron to fill the vacancy in the $K$-shell results in emission of a $K_{\beta}$ X-ray. Because splitting of shells into subshells occurs due to the coupling of the intrinsic (spin) and orbital angular momenta of the electrons (i.e. the quantum number $j$, where $j=l \pm s$ ) for example, the $L$-shell splitting into $L_{I}, L_{I I}$, de-excitation from different subshells produces a further range of discrete $\mathrm{X}$-ray energies.

\subsubsection{Construction of X-ray tubes}

In terms of construction, the $50 \mathrm{kV}, 80 \mathrm{kV}$ and $225 \mathrm{kV} \mathrm{X}$-ray tubes used at both Surrey and HMS Sultan all share the same design principle, namely that of the stationary anode as illustrated in Fig. 3.13. The anode and cathode assembly are enclosed within an evacuated glass envelope, since the presence of gas molecules inside the tube presents both an electrical breakdown risk and a reduction of the maximum X-ray energy (due to electrons colliding with and ionising gas molecules). The glass construction of the envelope also prevents currents being induced due to the high voltage applied across the chamber.

The filament itself is usually tungsten as it has good thermionic properties and a low vapour pressure. Despite its high melting point $\left(3422^{\circ} \mathrm{C}\right)$ some evaporation of the filament does occur however, leading to the unavoidable build-up of a thin deposit of tungsten on the inner surface of the glass envelope and long-term risk of electrical leakage. It is desirable to produce a focused X-ray beam (a small 'focal spot') and therefore the filament is mounted inside a focusing cup, shaping the electric field between the cathode 


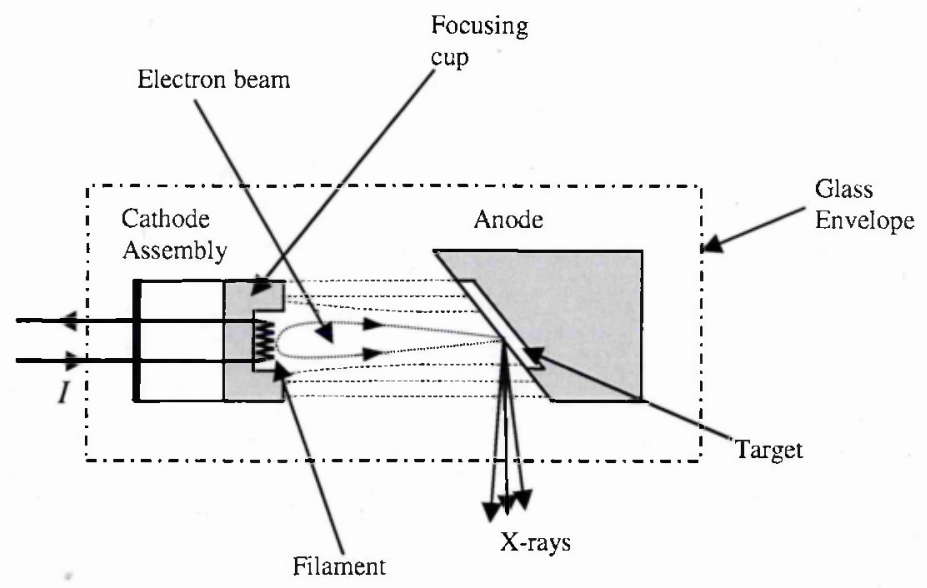

Figure 3.13: Simplified configuration of a stationary anode X-ray tube. It is important to distinguish the filament current $I$ (order of Amps) from the tube current resulting from thermionic emission (order of $\mathrm{mA}$ ). The focusing cup shapes the electric field so that electrons are focused into a thin rectangle on the target. X-rays are produced isotropically inside the anode, however only the $\mathrm{X}$-ray paths which pass through the tube exit window are shown.

and anode to ensure the electron beam is deposited into a small area of the target. The focusing cup is constructed from a material with relatively poor qualities as a thermionic emitter (e.g. stainless steel) to prevent broadening of the resultant focal spot by stray thermionic electrons. Dual focus X-ray tubes consist of two different sized filaments for lower power, fine focus and full power, broad focus applications.

The beam of electrons produced is accelerated by the large electric field gradient into the target, positioned on the front face of the anode. A variety of target materials are used. In low power tubes silver or molybdenum may form the target. In most higher power applications however, it is tungsten that is 
used given its high atomic number (desirable for producing high intensities of X-rays) and high melting point. The thermal conductivity of tungsten is another desirable property, allowing for rapid transfer of heat from the small target area to the copper anode block. For the smaller $50 \mathrm{kV}$ and $80 \mathrm{kV}$ tubes air cooling is sufficient for stable operation. The higher flux $225 \mathrm{kV}$ tubes however require dedicated water-cooling, including a chiller unit able to maintain the coolant temperature at $15^{\circ} \mathrm{C}$.

The actual focus of the electrons on the target is a thin rectangle (although it is referred to as a 'line focus'), however because the anode is inclined at an angle with respect to the X-ray beam, the effective focal spot is square. This configuration therefore has a number of advantages not least the distribution of heat over a wider area of the target (resulting in a lower temperature rise of the target/anode) than the effective focus would suggest. In addition, because the effective focus is smaller than the real focus, the filament may be relatively long allowing for a larger surface area and higher efficiency for thermionic emission.

Stationary anode X-ray tubes have power ratings of up to several $\mathrm{kW}$ where the tube current is of the order of several tens of $\mathrm{mA}$. The limiting factor to increasing this further is the ability to cool the copper anode block (melting point $1083^{\circ} \mathrm{C}$ ). The solution is the rotating anode, allowing for greater efficiency of cooling (as the beam is continuously presented with a freshly cooled surface) and therefore the use of higher tube currents. Rotating anode X-ray tubes are able to produce significantly higher intensities of $\mathrm{X}$-rays given the ability to operate at several tens of $\mathrm{kW}$ with tube currents of several hundred $\mathrm{mA}$. 


\subsection{Detectors}

The development of detectors for applications rainging from Particle Physics to X-ray/medical imaging and Nuclear Physics is an ongoing and highly active area of research in which spin-offs from one area, (e.g. medipix from CERN [39]) are particularly relevant for another (e.g. X-ray imaging).

In terms of detector systems used for X-ray scattering work and angular dispersive measurements, these are generally based upon systems already developed and in use for medical imaging and radiography, where the need is for high quality digital intensity images to replace photographic film. Energy dispersive work utilises readily available energy-resolving semiconductor based devices, for example high-purity germanium as used in spectroscopic studies, and developments in silicon detector technology driven partially by developments and spin-offs from large-budget particle physics research such as the extensive detector stations at CERN. The expansion in the use of silicon detectors over other semiconductor detectors can to a large extent be attributed to the rapid growth of the global electronics industry, worth \$257 Billion in 2004 (European Electronic Component Manufactures Association [40]), and the adoption of process techniques originally developed for electronics-grade silicon.

\subsubsection{Introduction to germanium and other semicon- ductors}

As with silicon the earlier germanium detectors were lithium-drifted devices, where permanent cooling is required to maintain the device at $77 \mathrm{~K}$ in order to 
prevent the lithium migrating out of the depletion region. The production of $\mathrm{Ge}(\mathrm{Li})$ detectors has now largely ceased, with manufacturers concentrating on High-Purity Germanium (HPGe) detectors which only require cooling during operation.

The size of the depletion region formed is dependant on the level of impurities in the germanium and the reverse bias applied. For detectors, complete depletion of the semiconductor wafer used improves the charge collection efficiency and the sensitive volume of the detector. For a bias of $1000 \mathrm{~V}$ and an impurity concentration of 1 part in $10^{12}$ the depletion region is approximately $10 \mathrm{~mm}$ thick [41]. It is therefore desirable to use as high a purity (i.e. intrinsic) germanium as possible, achieved through repeatedly melting localised areas of the germanium to remove the more soluble impurities from the material. The resulting semiconductor is either mildly $p$-type $(\pi)$ or mildly $n$-type $(\nu)$, depending on the remaining impurities.

In a typical planar configuration a disc of high purity germanium is diffused with an $n^{+}$contact on one face usually through the evaporation of lithium ions onto the surface. On the opposing face a $p^{+}$contact is formed, typically through doping with boron atoms. A sufficiently large reverse bias is then applied such that a positive potential is connected to the $n^{+}$contact, depleting the detector fully and providing a uniform electric field to minimise the charge collection time.

The maximum active volume of a planar configuration detector is limited by the thickness of the depletion region (that is of the order of $1 \mathrm{~cm}$ ). By using an axial configuration as illustrated in Fig. 3.14 the detector volume may be increased for a given depletion depth. 


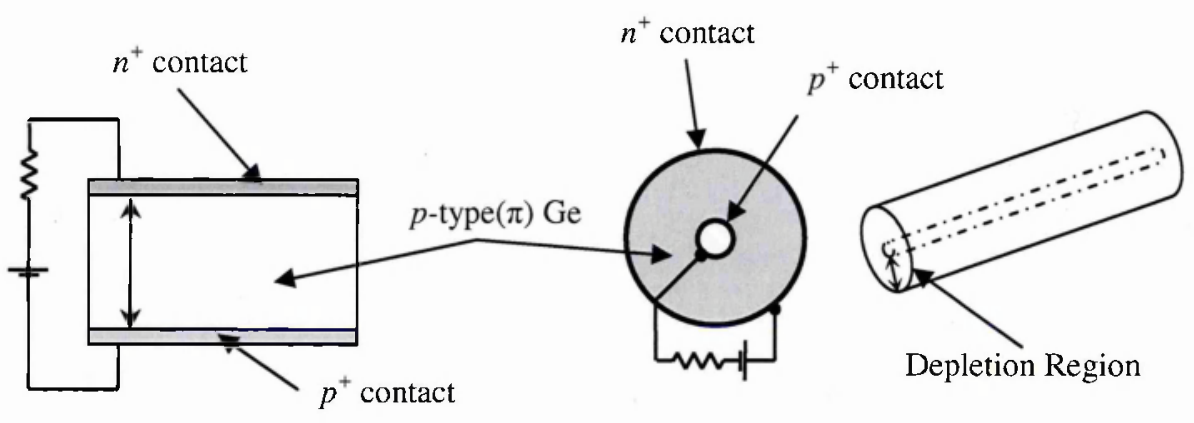

(a)

(b)

Figure 3.14: Configuration of (a) planar and (b) coaxial HPGe detectors.

HPGe detectors do not require permanent cooling as for lithium drifted germanium (where temperature rises above $77 \mathrm{~K}$ begin to destroy the detector effectiveness). However given the small bandgap in germanium $(0.7 \mathrm{eV})$, liquid nitrogen-cooling is still required during operation to minimise noise that is caused by thermally excited electrons reaching the conduction band.

The key characteristic of germanium detectors is their exceptional energy resolution (a consequence of the small bandgap), for example a typical, small planar detector has an energy resolution of the order $150 \mathrm{eV}$ at $5.9 \mathrm{keV}$. For large coaxial detectors at measured gamma-ray energies of $1.3 \mathrm{MeV}$, energy resolution as good as $1.7 \mathrm{keV}$ is achievable [41].

High-purity germanium detectors are considered the 'gold standard' in detectors with respect to their energy resolution and applicability to spectroscopic studies. The chief disadvantage however is the requirement for bulky liquid nitrogen cooling. Silicon detectors such as the $\mathrm{Si}(\mathrm{Li})$ have the advantage of being (in principle) operable at room temperature, however 
this is at the expense of ultimate energy resolution. Given that silicon also has a lower atomic number than germanium ( $Z=14$, compared to $Z=32$ ), it also has lower efficiency for photoelectric absorption, and thus a lower efficiency for detecting higher energy X-rays and gamma-rays. The search for room-temperature semiconductor detectors capable of spectroscopic studies with X-rays and gamma rays has led to the development of compound detectors, the most successful (i.e. commercially available) being based largely on Cadmium Telluride (CdTe) and derivatives such as Cadmium Zinc Telluride (CZT).

These designs exploit the higher atomic number of cadmium $(Z=48)$ and tellurium $(Z=52)$ to improve the efficiency for photoelectric absorption, thus allowing for smaller detector volumes. The band gap of these materials is relatively large compared to silicon or germanium (of the order of $1.5 \mathrm{eV}$ ) thereby reducing noise due to thermally excited electrons. The relaxed constraints on operating temperature (around 230K) for low energy X-rays allows for the use of Peltier cooling (with regards to higher energy gamma rays room temperature operation is possible), resulting in a more compact, robust device.

The drift velocity for holes with compound semiconductors such as $\mathrm{CdTe}$ is however less than one tenth of that of electrons [42][43]. As a result, the energy resolution obtainable with these devices is not comparable with that obtained with silicon or germanium. Considering $122 \mathrm{keV}$ gamma rays, older $\mathrm{CdTe}$ detectors may achieve a resolution of $3.2 \mathrm{keV}$ compared to $400 \mathrm{eV}$ [41] for germanium. Compound semiconductors also suffer greatly from defects in the material from the manufacturing process compared to for example 
high-purity germanium.

CZT devices initially yielded improved resolution over CdTe and do not suffer the effects of electrons being trapped in deep acceptor states, a process which leads to a decrease in charge collection efficiency and counting rate in CdTe. However in more recent years developments of CdTe, particularly in terms of the contacts, have yielded improvements in sensitivity and resolution (through reduced leakage current) such that for $1-2 \mathrm{~mm}$ thick detectors (Amptek XR-100T range for example), these now out-perform CZT devices with resolutions of $290 \mathrm{eV}$ at $5.9 \mathrm{keV}$ and $850 \mathrm{eV}$ at $122 \mathrm{keV}$ [44].

\subsubsection{Devices used for energy dispersive XRD}

For energy dispersive X-ray diffraction work three separate devices have been used. Initially the sole detector available was an Amptek XR-100T CZT (now only available as a CdTe detector) as shown in Fig. 3.15. This device consists of $2 \mathrm{~mm}$ thick CZT with a thin beryllium window, built in pre-amp and Peltier cooling to reduce thermally induced noise. The quoted total intrinsic detection efficiency [45] in the range of energies encountered (approximately $30 \mathrm{keV}$ up to $100 \mathrm{keV}$ ) is $99.16 \%$ down to $86.46 \%$. Probability of photoelectric interaction is quoted as $99.16 \%$ down to $82.68 \%$. In Fig. 3.16 this is compared directly to the established XCOM data for photon interactions in matter. For this detector the zinc fraction of the CZT is typically 0.1 i.e. $\mathrm{Cd}_{0.9} \mathrm{Zn}_{0.1} \mathrm{Te}$. Using this element ratio, and for an energy range of $30 \mathrm{keV}$ to $100 \mathrm{keV}, \mathrm{XCOM}$ yields a photoelectric cross section from $1.94 \mathrm{~cm}^{2} / \mathrm{g}$ to $1.36 \mathrm{~cm}^{2} / \mathrm{g}$ and a total attenuation cross section of $1.96 \mathrm{~cm}^{2} / \mathrm{g}$ to $1.56 \mathrm{~cm}^{2} / \mathrm{g}$ i.e. a photopeak efficiency of $99.0 \%$ at $30 \mathrm{keV}$, down to $87.2 \%$ at $100 \mathrm{keV}$. 


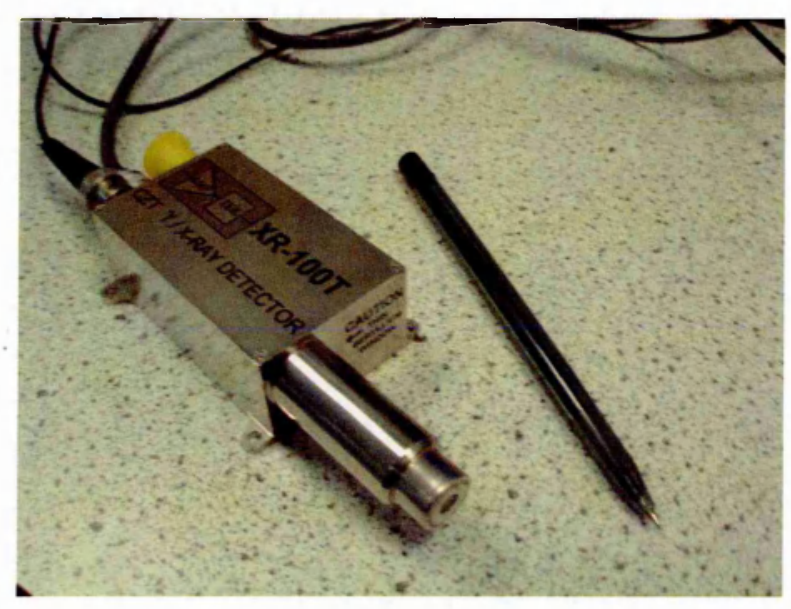

Figure 3.15: The Amptek XR-100 family of detectors are exceptionally compact as visible in the above photograph. There is no requirement for liquid nitrogen cooling, and the complete device is more robust and portable than for example a HPGe detector with it's bulky cryostat.

The quoted manufacturer's energy resolution for this detector is not readily available, as the $2 \mathrm{~mm} \mathrm{CZT}$ has been replaced with $1 \mathrm{~mm}$ CdTe with superior sensitivity (due to superior charge transport properties of CdTe and the use of higher bias voltage) and resolution. However experimental work indicates the XR-100 CZT devices are capable of a resolution of $780 \mathrm{eV}$ at $59 \mathrm{keV}\left({ }^{241} \mathrm{Am}\right)$ compared to the CdTe resolution of $600 \mathrm{eV}$ at $59 \mathrm{keV}$ [41].

The second device available for EDXRD work was an Ortec GLP-10180/07P planar HPGe detector. This consists of $10 \mathrm{~mm}$ thick $p$-type germanium with a $0.7 \mathrm{~mm}$ thick lithium diffused $n^{+}$contact and a $125 \mu \mathrm{m}$ beryllium window. The resolution at $5.9 \mathrm{keV}$ is quoted as $180 \mathrm{cV}$ and $485 \mathrm{eV}$ at $122 \mathrm{keV} \mathrm{[46].} \mathrm{Refer-}$ ring again to XCOM, the photoelectric cross-section ranges from $13.4 \mathrm{~cm}^{2} / \mathrm{g}$ at $30 \mathrm{keV}$ to $0.384 \mathrm{~cm}^{2} / \mathrm{g}$ at $100 \mathrm{keV}$, while the total interaction cross section 

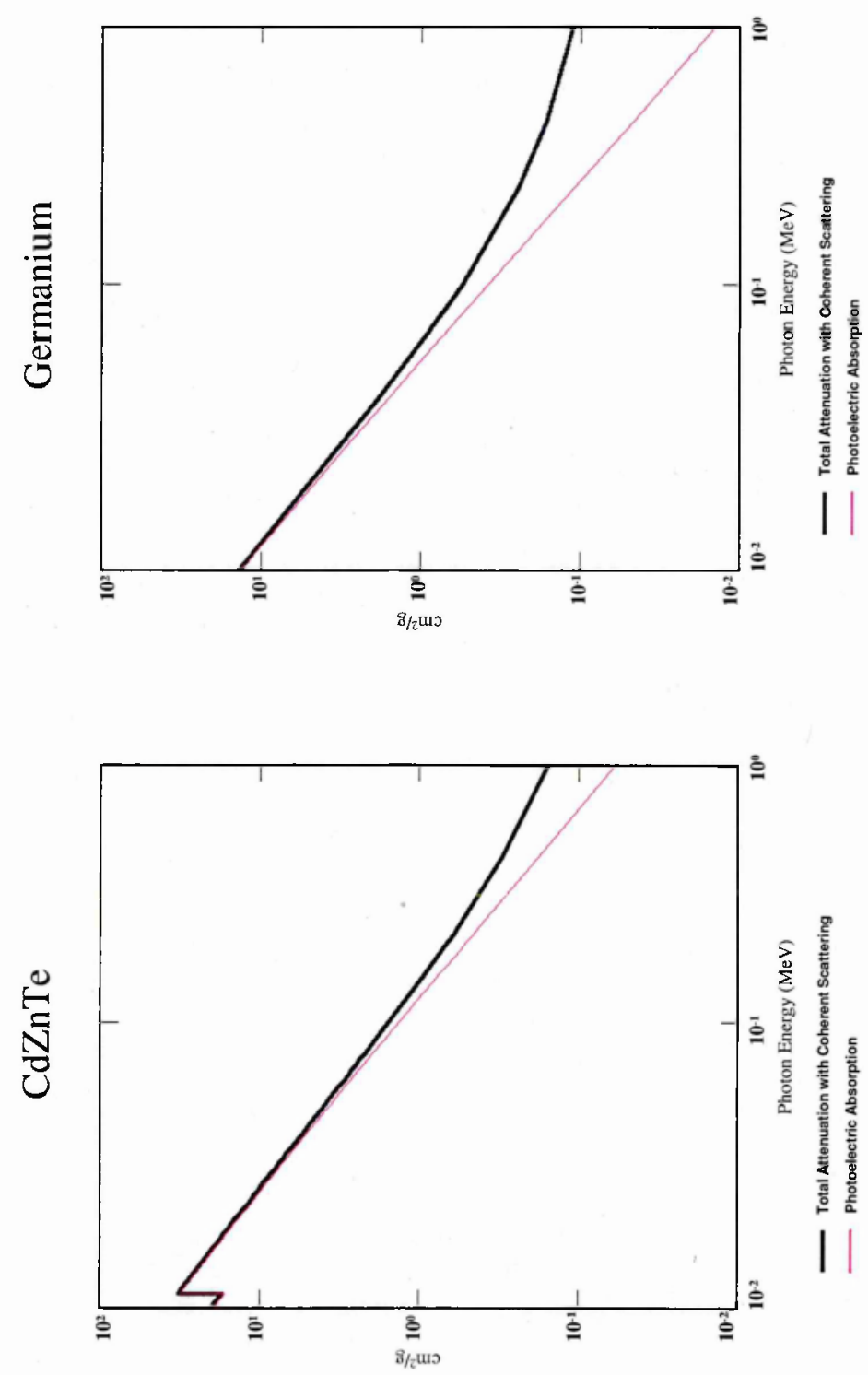

Figure 3.16: Using the established XCOM data, the photoelectric absorption cross section is compared with the total attenuation cross section, giving the photopeak efficiency at energies ranging from 10 to $1000 \mathrm{keV}$. As shown, the efficiency for CZT remains close to 100\% across the energy range used in the EDXRD experiments. Germanium, with it's lower atomic number compared to the compound semiconductors such as CZT, has a significantly lower photopeak efficiency towards the $100 \mathrm{keV}$ limit of X-ray energies used in these experiments. 
ranges from $13.8 \mathrm{~cm}^{2} / \mathrm{g}$ to $0.555 \mathrm{~cm}^{2} / \mathrm{g}$, yielding a photopeak efficiency of $96.8 \%$ at $30 \mathrm{keV}$ down to $69.2 \%$ at $100 \mathrm{keV}$.

Following a failure of the pre-amp, and possible loss of vacuum, this detector was replaced with a DSG Systems GLP-06 165/05-P planar HPGe detector with near identical resolution and sensitivity.

\subsubsection{Devices used for angular dispersive XRD}

Angular dispersive XRD has only been briefly investigated for use in this project. The Hamamatsu photonics C7336-10 device consists of a 4-inch image intensifier with caesium iodide screen and $0.5 \mathrm{~mm}$ aluminium input window, coupled to a CCD camera (as shown in Fig. 3.17) with an effective area of $70 \times 52$ millimetres consisting of 650 by 580 pixels.

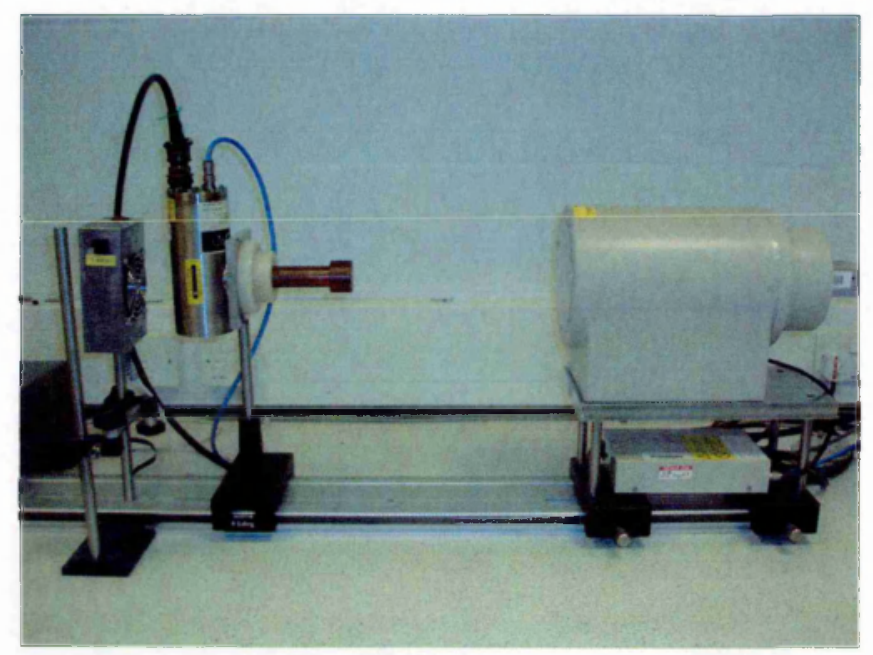

Figure 3.17: Shown here is the image intensifier (right) used for preliminary ADXRD work. Also shown to the left is the $50 \mathrm{kV}$ X-ray tube complete with copper-coated lead collimator. 


\section{Chapter 4}

\section{Experimental results and discussion}

\subsection{Introduction}

As discussed in chapter 1 the primary aim of the current work is to explore the use of transmission geometry X-ray diffraction for materials purposes. In terms of the Naval Nuclear Propulsion Program (NNPP), of particular interest is the effect of stress and corrosion (Stress Corrosion Cracking) upon the structure of austenitic stainless steel and the ability to accurately image such changes (for example, lattice spacing changes). Transmission geometry diffraction requires the generation of X-rays of sufficient energy to overcome the attenuation effects of any sample under investigation. It has been previously demonstrated [35] (refer to Appendix A) that an existing $225 \mathrm{kV}$ tungsten target X-ray tube at HMS Sultan's Nuclear Department is suitable for performing transmission diffraction measurements. In addition to 
this apparatus, a $225 \mathrm{kV}$ tungsten tube (Comet MXR225/22) is available at the University of Surrey Physics department for the purpose of both energy dispersive and angular dispersive measurements (see Appendix B) in steel samples.

Section 4.2 describes preliminary work carried out with an angular dispersive apparatus using an X-ray image intensifier with a $50 \mathrm{kV}$ X-ray tube. This did not progress beyond imaging low- $Z$ samples due to the time constraints and the focus shifting over to the energy dispersive configuration once the $225 \mathrm{kV}$ tube was fully operational.

In section 4.3 energy dispersive measurements taken on austenite and ferrite-phase samples are discussed. Development initially focused on the system resolution and optimising the geometry based upon 3mm thick, lowalloy ferritic and austenitic stainless steel samples using the $225 \mathrm{kV}$ X-ray tube. This is also useful for comparison to the ADXRD work carried out on similar samples in Appendix B [36]. Following this phase of work, austenitic stainless steel samples subjected to extreme plastic deformation were investigated. To complement this work a dynamic system was later developed, whereby samples could be investigated with EDXRD whilst being subjected to elastic strain up towards the yield point of 7 kilonewtons. The final investigations carried out involved a preliminary map of an austenite sample, cut from a pipework specimen, and loaded to generate a residual stress field. This sample was then quenched at $77 \mathrm{~K}$ in an attempt to form the martensite phase (refer to Fig. 4.18 with regards to description of the martensite phase) in the steel.

Finally in section 4.4 an experiment conducted at the European Syn- 
chrotron Radiation Facility for the purposes of investigating residual stress and crack growth in the same ferritic steel is described. This investigation, while focused on verifying a model of fatigue-crack propagation, proves a useful comparison between energy dispersive measurements on synchrotronbased and table-top systems.

\subsection{Angular dispersive X-ray diffraction re- sults}

Parallel to the use of the energy dispersive configuration for transmission $\mathrm{XRD}$, use of a quasi-monochromatic source and position sensitive (area) detector had been briefly investigated and is included here for completeness.

The results for sugar are shown in Fig. 4.1. Given the weak nature of the resulting intensity images, the image intensifier capture time was set to the maximum of $1000 \mathrm{~ms}$ with the gain increased to 19.1 . In addition to the diffraction image, an image was captured while no X-rays were being generated to perform a background subtraction and therefore improve the contrast. Despite the lack of beam filtration and the less than ideal parameters used, a diffraction pattern is clearly visible. In sugar, although the crystals are orientated in a random fashion, there are not a sufficient number to produce continuous rings (as seen in section 1.2 with steel) and as a result, a large number of individual diffraction spots are produced.

Attempts have also been made to produce images for $\mathrm{PVG} / \mathrm{SiO}_{2}$ and aluminium; the latter is of particular interest as a relatively simple series of diffraction rings are produced, allowing for radial integration of the intensity 


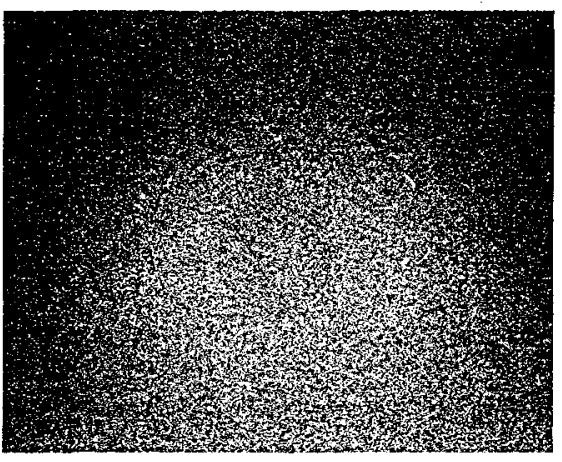

(a)

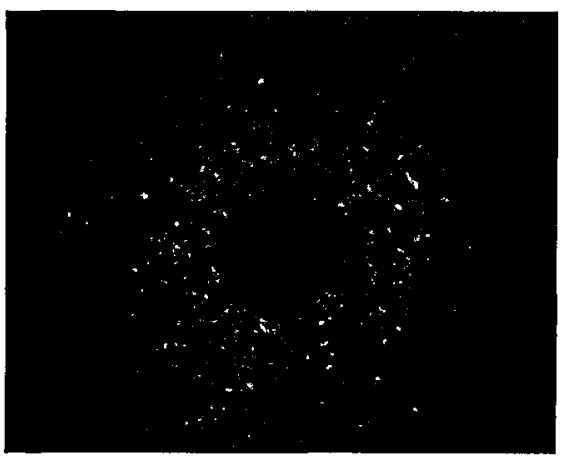

(b)

Figure 4.1: (a) Diffraction image produced from the scattering of X-rays from sugar. (b) The contrast of the image was improved through subtraction of the background, leading to clearly defined diffraction spots. The outline of the beam stop is visible in the centre of the image.

image to produce a plot of intensity vs. scattering angle. However given the relatively low intensity of X-rays used, no definable diffraction patterns could be derived for either material.

Given time constraints, once the new $225 \mathrm{kV}$ X-ray tube was installed the focus switched back to the EDXRD technique and no further measurements were carried out using the image intensifier. However, a separate investigation referred to in Appendix B, demonstrates the capability of an ADXRD system using a $225 \mathrm{kV} \mathrm{X}$-ray tube for the imaging of ferrite and austenitephase steel, in particular the changes produced as ferritic steel is heated towards the phase transformation temperature (approximately $800^{\circ} \mathrm{C}$ ) and annealed to the austenite phase. Whilst the system resolution will not match that of the energy dispersive configuration using HPGe detectors and CZT, it does allow for a robust apparatus, that would be more suited to extreme 
environmental conditions.

\subsection{Energy dispersive X-ray diffraction results}

Prior to obtaining a $225 \mathrm{kV}$ X-ray tube at Surrey, transmission diffraction measurements on steel samples were not possible given the limited flux and energy provided by the $50 \mathrm{kV}$ and $80 \mathrm{kV}$ X-ray tubes. Experiments carried out from late 2004 until December 2005 were limited to low-Z samples that, while not directly applicable to transmission diffraction in steel, proved useful in setting up the geometry of the system and testing the system resolution.

\subsubsection{Energy dispersive XRD on low density materials}

Initial measurements focused on the scattering from thin samples of sugar or a polyviologen-silica hybrid ( $\mathrm{PVG} / \mathrm{SiO}_{2}$ ) nanopowder, where the geometry is such that differential attenuation effects are minimised. All measurements taken have been at a scattering angle of $5^{\circ}$, with constant X-ray tube running conditions and an acquisition time of 1800 seconds for each measurement. A selection of the spectra obtained are shown in Fig. 4.2. Calibration was carried out using a standard variable $\mathrm{X}$-ray source $\left({ }^{241} \mathrm{Am}\right.$ source with various targets for the production of characteristic X-rays from $8 \mathrm{keV}$ copper $K_{\alpha}$ $\mathrm{X}$-rays up to $44 \mathrm{keV}$ terbium $K_{\alpha} \mathrm{X}$-rays). Due to the complexity of sugar (i.e. multiple lattice spacings) the resulting spectra consist of multiple characteristic peaks. Referring to plots (a), (b) and (c) in Fig. 4.2 it may at first appear as though the characteristic peaks are shifting energy. However, closer inspection reveals that they are infact constant throughout, but it is 


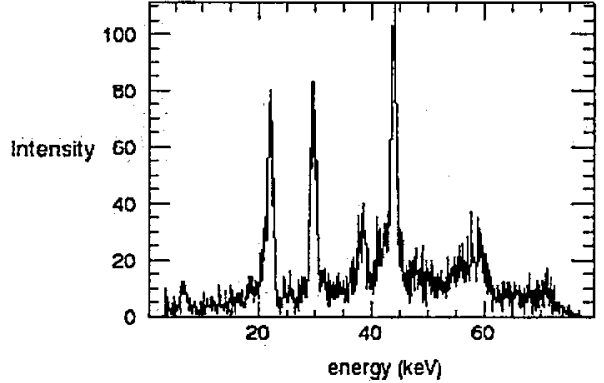

(a)

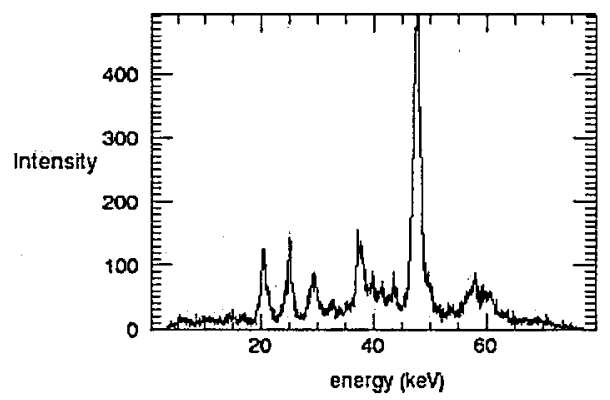

(c)

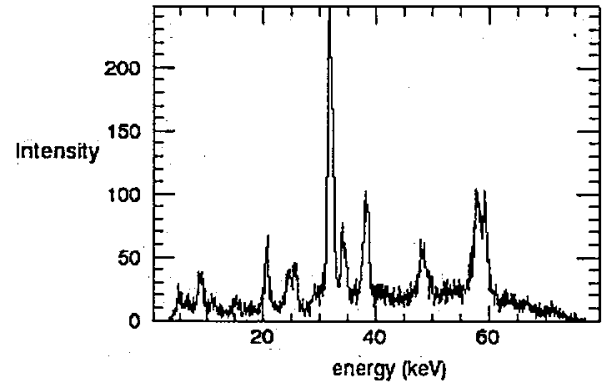

(b)

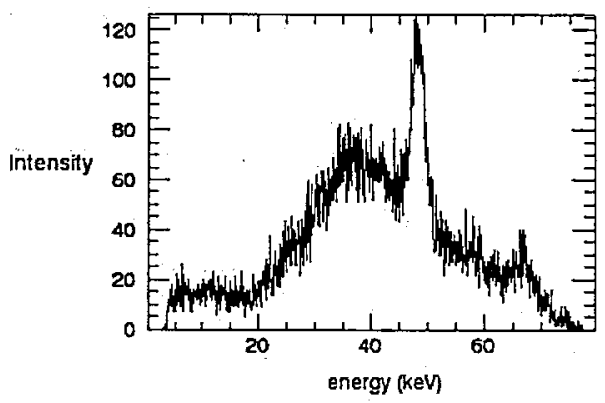

(d)

Figure 4.2: The most commonly imaged substance thus far is sugar as shown in (a), (b) and (c). The intensity of the characteristic peaks varies significantly between measurements. Shown in (d) is the spectrum for $\mathrm{PVG} / \mathrm{SiO}_{2}$.

the relative intensities of the peaks that change significantly. This is due to the nature of the sample; a simple loose-packed volume of granulated sugar, where the intensity changes are the manifestation of sugar crystals changing orientation within the sample due to small movements (vibration for example). $\mathrm{PVG} / \mathrm{SiO}_{2}$ has a single definable peak, unfortunately the sample used is known to have a small iron contamination and, combined with the resolution achievable, measurements of the lattice spacings in the sample were not obtainable. 


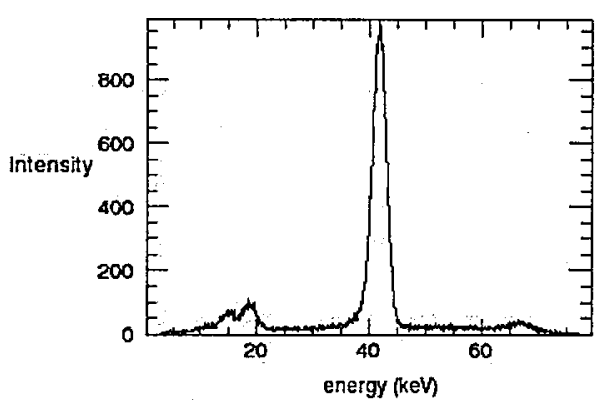

(a)

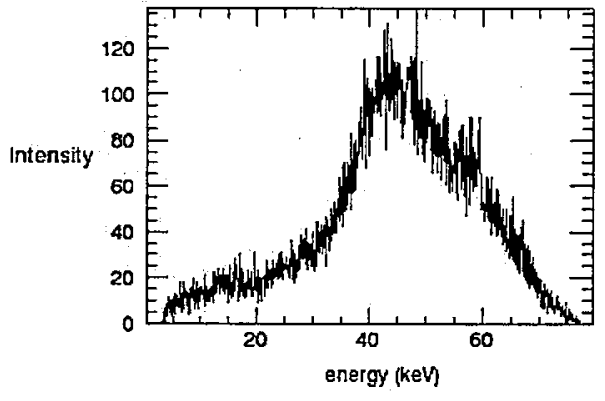

(b)

Figure 4.3: Other substances investigated include (a) graphite, with a single characteristic peak corresponding to the $3.35 \AA\left(d_{002}\right)$ lattice spacing and, (b) water with a broad featureless spectrum.

Also included in Fig. 4.3 are the spectra for graphite and water. As expected the spectrum generated from water is broad and featureless as a result of interference of scattered X-rays from neighbouring water molecules. With graphite a single prominent peak is visible at approximately $40 \mathrm{keV}$. Using a calibration in terms of the momentum transfer parameter $x$; this peak corresponds to $x=0.15 \AA^{-1}$ and as discussed in section 3.2 the associated lattice spacing is obtained from $x=\frac{1}{2 d}$ yielding the result $3.33 \pm 0.04 \AA$. When considering the limited resolution of the system this compares well with the accepted powder diffraction value of $3.35 \AA$ for the $d_{002}$ plane [29].

Further to the initial measurements on sugar, a custom built sampleholder, visible in Fig. 4.4 and capable of withstanding temperatures up to approximately $300^{\circ} \mathrm{C}$, has been used in an attempt to detect the increase in lattice spacings with increased temperature. The sample holder itself was mounted in the centre of the diffraction apparatus on top of an electrical heat- 


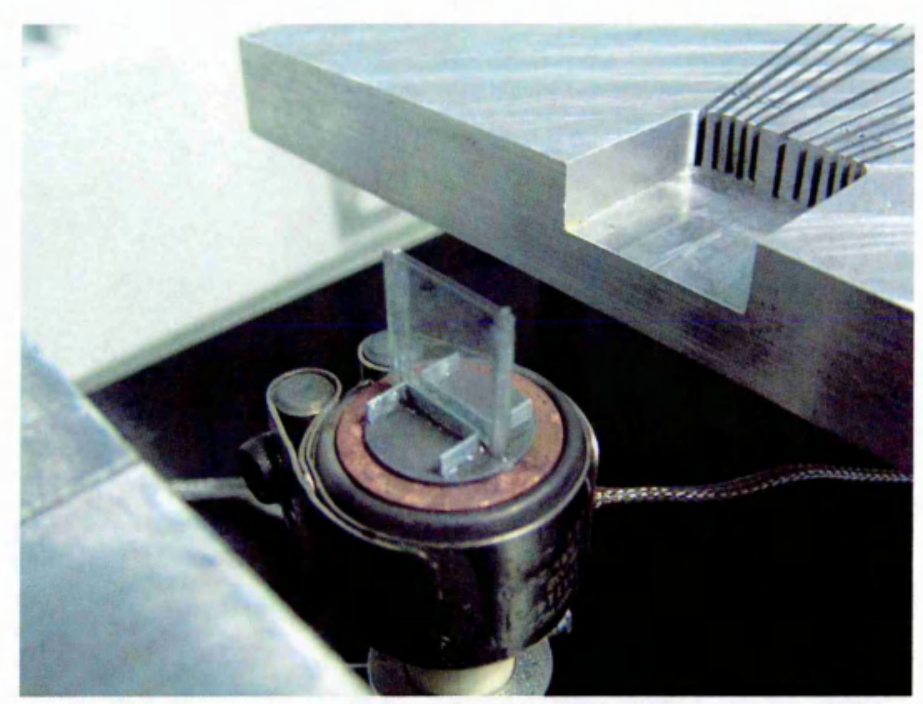

Figure 4.4: Photograph of the sample holder used for heating sugar up to $160^{\circ} \mathrm{C}$ mounted on top of the electrically heated stage.

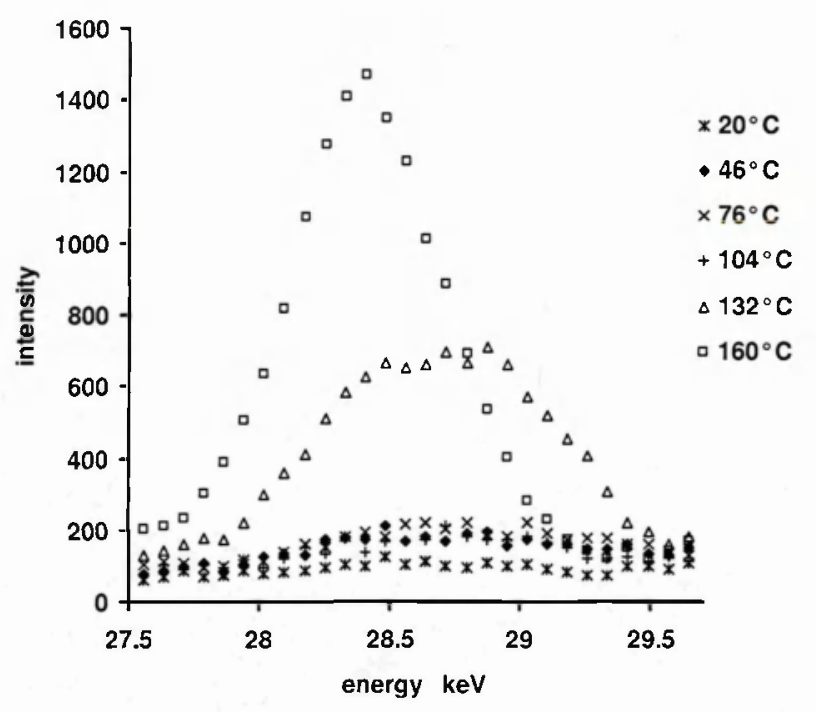

Figure 4.5: Intensity vs. energy plot for the region $27 \mathrm{keV}$ to $30 \mathrm{keV}$ for sugar at temperatures of $20^{\circ} \mathrm{C}$ through to $160^{\circ} \mathrm{C}$. 
ing stage coupled to a Eurotherm 2416 controller/set-point programmer. The most prominent changes were visible in the region $27 \mathrm{keV}$ to $30 \mathrm{keV}$, shown in Fig. 4.5 for temperatures increasing from $20^{\circ} \mathrm{C}$ through to $160^{\circ} \mathrm{C}$. The changes are subtle at lower temperatures, with more significant differences occurring between the temperatures of $132^{\circ} \mathrm{C}$ and $160^{\circ} \mathrm{C}$ (approaching the melting point of $186^{\circ} \mathrm{C}$ ). It should be noted however that with the demonstrated resolution, the system is not capable of accurately measuring the associated lattice spacings.

\subsubsection{Energy dispersive XRD on steel}

The low-alloy ferritic steel, and austenitic stainless steel (304L) used in these investigations have been cut into a variety of sample shapes as shown in Fig. 4.6. The long 'billet' austenite-phase samples are a standard, 3mm thick design used with the Monsanto tensometer (see section 3.2.1). The circular and oval shaped stainless samples are cut from specimens of pipework and have been polished on one side. These samples have then been loaded compressively, deforming them in various degrees from a purely circular shape, to oval shape, producing the desired residual stress field. The ferrite-phase samples are cut from a larger block of steel and slowly machined down (minimising induced stresses) to varying thicknesses of $1 \mathrm{~mm}$ through to $6 \mathrm{~mm}$.

Initial measurements focused on $1 \mathrm{~mm}$ and $3 \mathrm{~mm}$ thick samples of austenite and ferrite-phase steel and the collection of energy-space spectra over the full range of scattering angles available with the apparatus. The spectra collected from angles $3^{\circ}$ to $12^{\circ}$ over a period of half an hour (1800 seconds precisely) for $3 \mathrm{~mm}$ thick samples are shown in Fig. 4.7 to Fig. 4.10 , with the X-ray 


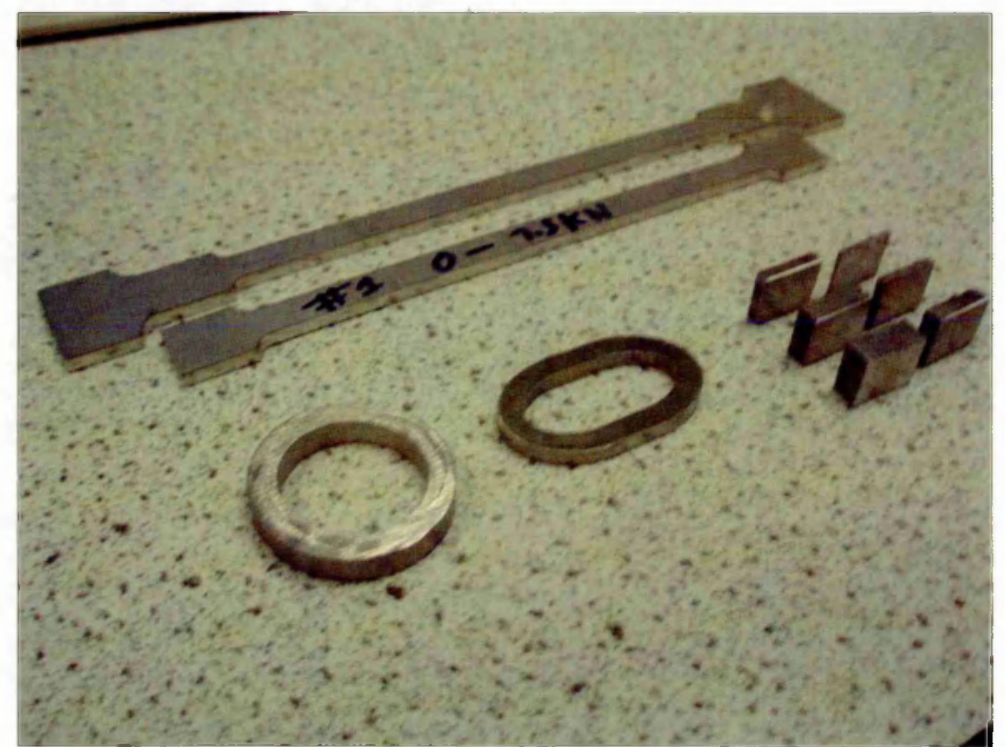

Figure 4.6: A selection of the steel samples used for EDXRD. The six roughly square samples on the right are low-alloy ferritic steel of thickness $1 \mathrm{~mm}$ through $6 \mathrm{~mm}$. The long samples are the $304 \mathrm{~L}$ austenitic stainless steel 'billets', designed specifically for use with the Monsanto tensometer, where a collar extends over either end locking the sample in place. The final samples are those cut from stainless steel pipework (again 304L), and subjected to a compressive load to introduce a residual stress.

tube operated at $100 \mathrm{kV}$ and $10 \mathrm{~mA}$ throughout. Measurements of $1 \mathrm{~mm}$ and $3 \mathrm{~mm}$ steel reveal that while differential attenuation of the scattered beam occurs, it is still possible to adequately resolve the diffraction peaks in order to obtain lattice spacings. Over the full range of angles a scattering angle of $9^{\circ}$ appears to be optimum, particularly for resolving the structure of ferritephase stcel. For example, with a $3 \mathrm{~mm}$ steel sample and at a scattering angle of $9^{\circ}$, all calculated lattice spacings agree with the X-ray powder diffraction 


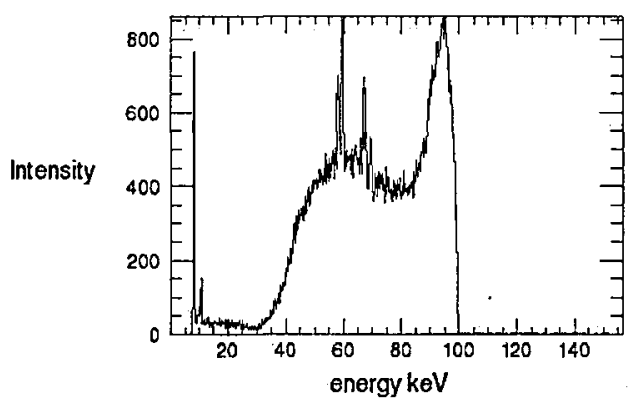

(a)

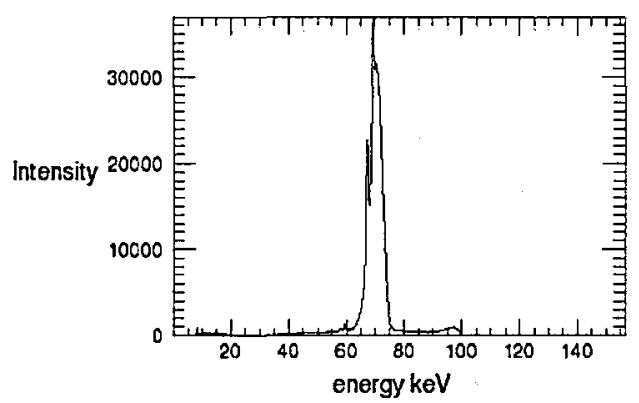

(c)

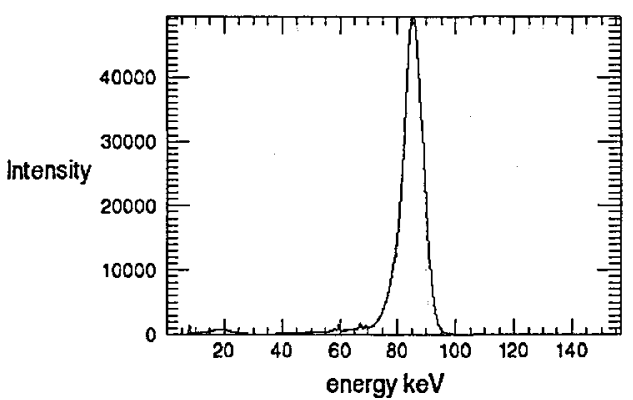

(b)

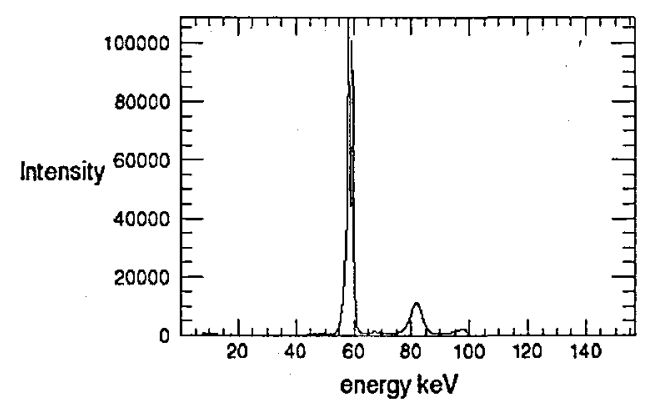

(d)

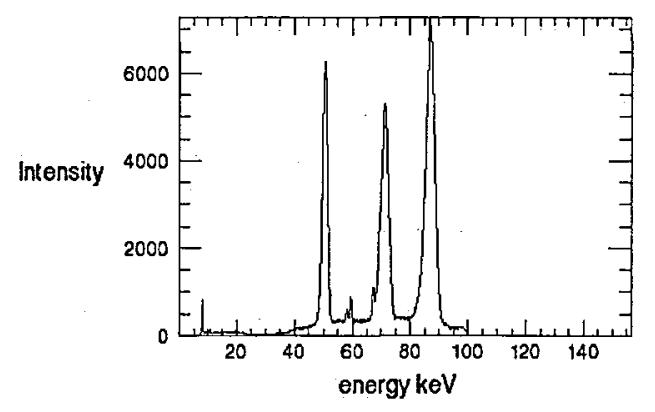

(e)

Figure 4.7: Spectra obtained for $3 \mathrm{~mm}$ ferrite-phase steel at scattering angles of; (a) $3^{\circ}$, (b) $4^{\circ}$, (c) $5^{\circ}$, (d) $6^{\circ}$ and (e) $7^{\circ}$. 


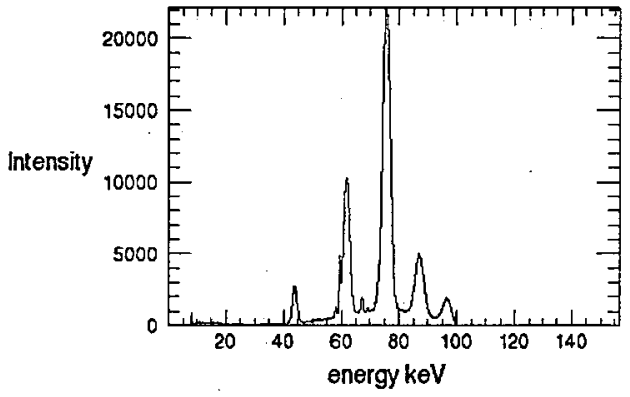

(a)

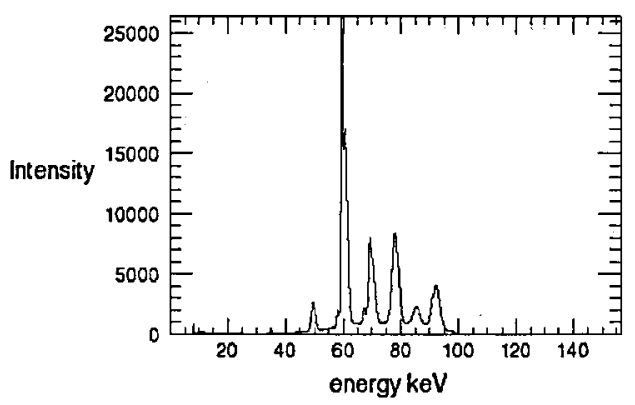

(c)

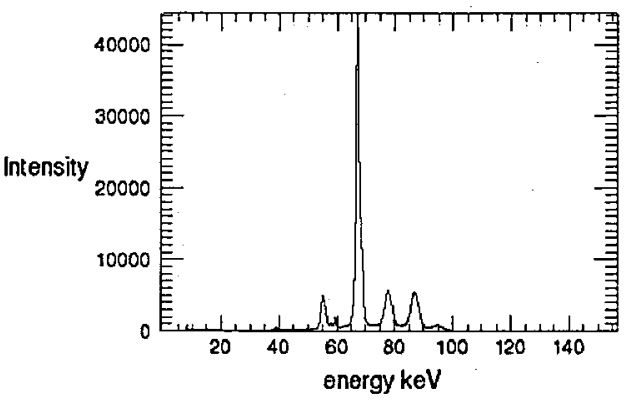

(b)

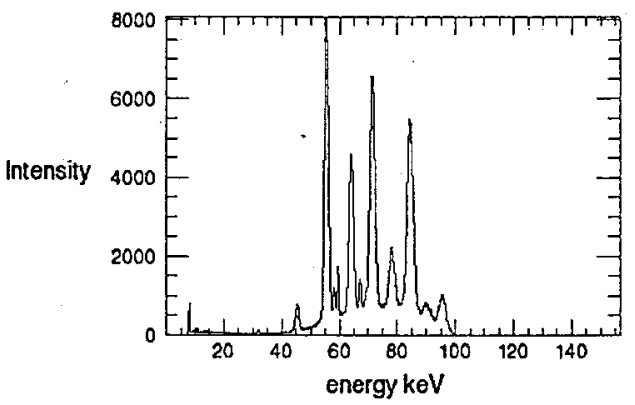

(d)

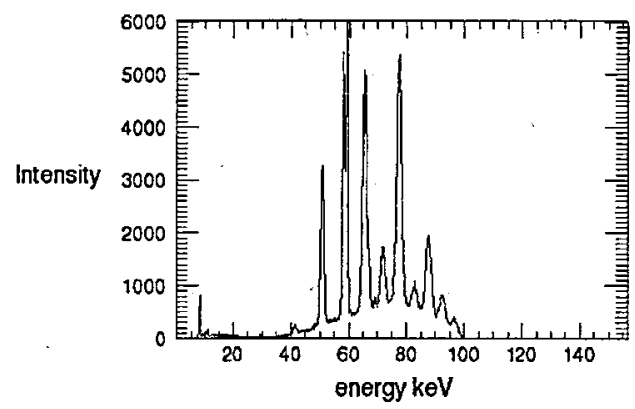

(e)

Figure 4.8: Spectra obtained for $3 \mathrm{~mm}$ ferrite-phase steel at scattering angles of; (a) $8^{\circ}$, (b) $9^{\circ}$, (c) $10^{\circ}$, (d) $11^{\circ}$ and (e) $12^{\circ}$. 


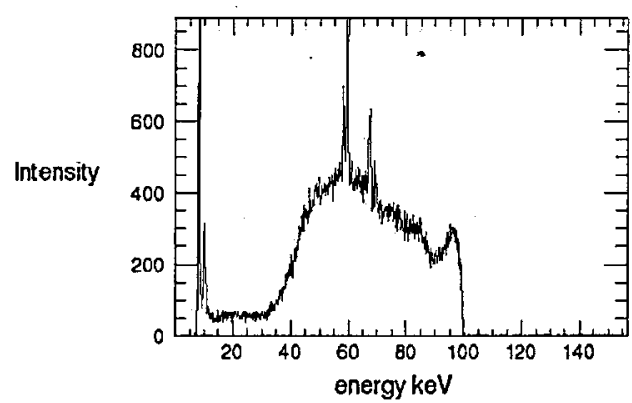

(a)

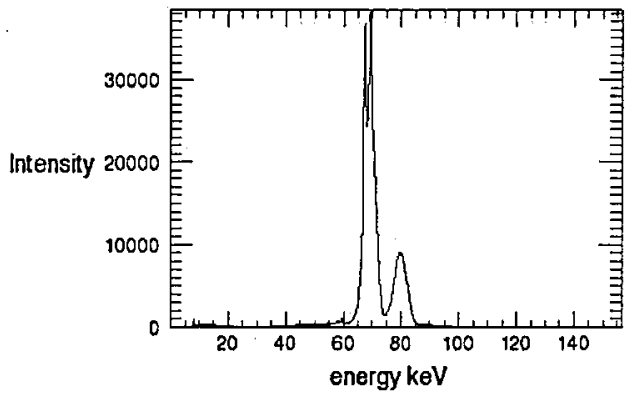

(c)

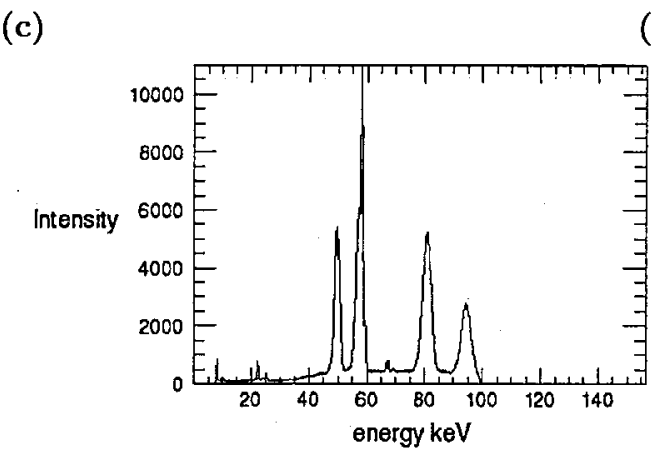

(e)

(d)
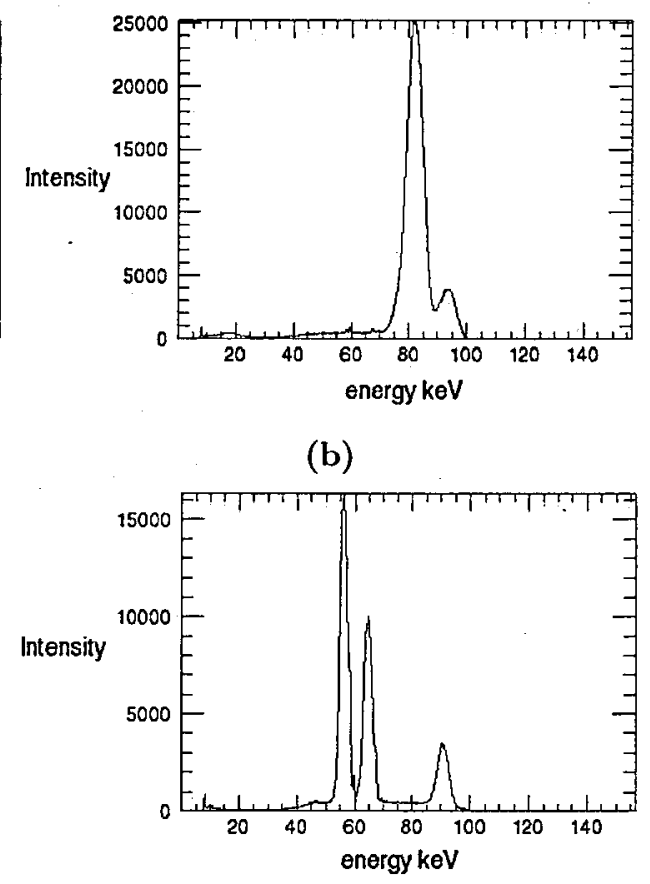

(b)

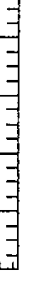

e)

Figure 4.9: Spectra obtained for $3 \mathrm{~mm}$ austenite-phase steel at scattering angles of; (a) $3^{\circ}$, (b) $4^{\circ}$, (c) $5^{\circ}$, (d) $6^{\circ}$ and (e) $7^{\circ}$. 


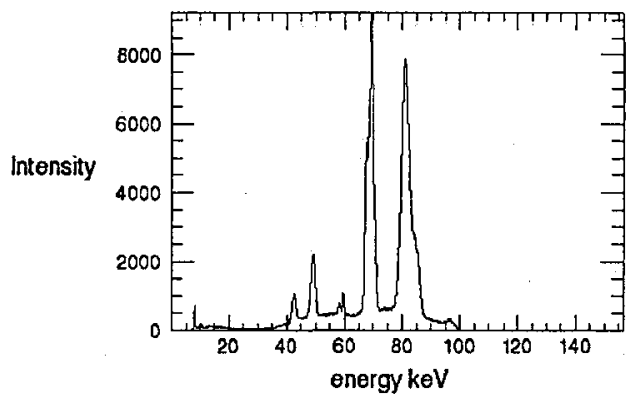

(a)

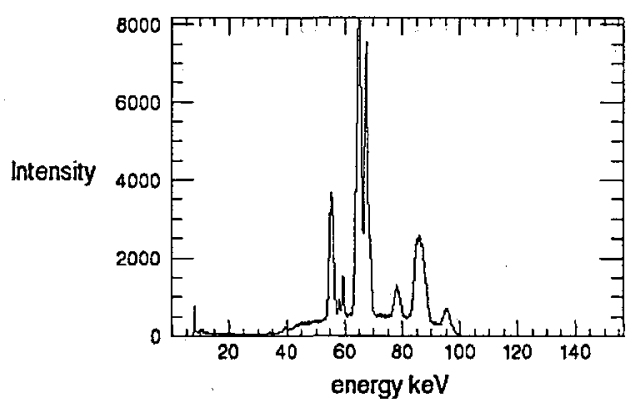

(c)

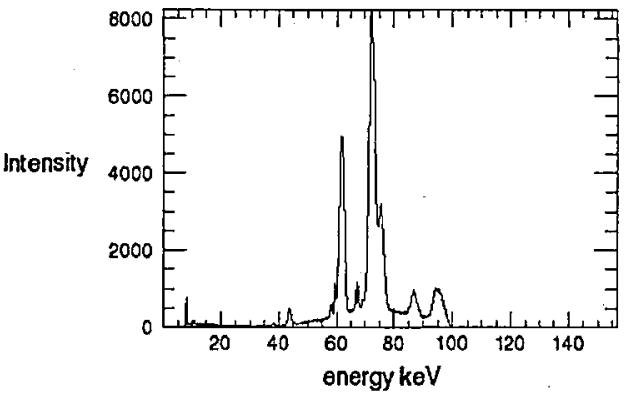

(b)

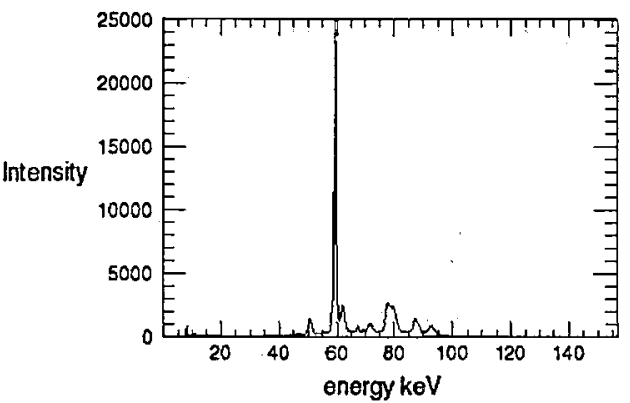

(d)

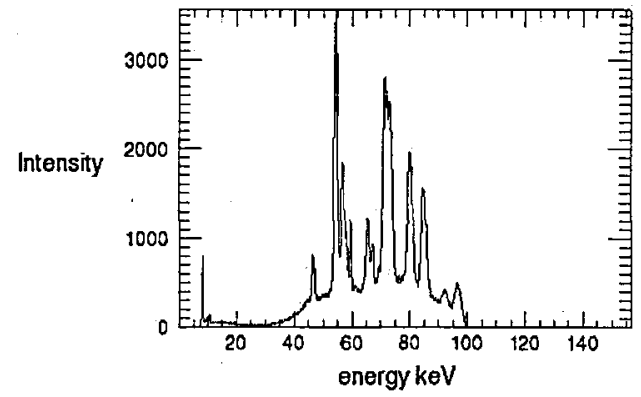

(e)

Figure 4.10: Spectra obtained for austenite-phase steel at scattering angles of; (a) $8^{\circ}$, (b) $9^{\circ}$, (c) $10^{\circ}$, (d) $11^{\circ}$ and (e) $12^{\circ}$. 


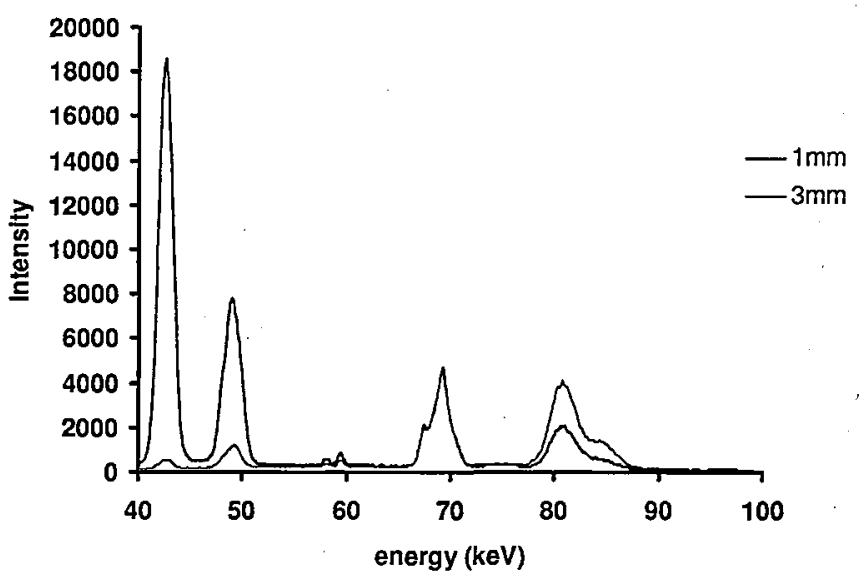

Figure 4.11: Differential attenuation becomes increasingly noticeable as the sample thickness increases from $1 \mathrm{~mm}$ to $3 \mathrm{~mm}$, with low energy photons from the $42 \mathrm{keV}$ and $49 \mathrm{keV}$ peaks at $8^{\circ}$ (corresponding to the $d_{111}$ and $d_{200}$ lattice spacings) being preferentially attenuated. The increase in the number of counts of the $80 \mathrm{keV}$ peak can be associated with an increase in the scattering volume (i.e. thicker sample).

results to within $0.4 \%$ [29].

As discussed, differential attenuation manifests itself as a significant effect once the sample thickness increases from $1 \mathrm{~mm}$ to $3 \mathrm{~mm}$. Although this does not affect the actual peak positions (i.e. lattice spacing measurements) it has a significant effect on the relative peak intensities, increasing the difficulty of distinguishing the lower energy peaks from the background. The difference between spectra collected at $8^{\circ}$ for $1 \mathrm{~mm}$ and $3 \mathrm{~mm}$ austenite-phase steel is shown in Fig. 4.11. While not significant in terms of measuring changes to samples of uniform thickness (with measurement of lattice spacings the requirement is simply for sufficient statistics to distinguish the peaks), in future measurements normalisation of the scattered beam to the measured 


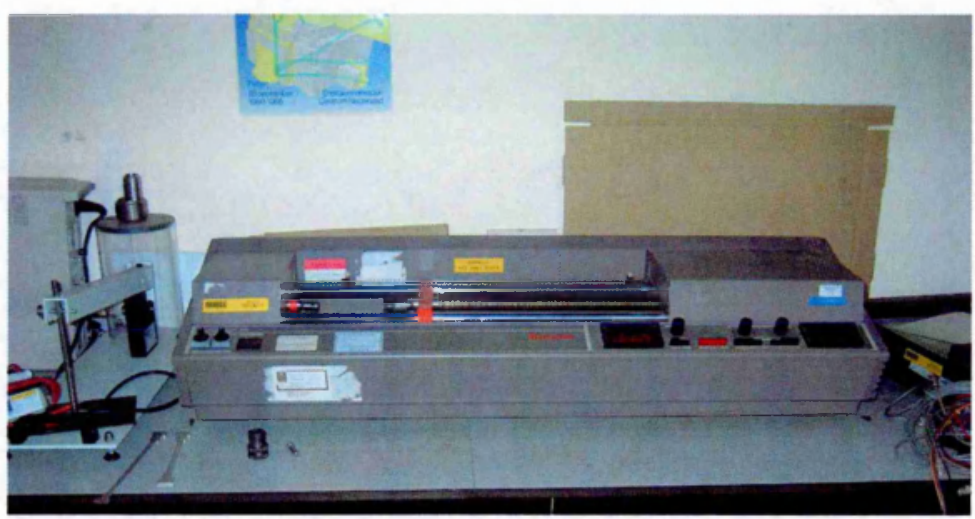

Figure 4.12: Photograph of one of the Monsanto tensometers at HMS Sultan's Nuclear Department. This particular apparatus was used to produce extreme plastic deformation in $3 \mathrm{~mm}$ thick austenitic stainless steel samples An identical apparatus was later incorporated into the EDXRD rig at Surrey. Visible in the lower left of the image are a pair of specifically designed steel samples used with this apparatus.

transmitted $\left(0^{\circ}\right)$ beam may be incorporated, should comparisons between different sample thicknesses be required.

A Monsanto tensometer, as shown in Fig. 4.12 (identical to the one later utilised, and damaged whilst performing dynamic stress measurements) was used to plastically deform austenitic stainless steel samples. The system, like its twin, is capable of applying a 20kN load, taking samples several millimetres thick up to the point of failure. In order to test the viability of the energy dispersive configuration to measure stress, a series of test austenitic stainless steel samples were provided by HMS Sultan. Unfortunately no details of the load applied in stressing these samples were provided, and so correlation could only be made between the lattice spacings and the measured 


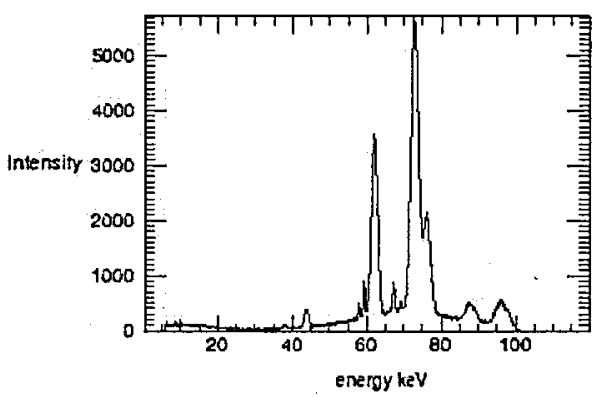

(a)

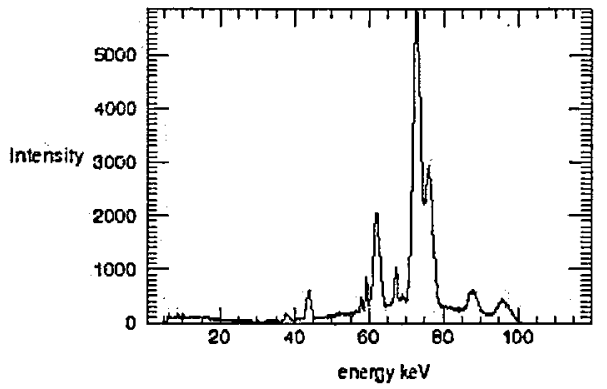

(c)

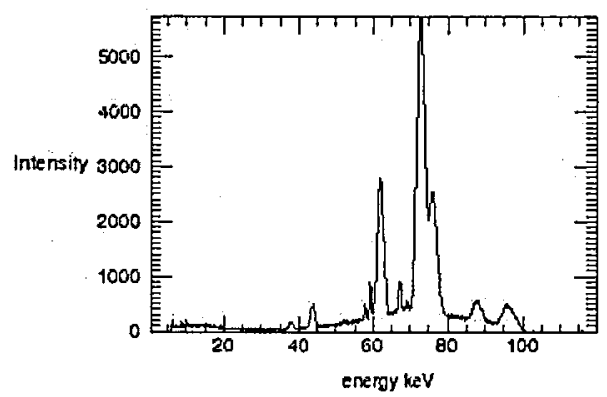

(b)

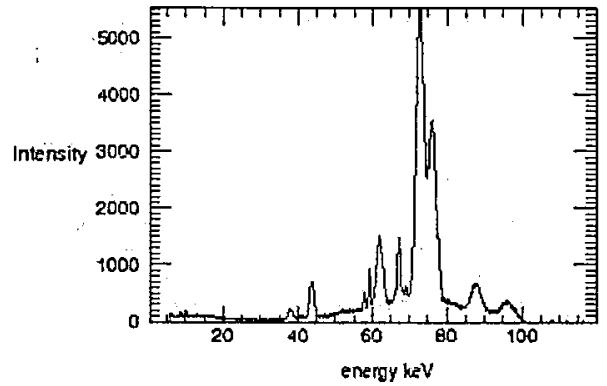

(d)

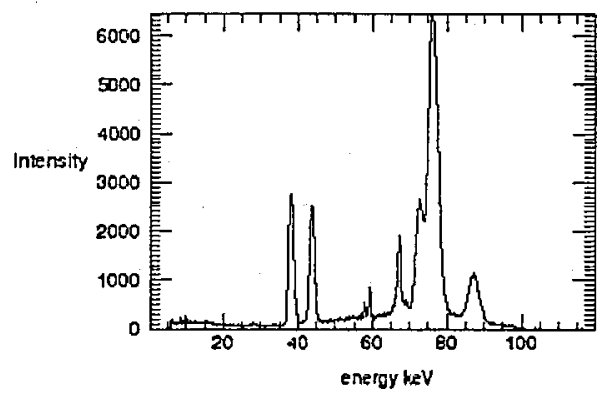

(e)

Figure 4.13: EDXRD spectra from samples of austenitic stainless steel of lengths (a) $19.7 \mathrm{~cm}$, (b) $20.1 \mathrm{~cm}$, (c) $20.7 \mathrm{~cm}$, (d) $21.4 \mathrm{~cm}$ and (e) $26.5 \mathrm{~cm}$. In all deformed cases the initial sample length was $19.7 \mathrm{~cm}$. Of particular interest is the $26.5 \mathrm{~cm}$ sample (refer to Fig. 4.15 and Fig. 4.17). 


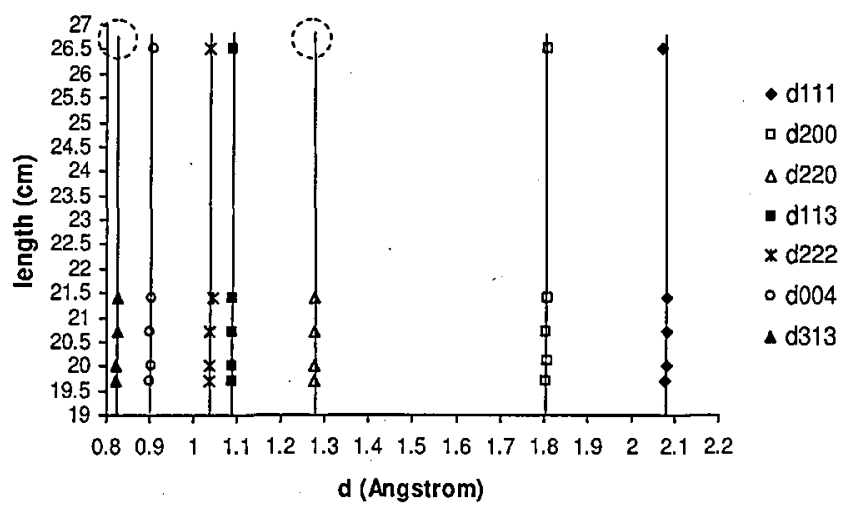

Figure 4.14: The lattice spacings for austenite samples of length from $19.7 \mathrm{~cm}$ to $26.5 \mathrm{~cm}$. There are no visible changes in lattice spacings, which corresponds to the crystal planes slipping past each other as the sample is plastically deformed. The key feature to note here however, is the missing lattice spacings for the $26.5 \mathrm{~cm}$ sample (indicated).

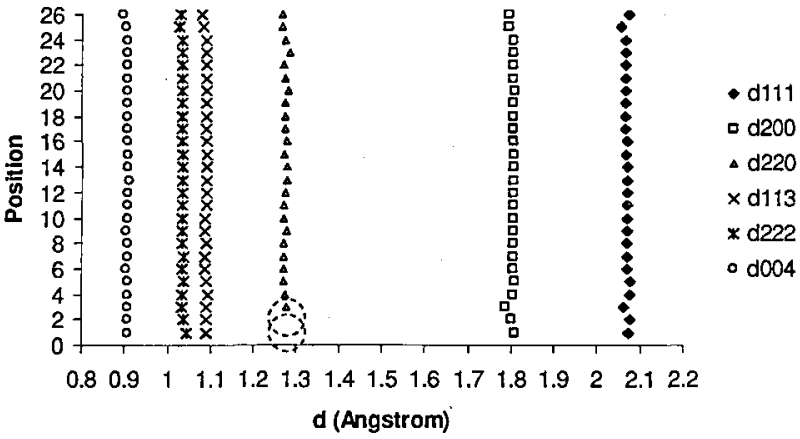

Figure 4.15: The longest of the samples has been sufficiently stressed to produce a 'necking point' where failure would occur should further loading be applied. Position 1 corresponds to this point while position 26 corresponds to the unstressed end of the sample. No significant changes to lattice spacing positions are visible, the result of slip deformation of the crystal structure. Again however, the striking feature here, is the lack of the $d_{220}$ spacing around the necking point. 


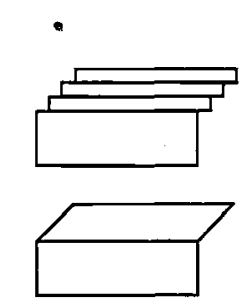

Macroscopic sheer due to many dislocations
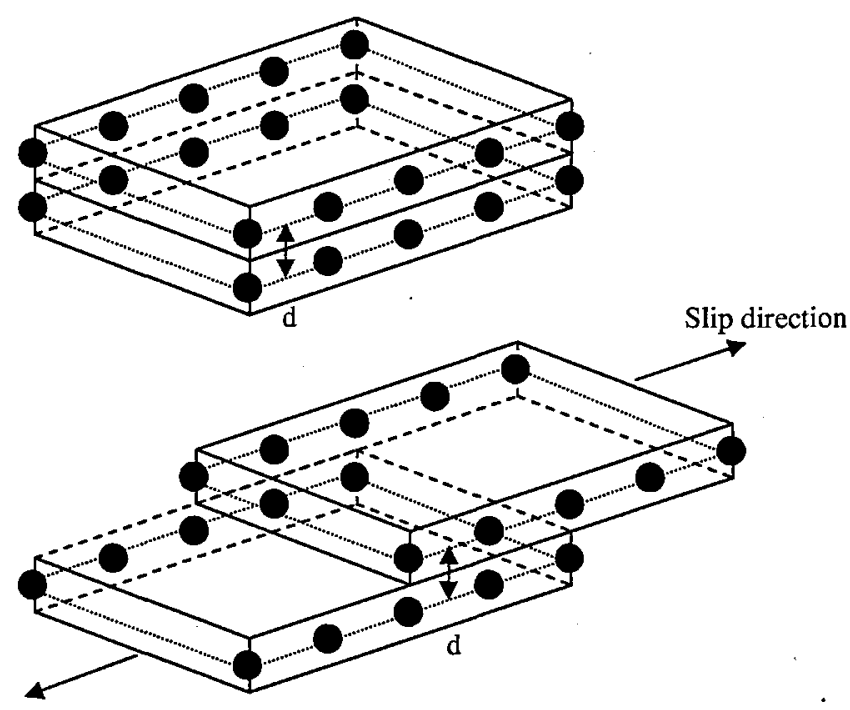

Figure 4.16: Slip is a plastic deformation of the material that involves displacement of one part of the crystal along with respect to an adjacent part. This occurs along a plane with small miller indices, i.e. the $\{111\}$ plane in FCC structures. The resulting.deformation does not alter the crystal structure or the lattice spacing $d$ indicated.

length of the samples. Samples of length $19.7 \mathrm{~cm}, 20.01 \mathrm{~cm}, 20.7 \mathrm{~cm}, 21.4 \mathrm{~cm}$ and $26.5 \mathrm{~cm}$ have been investigated using the system shown in Fig. 3.6, the results for which are shown in Fig. 4.13. Of particular interest is the $26.5 \mathrm{~cm}$ sample, given that this has been taken almost to the point of failure. Starting from a central point (where the onset of sample failure is evident), a series of measurements have been carried out along the length of the sample at intervals of $5 \mathrm{~mm}$.

No discernable changes in lattice spacings have been observed across the full range of samples, as summarised in Fig. 4.14 and Fig. 4.15. This correlates to slip deformation of the sample i.e. movement of the parallel crystal 
planes of the sample as it is stressed, without alteration to the crystal orientation or changes in the lattice spacings (refer to Fig. 4.16). What is noticeable however, is the change in relative intensities of the peaks as the sample length increases. Particularly noticeable is the change in the peaks corresponding to the $d_{220}(62 \mathrm{keV})$ and $d_{222}(77 \mathrm{keV})$ lattice spacings; the former decreasing in intensity while the latter increases in intensity with the increasing stress.

The most noticeable of these changes are visible in the appearance of the spectra for the $26.5 \mathrm{~cm}$ sample compared to all other samples, namely the loss of peaks corresponding to the $d_{313}$ and $d_{220}$ lattice spacings of austenite as shown in Fig. 4.17. This sample is the most acutely stressed, taken almost to the point of failure, where the loss of the associated austenite peaks is possibly the result of a stress induced phase transformation from the facecentred cubic to body-centred tetragonal structure (refer to Fig. 4.18), i.e. austenite to the martensite phase, which can occur at room temperature in this particular austenitic stainless steel (type 304L) with an applied strain [47][48] (refer also to section 4.3.3 with regards to further work on martensite transformations). The visible increase in the intensities of the $d_{111}$ and $d_{200}$ spacings may be partially attributable to reduced differential attenuation (the sample has thinned in this location), but are likely (particularly for the higher energy $d_{200}$ peak) indicative of the structural changes occurring in this location.

In order to ensure the observed changes seen here are not simply the result of texturing effects (and a preferred orientation of the crystalline structure) the sample has been rotated through a total of $360^{\circ}$, with EDXRD measurements taken at intermediate angles. In addition to this the samples have 


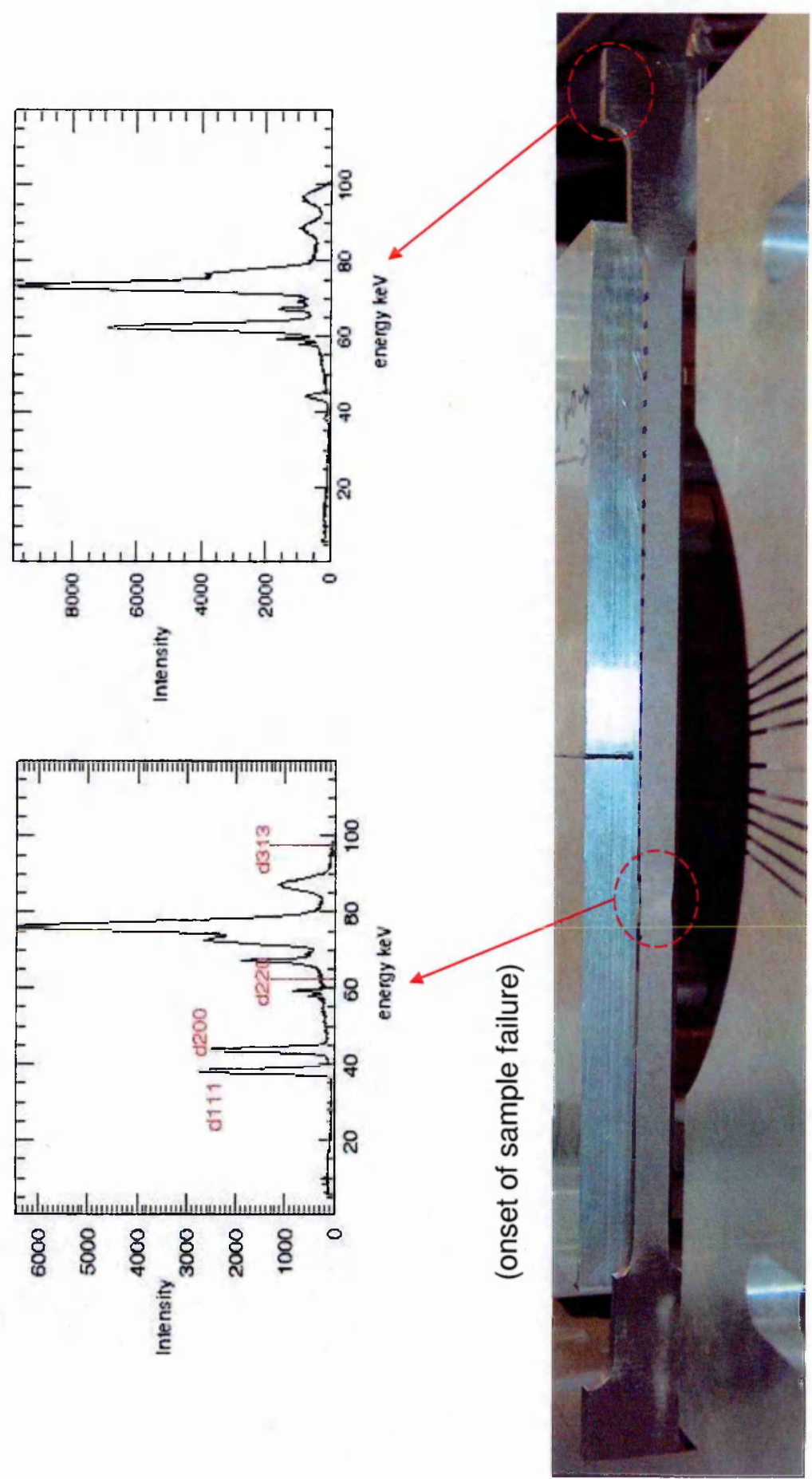

Figure 4.17: Spectra obtained for a $26.5 \mathrm{~cm}$ long sample of austenite-phase steel. The spectrum to the right corresponds to the unstressed end of the sample, while the spectrum to the left corresponds to the 'necking point' where the sample is about to fail. The sudden loss of the austenite $d_{313}$ and $d_{220}$ lattice spacings is possibly the result of a phase change induced by the stressing. 


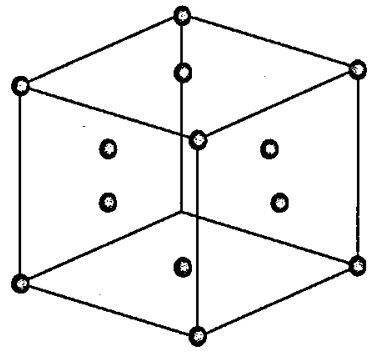

$\propto$ FCC structure

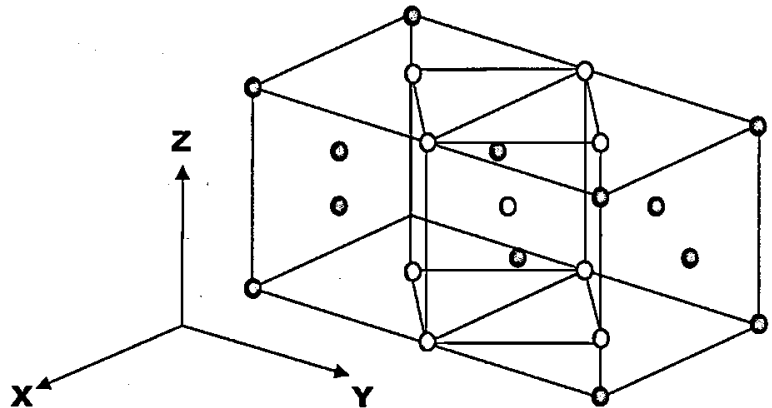

$\propto$ BCT structure

Figure 4.18: Austenite-phase steel is formed of a face-centred cubic (FCC) structure. Should two FCC crystals be positioned side by side another structure; body-centred tetragonal (BCT) becomes visible. A uniform expansion along the $\mathrm{x}$ and $\mathrm{y}$ directions, combined with a compression along $\mathrm{z}$ would convert this into a BCC structure. Carbon interstituals however, prevent this becoming pure BCC. The resultant martensite is therefore a BCT structure, dependant on the total carbon content of the steel.

been cut and imaged using HMS Sultan's SEM in order observe the grain structure as shown in Fig. 4.19.

The measurements described above utilised a delicate thin-windowed HPGe detector that requires constant liquid nitrogen cooling to $77 \mathrm{~K}$. While such a device is perfectly acceptable in a laboratory environment, it would not be practicable in any form of field deployable application. The Amptek XR100T CZT detector introduced in section 3.4.2 however, is a more robust, 


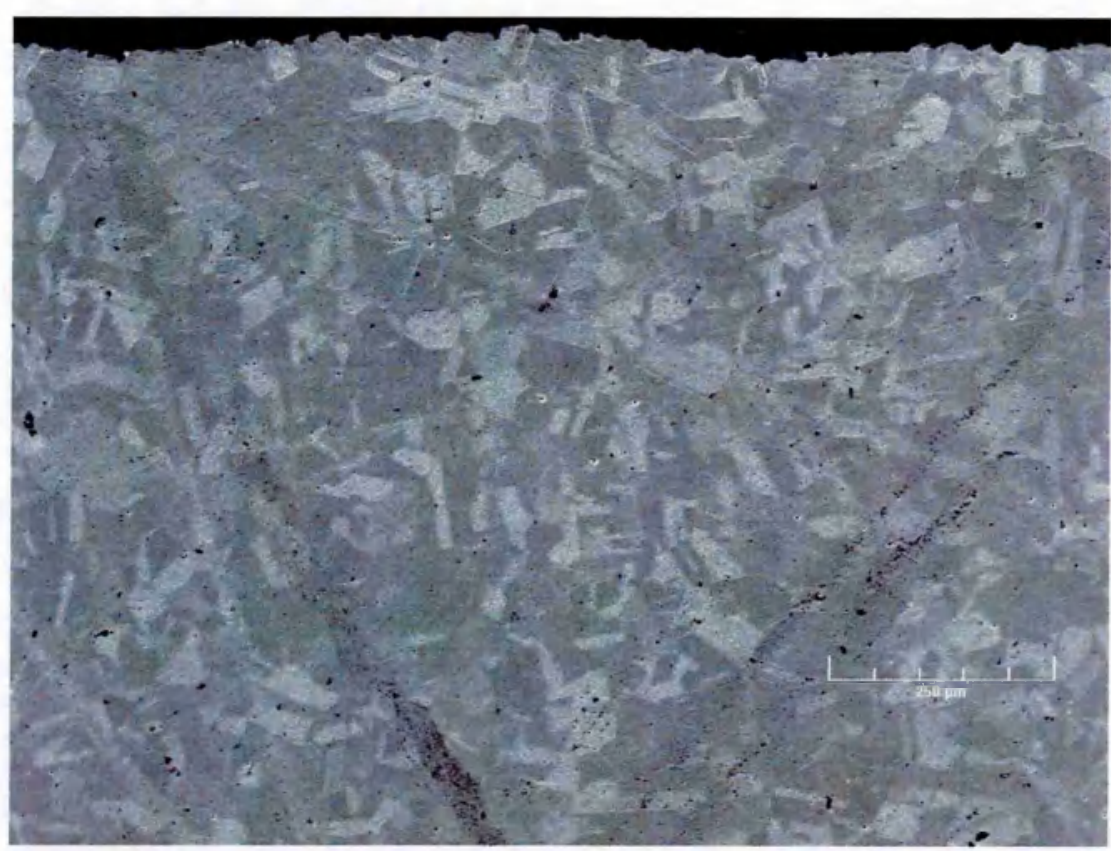

(a)

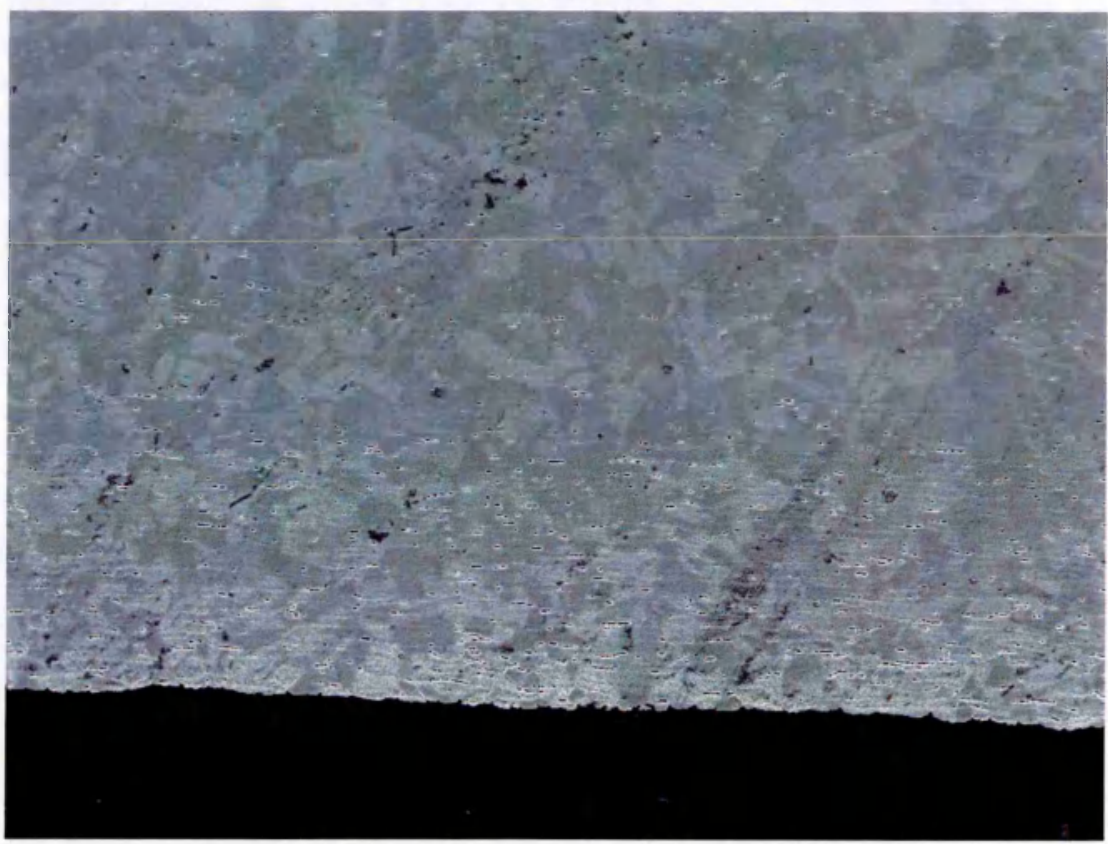

(b)

Figure 4.19: SEM images showing the grain structure at the (a) outer, and (b) inner edges of a plastically deformed 304L sample (courtesy of S. E. Jarmen, HMS Sultan) [49]. While there are some near-surface artefacts caused by stress in (b), there are no significant changes to the grain structure. X-ray diffraction measurements with the sample at different angles with respect to the beam yielded no changes associated with preferred orientation. 


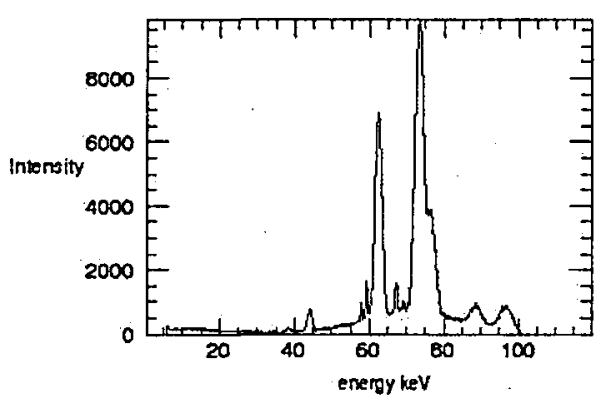

(a)

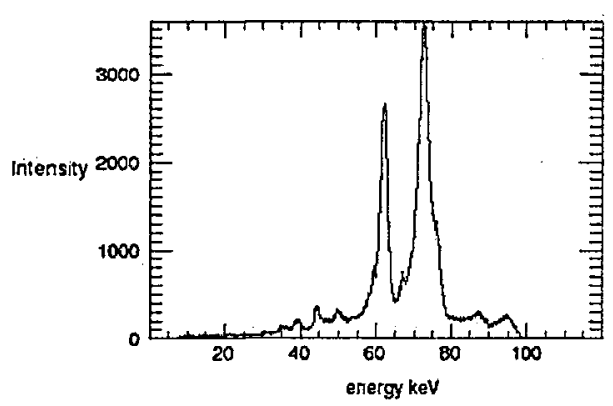

(c)

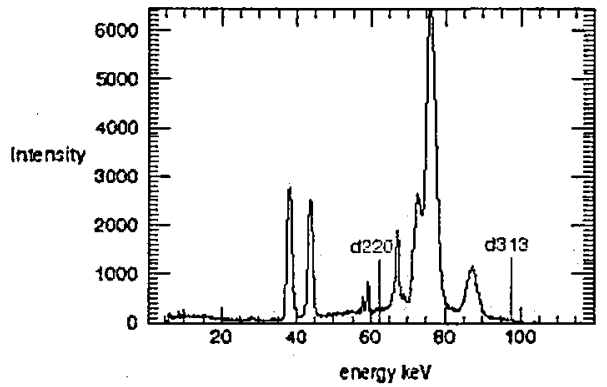

(b)

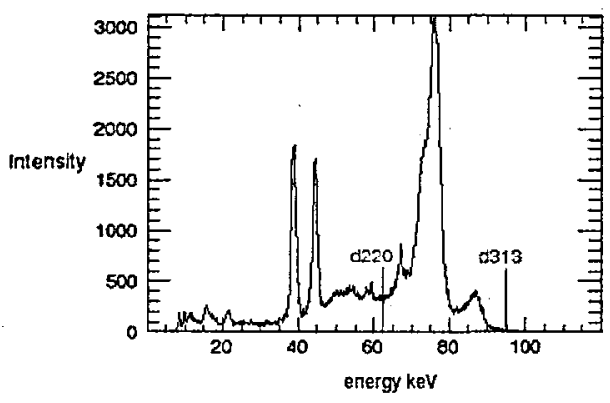

(d)

Figure 4.20: Comparison of the data obtained for an austenite-phase sample that has undergone extreme plastic deformation; with the Ortec HPGe detector (a) and (b), and the compact Amptek XR-100T device (c) and (d). As clearly indicated, despite the lower energy resolution, the CZT device is more than capable of imaging the structural changes observed in the sample. 
electrically cooled device. This detector has been used in parallel with the Ortec HPGe for investigating the EDXRD spectra obtained from the plastically deformed austenite-phase samples [50]. As can be seen in Fig. 4.20 despite the reduced energy resolution of the CZT, the system is more than capable of imaging the changes introduced as the austenite sample is taken up to the point of failure.

For possible future investigations using this apparatus (including stress corrosion cracking in samples of interest to NNPP) refer to section 5.3.

\subsubsection{Martensite phase transformation}

The comparison between samples of ferrite-phase (body-centred cubic) and austenite-phase (face-centred cubic) steel demonstrates the significant differences between the spectra of the different phases. Previous work published in Nucl. Instr. and Meth B and involving angular dispersive XRD [36] demonstrated the feasibility of measuring the phase transformation from the ferrite to austenite phase of steel that would occur through the annealing process in a online steel mill. While the ferrite to austenite transformation produces a steel with the desired properties for an extensive array of applications including the Nuclear industry, there is another phase transformation that is desired, namely that of austenite to martensite.

While austenite-phase steel has high strength and ductility, its mechanical properties may be enhanced through inducing a partial change to the martensite phase, greatly improving the toughness of the steel. As discussed previously the martensite phase may be induced through plastic strain and deformation of austenite steels (such as 304L), that often behave as metastable 
alloys, allowing for the formation of the second phase. Martensite formation may also be spontaneously induced through rapid cooling; quenching of austenite-phase steels has been a widely used process for centuries, for example, in the manufacture of swords, where the martensite transformation is desired for the production of a hard and therefore, exceptionally sharp blade [51]. Martensite formation requires a nucleation site, and in the case of plastic strain the nucleation sites are along the slip bands produced as the sample is deformed. Residual stresses in an austenite sample provide the nucleation sites for spontaneous production of martensite through cooling, with martensite plates formed inside (and confined by) the austenite grain boundaries [52]. This process induces a compressive stress on the austenite grains leading to an overall reduction in residual stress of the material and therefore reduced susceptibility, for example, to stress corrosion cracking.

The latter process of martensite formation has been investigated using energy dispersive XRD. A series of $3 \mathrm{~mm}$ samples of austenitic stainless steel have been provided by the Nuclear Department at HMS Sultan. These samples are formed in near-circular or elliptical shaped rings (depending on the level of compressive loading applied) with axis of dimensions $a=4.0 \mathrm{~cm}$ and $b=2.6 \mathrm{~cm}$ for the extremely plastically deformed elliptical sample, such that residual stresses will be present. This has been modelled using the Abaqus Finite Element (FE) model analysis software (SIMULIA, Dassault Systemes, USA) [53] as indicated in Fig. 4.21, the areas of plastic strain should act as nucleation sites for martensite production given sufficient cryogenic cooling. In terms of the X-ray scattering profiles generated, a decrease in the intensity of the characteristic FCC-structured austenite peaks would be expected 


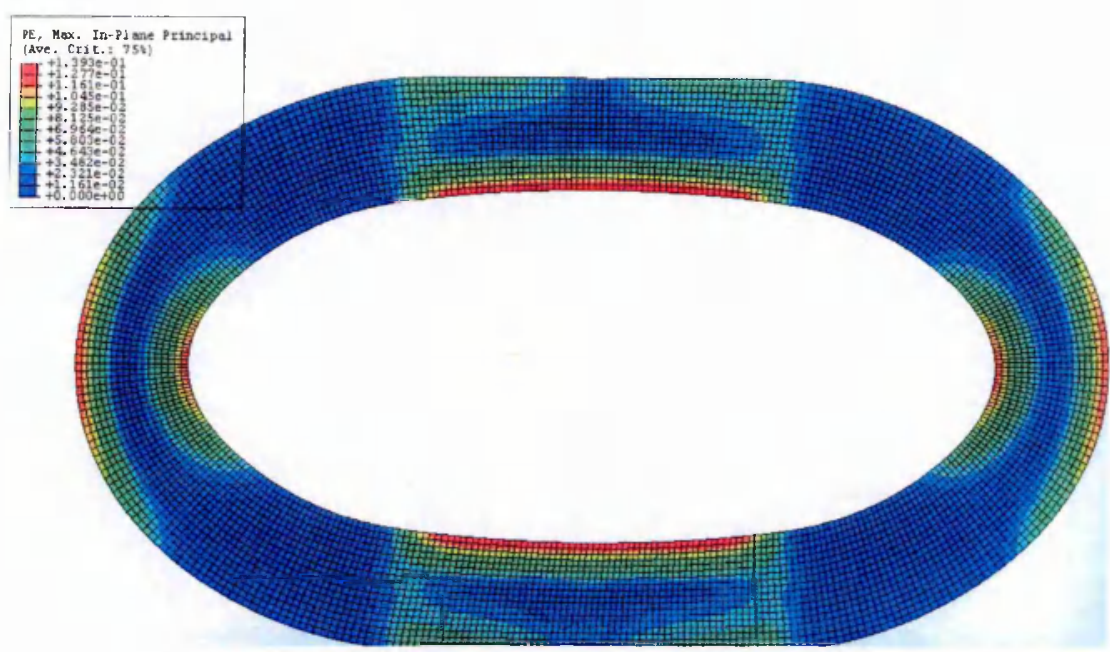

Figure 4.21: Abaqus finite element modelling of the residual stresses and plastic strains in a $3 \mathrm{~mm}$ thick (axis of length $4 \mathrm{~cm}$ and $2.6 \mathrm{~cm}$ ) austenitic stainless steel sample [54]. A similar, lesser deformed sample has been cryogenically cooled to induce the martensite phase transformation, with transmission XRD measurements used to map the concentrations of martensite in the bulk of the sample.

in addition to increasing intensity of the peaks corresponding to the lattice spacings of the BCT-structured martensite.

Further finite element modelling at HMS Sultan suggested that a lesser deformed, near-circular sample would have a larger residual stress field than the extremely deformed, oval shaped sample. This particular sample has been mapped with EDXRD both before and after quenching in liquid nitrogen, using the symmetry of the sample to reduce the number of data points required. Total acquisition time was set at 10 minutes per measurement point, utilising the full flux of the $225 \mathrm{kV}$ tube at $100 \mathrm{kV}$ and a $30 \mathrm{~mA}$ beam current. Beam geometry consisted of the same $1 \mathrm{~mm}$ by $10 \mathrm{~mm}$ ribbon beam 
that has been previously used for EDXRD work. As indicated in Fig. 4.22, each map consists of a pair of line scans across one quadrant of the sample, encompassing the areas of high/low plastic strain indicated in the finite element model. On each line scan, measurements are taken at $1 \mathrm{~mm}$ intervals horizontally, whilst the line scans themselves are separated by $10 \mathrm{~mm}$ vertically for a total of 26 measurement points per map. The position of the measurement points is correlated based upon the reported stepper motor positions, so that the same points may be investigated both prior to, and after quenching. The starting position of $+20,000 /+1000$ is indicated, and chosen so that the beam does not interact with the clamps used to hold the sample in place. Whilst the stepper-motor system is designed to provide consistent movements and therefore, reproducible measurement points, this is further verified by the physical edge of the sample i.e. continuation of the line scans until the diffraction peaks disappear completely, giving a physical 'end-point' that the relative motor positions can be related to.

In Fig. 4.23 several of the same data points, post quenching are displayed. What is immediately striking is the large differences in relative intensities of the $d_{111}, d_{200}$ and $d_{220}$ peaks at motor position $17,600 /-9000$ corresponding to the inside edge of the sample. Of particular note is the sudden, sharp increase in the $d_{200}$ peak $(40 \mathrm{keV})$, combined with the sudden suppression of the $d_{220}$ peak (at $62 \mathrm{keV}$ ) which matches the changes seen in the extreme plastic deformation samples used in section 4.3.2, where martensite formation is thought to occur. In this case the $17,600 /-9000$ point after quenching shows the same structural changes as the highly deformed 'necking' point in the $26.5 \mathrm{~cm}$ austenite-phase sample. The changes are not limited to this 


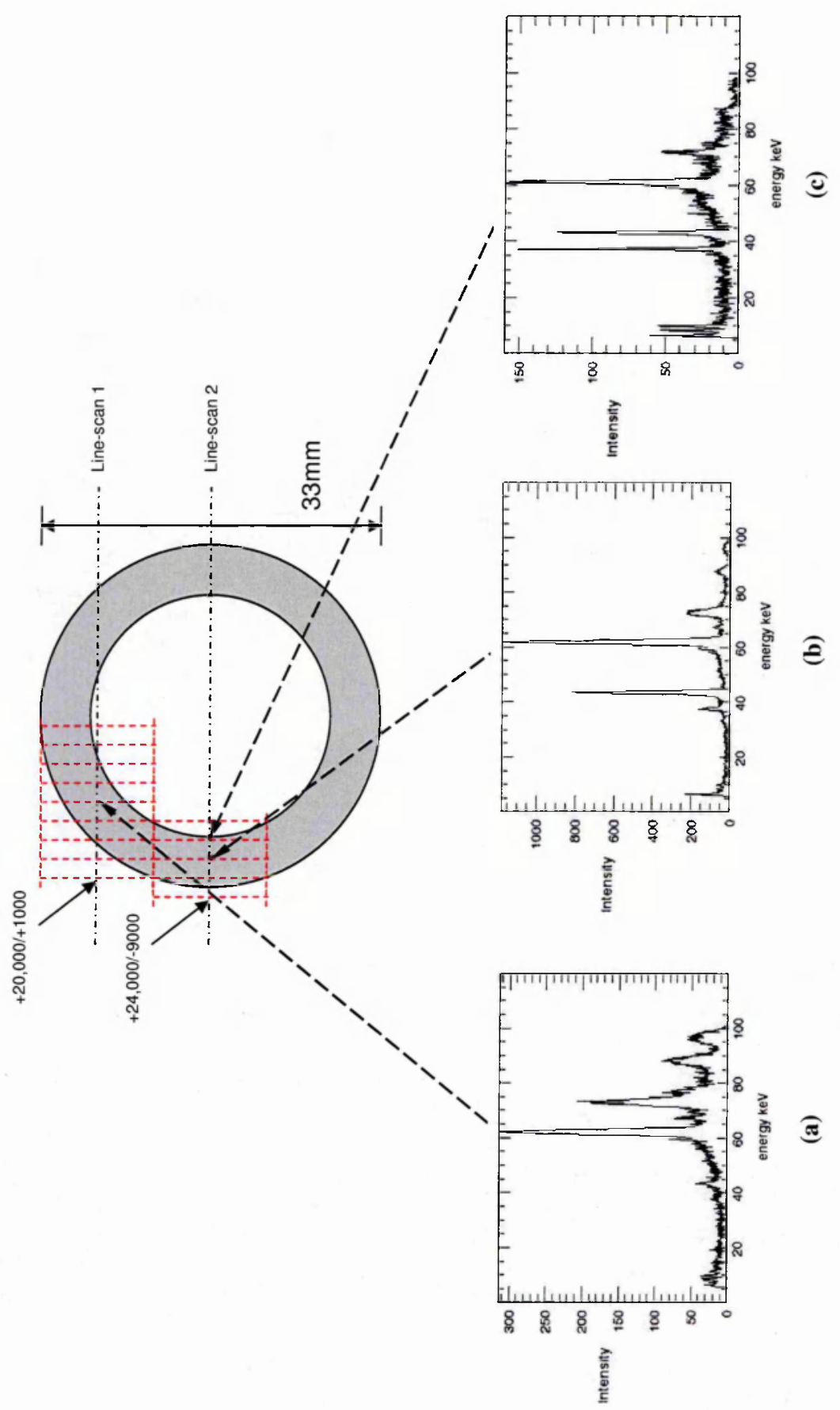

Figure 4.22: Mapping of the austenite-phase steel ring prior to quenching. The spectra shown represent areas of minimal plastic strain (a), through to areas of increasing plastic strain $(b, c)$. 


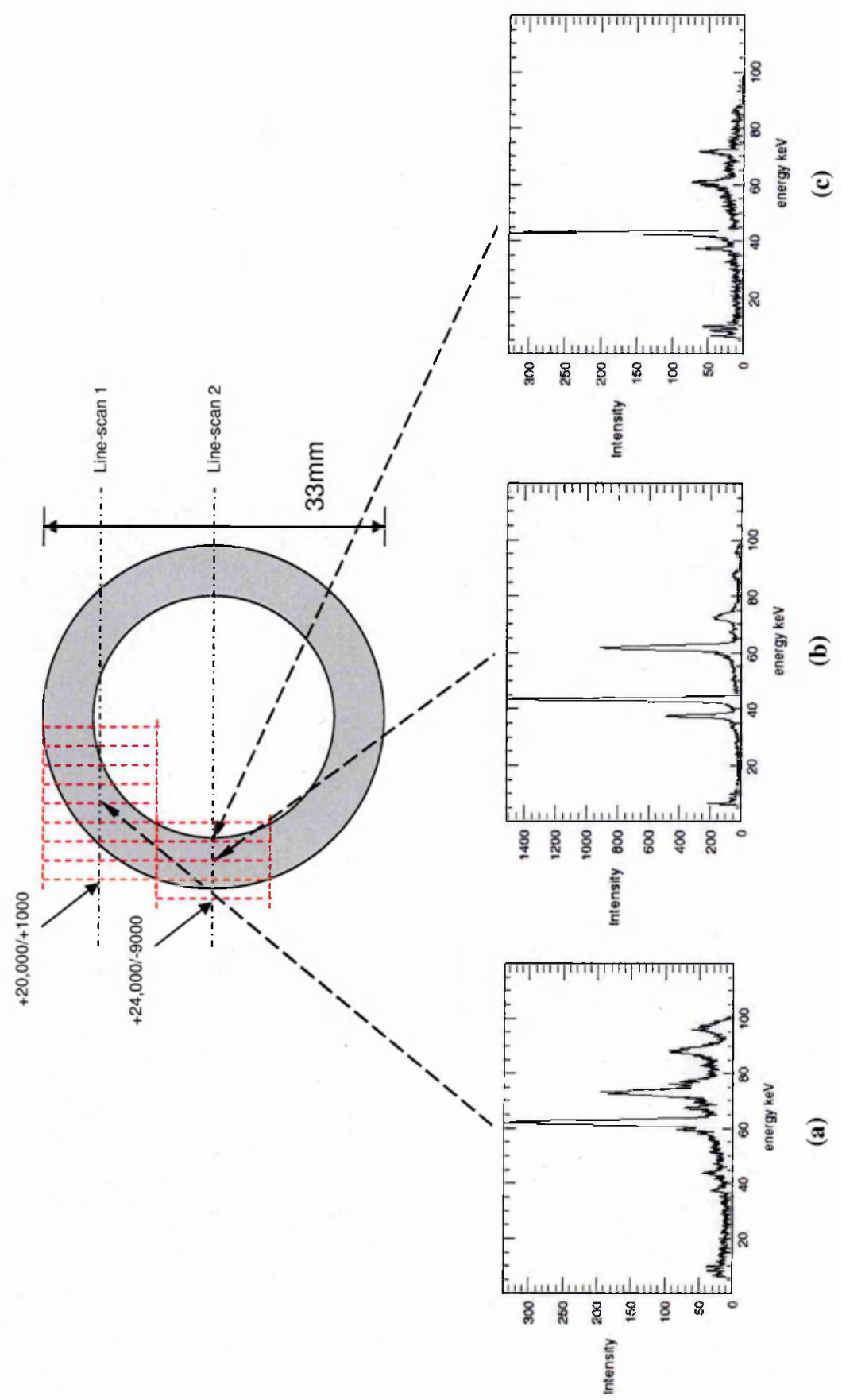

Figure 4.23: Follow up map after quenching. Compared to the spectra in Fig. 4.22, the region corresponding to minimal plastic strain is unchanged. However, spectra corresponding to the areas of increasing plastic strain demonstrate noticeable changes in the intensities of the $d_{111}$ and more significantly, $d_{200}$ $(40 \mathrm{keV})$ and $d_{220}(62 \mathrm{keV})$ peaks. 


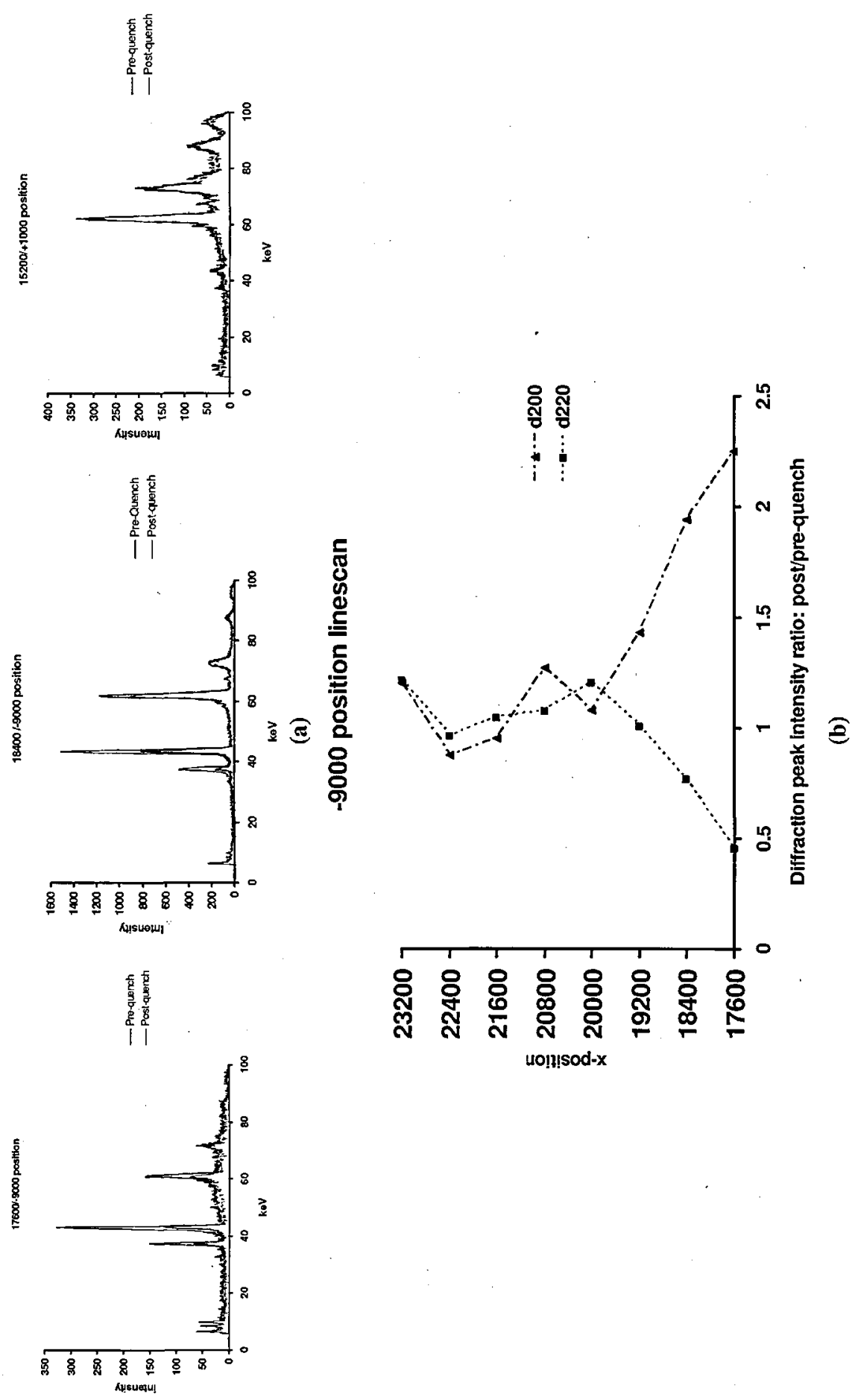

Figure 4.24: Comparisons of key lattice spacing intensities pre and postquenching. (a) The spectra obtained at the 17,600/-9000, 18,400/-9000 and $15,200 / 1000$ points after cooling in nitrogen, are superimposed with those spectra obtained prior to quenching. (b) The ratio of the $d_{200}$ and $d_{220}$ spacings before and after quenching highlights the striking difference around the 17,600/-9000 point, corresponding to an area of high plastic strain. 
single data point; comparing the 18,400/-9000 and 19,200/-9000 data points before and after quenching shows a subtle fall-off in the differences in relative intensities of the peaks related to the d-spacings in question, pre and post quenching, which would follow the expected fall-off in residual stress field away from the inner edge.

This effect is illustrated more clearly in Fig. 4.24, where the difference in the ratio of the intensities of the $d_{200}$ and $d_{220}$ spacings before, and after quenching, is plotted against position for the -9000 line scan.

Given that martensite forms along the areas of plastic strain, and that the fall-off matches the predictions, this validates the finite element code used to generate the map of Fig. 4.21, which may be used to predict stresses and plastic strains in further specimens of interest to the NNPP.

\subsubsection{Dynamic stress measurements using EDXRD}

Following on from the static measurements using plastically deformed samples the Monsanto tensometer was incorporated into the X-ray diffraction apparatus as described previously in section 3.2.1. The samples are designed such that tensile loading is uniform across the full length of the sample; in terms of X-ray diffraction concerns it is sufficient to simply ensure the scattered and incident beams are aligned so that the scattering voxel is centred in the bulk of the sample. The HPGe detector was once again placed at a fixed scattering angle of $9^{\circ}$ and its energy scale pre-calibrated using a variable $\mathrm{X}$-ray source. Once again, the $\mathrm{X}$-ray tube was limited to $100 \mathrm{kV} / 10 \mathrm{~mA}$ throughout the experiment.

The first sample was taken from $0 \mathrm{kN}$ up to a $7.5 \mathrm{kN}$ load, i.e. a yield 


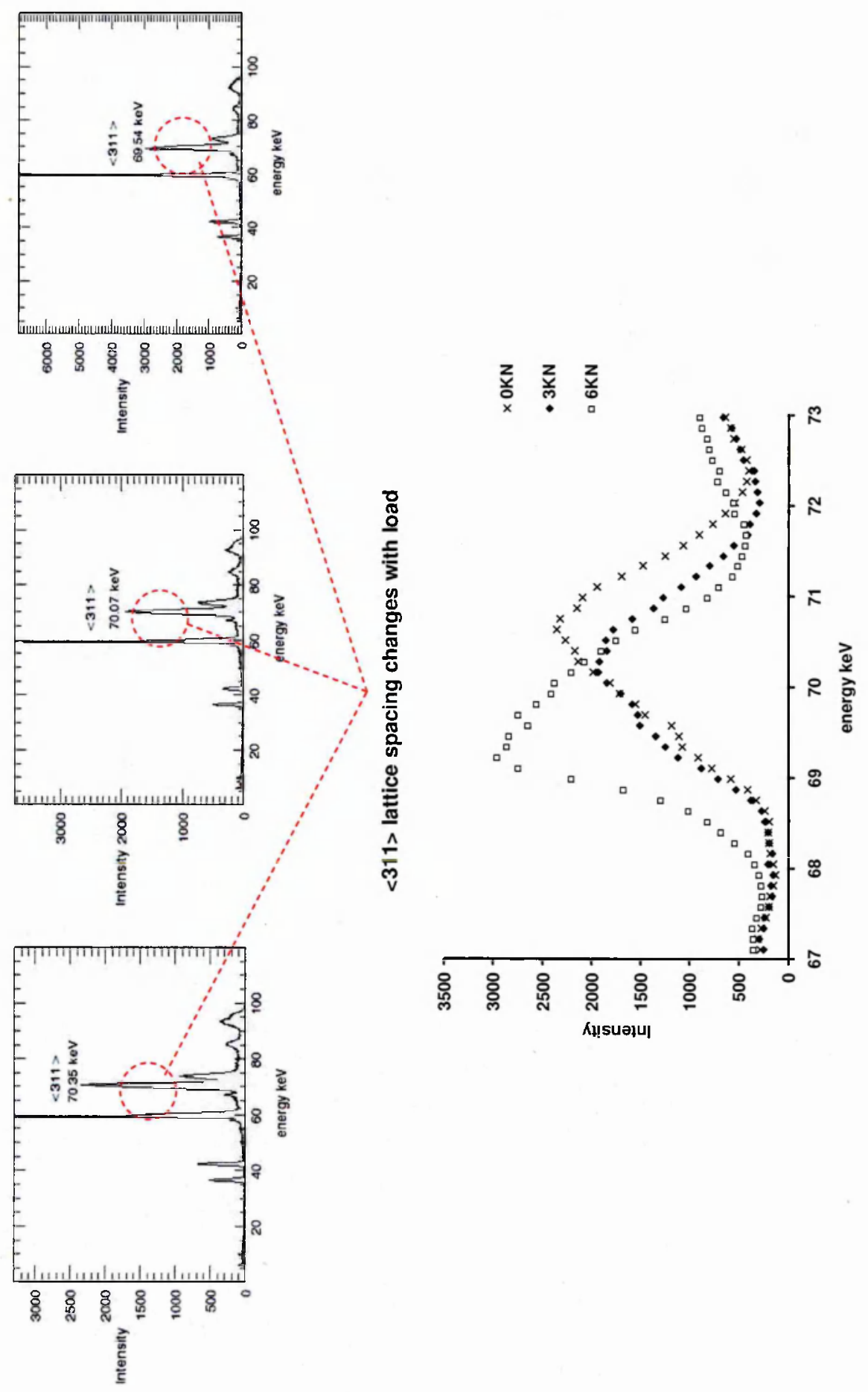

Figure 4.25: The results of EDXRD on steel undergoing tensile loading. The $d_{311}$ spacing is the key lattice spacing of interest given that it should demonstrate the most linear response to strain. As is clearly visible here, the increase in dspacing due to the increasing applied load is shown by the shift in the energy of the $d_{311}$ peak. 
stress of $0 \mathrm{MPa}$ through to $330 \mathrm{MPa}$, the latter being the approximate point where plastic deformation occurs. In Fig. 4.25 the EDXRD spectra obtained at yield stresses of $0 \mathrm{MPa}, 143 \mathrm{MPa}$ and $286 \mathrm{MPa}$ are displayed. For this particular alloy, the $d_{311}$ lattice spacing exhibits the most linear response to strain [55]. From the powder diffraction data [29] this corresponds to a dspacing of $1.07549 \AA$, and at a scattering angle of $9^{\circ}$ this particular spacing corresponds to an energy of $70 \mathrm{keV}$. Overlaying the spectra in the energy range $67 \mathrm{keV}$ to $73 \mathrm{keV}$ suitably demonstrates the subtle shift in $d_{311}$ peak position to lower energies. Whilst the asymmetry and width of the $d_{311}$ peak in these spectra precludes accurate measurement of the actual lattice spacing (compared to for example, powder diffraction data), it is important to note the relative shift is clear and approximately linear with loading as expected, with an approximate $0.5 \mathrm{keV}$ shift between measurements that corresponds to a $0.008 \AA$ increase in the $d_{311} \mathrm{~d}$-spacing, i.e. little more than $0.75 \%$.

Initially several more measurements were planned, unfortunately however, these were cancelled due to severe damage to the tensometer caused by malfunction. However, while in this particular work no further measurements were undertaken, it is important to note a possible avenue for improvement that was looked into prior to the equipment failure (and could be incorporated into any future work).

The raw transmission XRD data for the austenite-phase steel on this apparatus was reviewed for the full range of scattering angles available. For the $225 \mathrm{kV}$ tube operating at $100 \mathrm{kV}$, the bremsstrahlung output yields scattered photons from approximately $20 \mathrm{keV}$ to the end point at $100 \mathrm{keV}$. While a scattering angle of $9^{\circ}$ had, in general, proved optimal across the full range 


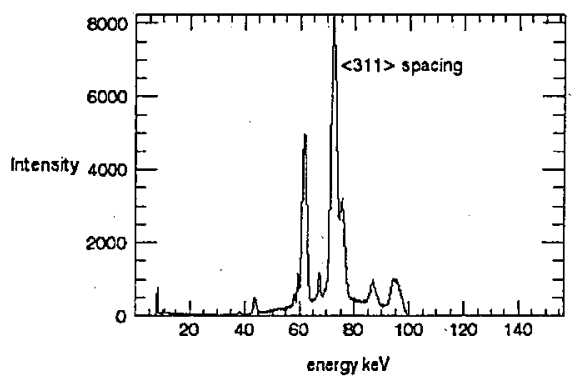

(a)

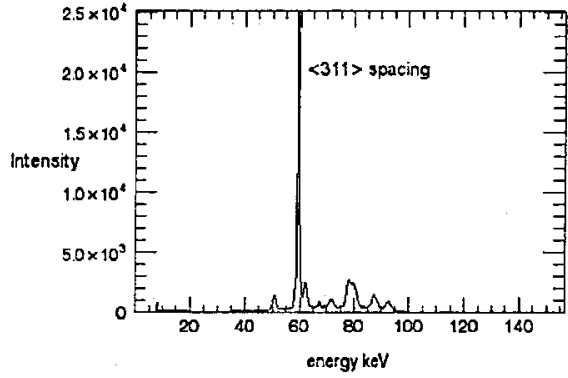

(b)

Figure 4.26: Comparison of the elastic scattering spectra obtained from $304 \mathrm{~L}$ steel at angles of (a) $9^{\circ}$ and (b) $11^{\circ}$, reveal the improvement in resolution of the $d_{311}$ peak at the higher angle.

of lattice spacings (corresponding to a momentum transfer $x=0.13 \AA^{-1}$ to $x=0.63 \AA^{-1}$ ), only the $d_{311}$ is of interest for dynamic measurements. As demonstrated in Fig. 4.26 increasing the scattering angle from $9^{\circ}$ to $11^{\circ}$ (shifting the corresponding momentum transfer range from $x=0.15 \AA^{-1}$ to $x=0.77 \AA^{-1}$ ) yields a significant improvement in the intensity and resolution of the $d_{311}$ peak for this sample.

\subsection{High energy diffraction}

The X-ray diffraction work described thus far is ultimately limited by the available flux of a bench-top system built around an industrial X-ray tube. In December 2006 a transmission X-ray diffraction experiment, designed by A. Price and M. Wenman .[56] (HMS Sultan), to which the author collaborated with, was carried out at the European Synchrotron Radiation Facility (ESRF) in Grenoble. This experiment involves the same principles as a 
bench-top system and proves a useful comparison of the capabilities of the system developed at Surrey and HMS Sultan to that of a cutting edge beamline at a large synchrotron.

The experiment was designed to investigate the effects of fatigue crack growth on the residual stress field in samples of the same low-alloy ferritic steel as described in the previous sections. The aim here was to validate the Abaqus finite element code used to model these stresses and ultimately to further understand how fatigue crack growth in the residual stress field induced during manufacture of the steel, affect the operating lifetime of the component. The ID15 beamline at the ESRF is designed specifically for high energy diffraction, with a polychromatic beam of up to $300 \mathrm{keV}$ collimated to $150 \mu \mathrm{m}$ by $150 \mu \mathrm{m}$. This in itself does not represent the limit of the systems capabilities, indeed, transmission-geometry diffraction in up to $8 \mathrm{~cm}$ of steel has been achieved with this beamline.

In order to generate the residual stress field, the samples (visible in Fig. 4.27) used were $25 \mathrm{~mm}$ thick, compact tension (CT) specimens with a notch cut into one side. Upon application of a $90 \mathrm{kN}$ compressive load either side of the notch, a residual stress field is generated. In the second specimen a $3 \mathrm{~mm}$ long fatigue crack was grown from the tip of the notch into the bulk of the material.

In the beamline itself, the experiment shares the same principles as the simple table-top system, HPGe detectors located at scattering angles of $5^{\circ}$ (horizontal and vertically) collect energy spectra which can then be correlated to lattice spacing. The scattering angle and high energy is such that unlike the bench-top system where the first measurable lattice spacing peak 


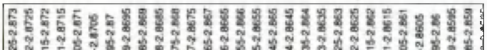

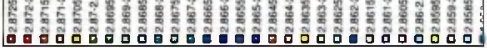
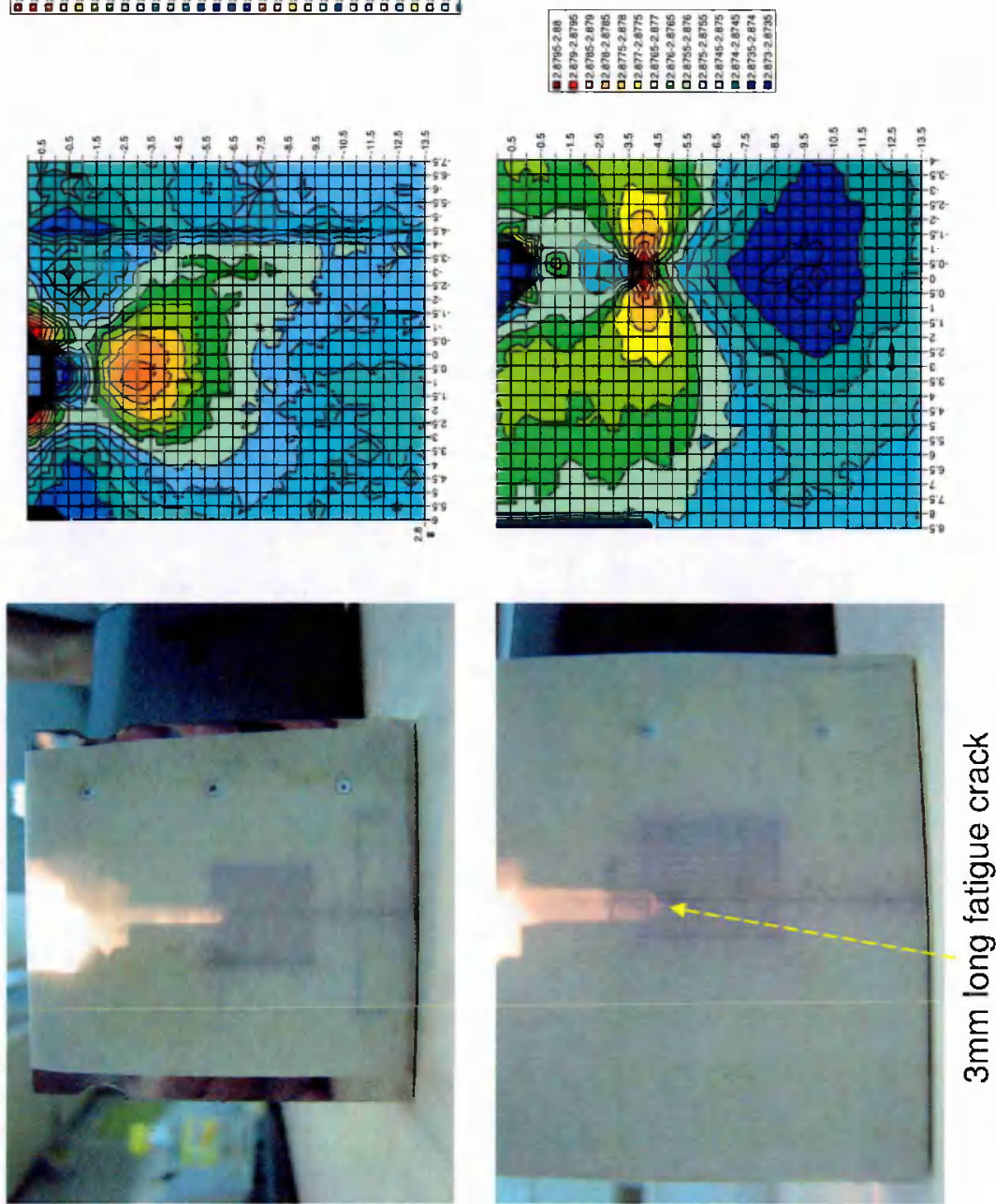

Figure 4.27: Photographs of the CT specimens and residual stress map obtained at the ID15A beamline at the ESRF. The outline of the X-ray diffraction map taken is visible on the $\mathrm{X}$-ray sensitive paper that was affixed prior to irradiation, while the position of the notch is clear from the illumination. Both specimens have been subjected to the same compressive load introducing the residual stress distribution shown in the top map (in terms of the changes to the cubic lattice parameter). The second sample has a $3 \mathrm{~mm}$ fatigue crack, producing the relaxation of the residual stress field behind the crack tip as shown in the bottom map [56]. 


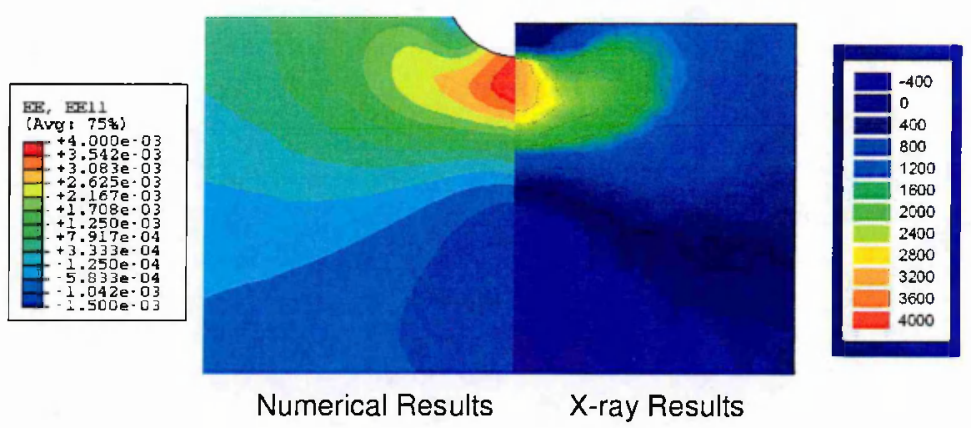

Figure 4.28: Abaqus finite element modelling of the residual stress field around the notch of the CT specimen compared to the data obtained at the ESRF, demonstrating the ability of the model to correctly predict the residual stress field. Courtesy of A. Price, HMS Sultan [57].

occurs at approximately $20 \mathrm{keV}$, here the lowest energy lattice spacing peak is found above the lead fluorescence peak at $75 \mathrm{keV}$. The chief advantage, aside from the penetration depth, is the lack of perceptible differential attenuation effects in samples of this thickness.

Mapping of the residual stress field was carried out by measuring the relative change in the cubic lattice parameter across a $15 \mathrm{~mm}$ by $15 \mathrm{~mm}$ map. The flux on the beamline is such that, even with $25 \mathrm{~mm}$ of steel to penetrate, diffraction measurements with powder diffraction quality resolution are achieved in 60 seconds. The initial maps are also shown in Fig. 4.27, clearly demonstrating how the fatigue crack releases the stress field as it grows through the material. As shown in Fig. 4.28, it is clear that the data obtained verifies the finite element model used, and future ESRF experiments may be used to further develop the model with regards to crack growth in the same ring-specimens used on the bench-top system in section 4.3.3. 


\section{Chapter 5}

\section{Outlook and future work}

\subsection{Introduction}

Transmission-geometry X-ray diffraction as described here has proved to be remarkably successful. However, the full potential of this technique has yet to be realised. As described in Chapter 3, the data presented in this investigation has been obtained by, what is in essence, a very simple fixed apparatus. Scattering angles are defined by the fixed grooves of the collimator block and the beam geometry is such that, a rather coarse scattering voxel is created for sampling a material under investigation.

Given what has been achieved so far with the basic apparatus, the potential could be further un-locked through development of a new experimental apparatus, and possible use of alternative detector systems. Section 5.2 details improvements in the design that can be realised through the use of readily available commercial equipment.

In terms of future measurements an improved apparatus would allow 
further measurement of (for instance) the martensite phase transformation and expansion into areas currently not possible with the basic setup. These are detailed in section 5.3.

As detailed previously in section 4.4, a bench-top system such as this will always be fundamentally limited by the available X-ray flux, and as such, will not compete directly with the capabilities of a high energy diffraction beamline at a synchrotron such as the ESRF. However, such a system can prove complementary, providing for example, a coarse map (such as that produced for martensite) of a sample, indicating areas of interest that may be probed more finely with the higher precision attainable at a synchrotron. Furthermore it is important to recognise, there are a great deal of industrial applications where synchrotron sources are simply not viable given that in-situ measurements are required, sometimes in challenging environmental conditions not suitable for delicate detector systems. It is in these applications (such as that described in Appendix B) where the bench-top configurations are particularly suitable.

\subsection{Future equipment development}

Throughout the EDXRD measurements, a $1 \mathrm{~mm}$ by $10 \mathrm{~mm}$ beam geometry has been used, providing a reasonable angular resolution whilst maintaining a useful incident flux. For the HPGe detector, investigating $3 \mathrm{~mm}$ thick steel samples this yielded an elastic scatter flux of the order several hundred counts per second with the $225 \mathrm{kV}$ tube operating at $100 \mathrm{kV}$ and a beam current of $10 \mathrm{~mA}$. For typical measurements (for example on the $3 \mathrm{~mm}$ thick austenite- 
phase samples), sufficient statistics are acquired in around 30 minutes. One of the key problems encountered in later work (and one precluding the measurement of residual stress in samples) is the size of the sampling volume. Using the low-alloy ferritic steel as an example, the typical grain size is of the order $10 \mu \mathrm{m}$. With the scattering voxel defined, this results in approximately 30 million grains being sampled, any subtle effects such as residual stress would be lost in such a large distribution (for reference, a typical measurement at the ID15A beamline at the ESRF on the $25 \mathrm{~mm}$ thick samples using a 0.15 by $0.15 \mathrm{~mm}$ incident beam, samples 150,000 grains).

One of the key aims of an updated apparatus would therefore be further collimation of the incident and scattered beams to reduce the voxel size. In Fig. 5.1 this would be achieved using a pair of HUBER tungsten bladed crossslit screens for the incident beam, and a separate slit configuration (again HUBER cross-slit screens) to shape the scattered beam. Tightening the collimation in this way should also reduce the angular blurring (the range of possible scatter angles accepted by the detector), thus improving the resultant peak resolution. Further to improving collimation, a second area to look at is the peak energy of the incident beam. Photons of up to $100 \mathrm{keV}$ have been used in this investigation, at a fixed scattering angle of $9^{\circ}$, proving to be acceptable for $3 \mathrm{~mm}$ thick steel samples. However effects such as residual stress are difficult to re-produce in such thin samples $(25 \mathrm{~mm}$ thick samples were for example, used in the ESRF experiments). In order to broaden the capabilities of the system, higher X-ray energies, and thus smaller scattering angles must be used. Referring to the XCOM data, the mass attenuation coefficient $\mu / \rho$ at $100 \mathrm{keV}$ is $3.317 \times 10^{-1} \mathrm{~cm}^{2} / \mathrm{g}$, yielding for $3 \mathrm{~mm}$ steel the ratio 


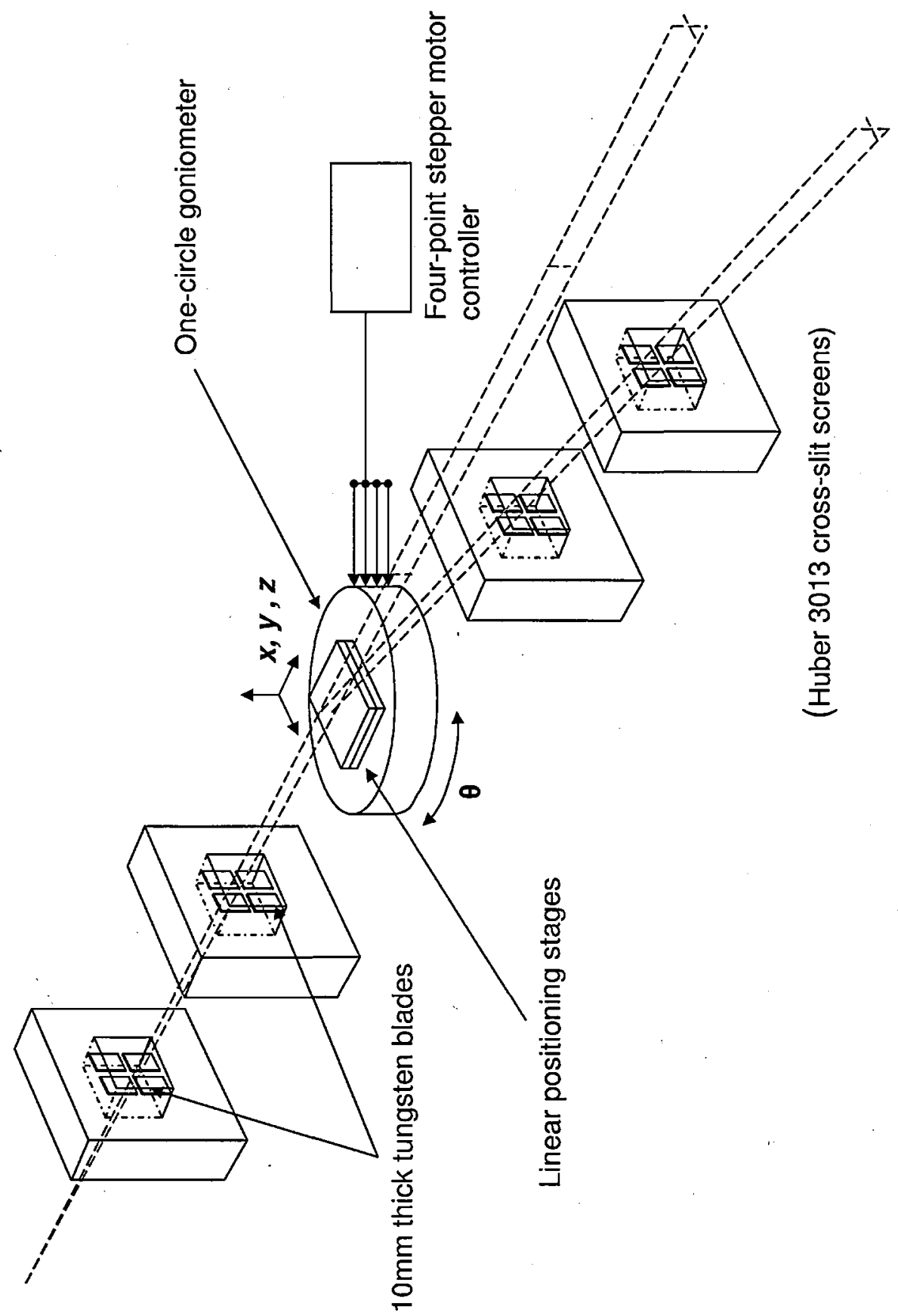

Figure 5.1: Updated EDXRD apparatus, including improved collimation (up to $200 \mathrm{keV} \mathrm{X}$-rays) and sample positioning in all three axis with rotation provided by a one-circle goniometer and linear stages. 
of transmitted to incident beam intensity, $I / I_{0}$, of 0.416 . Increase the thickness to $10 \mathrm{~mm}$ however, and $I / I_{0}$ drops to 0.054 . Given the weakness of the coherent scatter intensity compared to the transmitted intensity, this would, to all intents and purposes, render it impossible to utilise transmission XRD at this thickness. However, if the photon energy is increased to $200 \mathrm{keV}$ (for example utilising the $225 \mathrm{kV}$ X-ray tube at HMS Sultan's facility) the mass attenuation coefficient is $1.46 \times 10^{-1} \mathrm{~cm}^{2} / \mathrm{g}$, yielding for $3 \mathrm{~mm}$ steel an $I / I_{0}$ of 0.708 , and for $10 \mathrm{~mm} I / I_{0}$ of 0.317 , plausible for transmission XRD on a bench-top system. Such a configuration would require that the blades on the slit screens be made of $10 \mathrm{~mm}$ thick tungsten in order to ensure sufficient collimation of the higher energy beam.

In parallel to improved collimation/the use of high beam energies, improved sample positioning would prove useful for, for example, profiling with depth (refer to section 5.3). Again this could be quite easily achieved with standard $\mathrm{x}-\mathrm{y}$ stages, which combined with a $\mathrm{z}$-axis stage and one-circle goniometer would provide the full range of sample movements with respect to the incident beam.

Perhaps a more exciting prospect however, would be the use of $\dot{a}$ hybrid pixel detector such as Medipix [39], to collect multiple energy dispersive spectra, and thus, simultaneous profiling at different depths in the sample. Medipix is a spin-off from CERN, consisting of a CMOS (Complementary Metal Oxide Semiconductor) readout chip with 256 by $25655 \mu \mathrm{m}$ pixels, where each individual readout pixel is connected to an individual pixel on the sensor (i.e. detector) chip using an indium 'bump bond' [58]. The separation of readout chip and sensor chip allows for the utilisation of continuing 
developments in commercial silicon CMOS technologies while using materials more efficient at detecting higher energy X-rays, for example CdTe, for medical imaging purposes [59]. Furthermore the Medipix2 detector has been demonstrated running off a standard USB port from a laptop and is able to be connected to other medipix chips on three sides to form a detector array [60].

Another very recent development is that of the PILATUS 6M detector (DECTRIS Ltd, Switzerland) [61], first demonstrated on a synchrotron beamline (X06SA at the SLS) in November 2006, and in use from June 2007. This system is again a hybrid pixel device, consisting of silicon sensor chip bump-bonded to separate readout chip. The $6 \mathrm{M}$ detector consists of 5 by 12 modules with $2463 \times 2527$ pixels and a total active area of $424 \times 435 \mathrm{~mm}^{2}$ [62].

\subsection{Further investigation}

Development of the experimental apparatus to higher levels of precision opens up further avenues of measurement not possible with the current system. In the simplest case of precision sample positioning, the martensite mapping described in section 4.3.3 could be enhanced to include profiling at different depths as well as the $\mathrm{x}-\mathrm{y}$ positions of the map (refer to Fig. 5.2). Given that martensite platelets do not form uniformly throughout the bulk of the austenite grains, this could provide information on the depth to which the transformation is occurring under different regimes of residual stress/quenching in liquid nitrogen. 


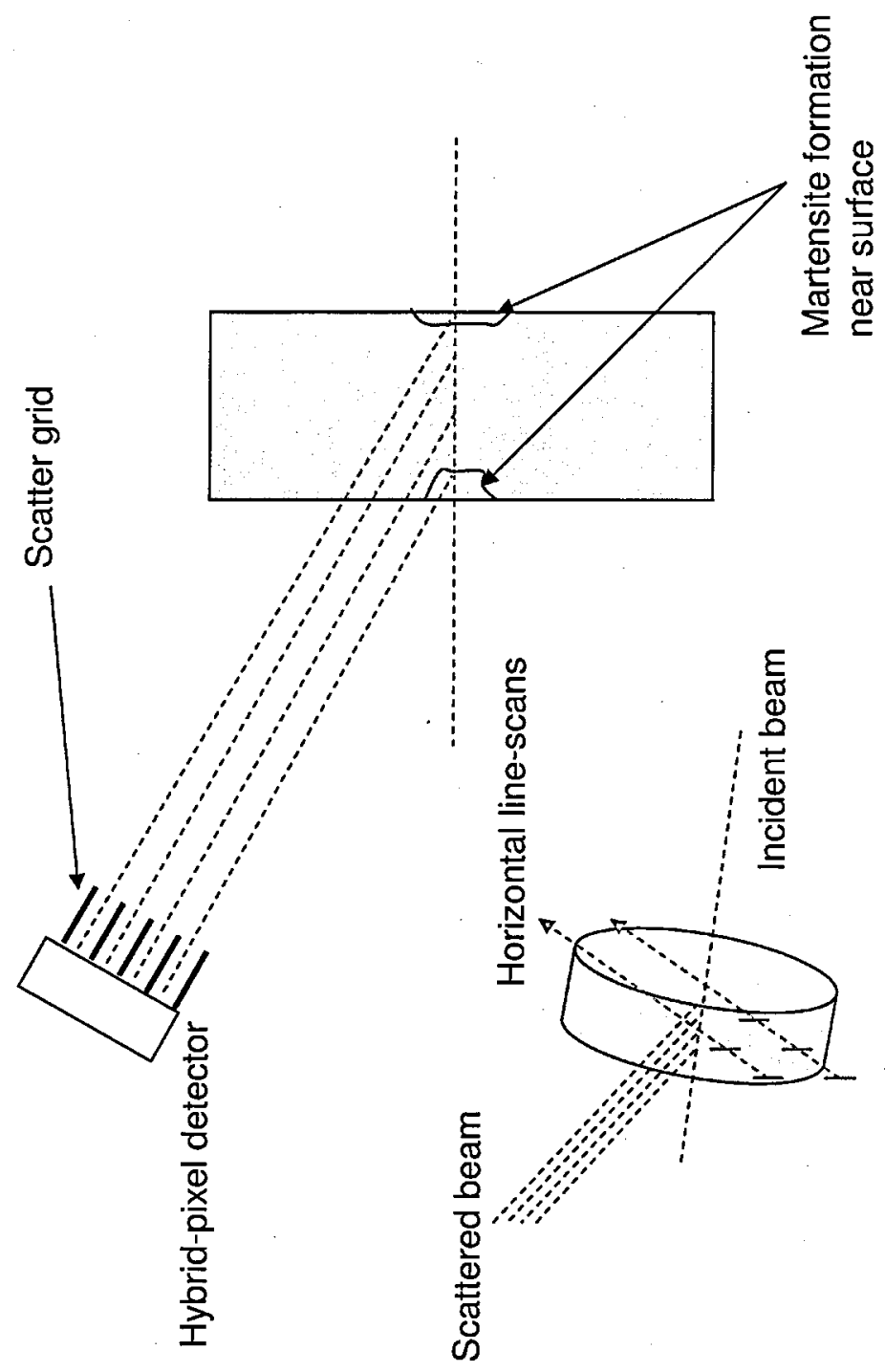

Figure 5.2: Updated Martensite mapping technique. Increased collimation of the incident beam will allow more precise mapping of the sample, and thus the areas where martensite is forming. At the same time however, a depth profile could be achieved through either moving the sample and taking individual measurements, or through simultaneous EDXRD measurements at different depths using a hybrid pixel detector as indicated. 
A further area opened up by improved beam collimation and the greater penetration offered by higher energies is that of mapping residual stress which is currently limited to high energy beamlines such as ID15A at the ESRF. Mapping of residual stress has been briefly attempted on the Surrey apparatus, but did not prove optimal due to insufficient resolution and the nature of the sample itself. As shown in section 4.3.4 the effect of larger applied stresses on lattice spacings is clearly visible with the current apparatus, though poor resolution of the individual lattice spacings manifested as asymmetric gaussians, results in the inability to accurately measure those changes. More refined beam geometries and a higher beam energy (minimising differential attenuation effects) have already enabled high precision measurements at the ESRF. Furthermore in order to generate residual stress states, the samples themselves must be thicker ( $25 \mathrm{~mm}$ thick samples were used at the ESRF). Increasing the viable sample thickness to $10 \mathrm{~mm}$ may therefore allow more subtle residual stresses to be observed in the same way that large applied stresses can be with the current system.

One of the persistent concerns in terms of damage to the 304L stainless steel, is corrosion brought about by high temperatures and stress. In the same way stress has been investigated dynamically in this project, a dynamic measurement of the complete stress corrosion cracking process may be plausible. A stainless steel sample identical to those used here could be fixed inside a 'collar' of boiling $\mathrm{MgCl}$ solution contained inside a glass envelope, accelerating the stress corrosion cracking process from years to days. Given that the attenuation of X-rays by the glass and solution should be minimal, it may be possible to perform transmission XRD measurements at differ- 
ent stages of the corrosion and crack propagation process inside the sample, yielding better understanding of this process.

There are potentially many more areas this system could be turned to, in terms of components and damage to steel. One possibility discussed, but never investigated is that of neutron damage, and possibly the combination of environmental effects such as neutron damage and SCC on the steel. Indeed, this need not be limited to work on steel samples; as shown briefly in section 4.3.1 even limited by the energies available on the lower power X-ray tubes, lattice spacing changes in bulk low-density samples, including novel nanopowders could be investigated. 


\section{Chapter 6}

\section{Conclusions}

X-ray diffraction is usually thought of as a surface analysis technique. Here, a broad account of X-ray diffraction in a transmission geometry for bulk analysis has been given.

Elastic scattering of $\mathrm{X}$-rays has been introduced with a classical derivation of the Thomson scatter cross section for a single electron. Modification of this relationship with the atomic form factor yields the coherent (Rayleigh) scattering cross section for many electron atoms. In the case of crystalline structures, Rayleigh scattered X-rays interfere constructively producing the distinct patterns associated with Bragg diffraction.

The techniques and experimental apparatus used for coherent scattering measurements have been introduced, including description of the construction and operation of X-ray tubes and the principles of semiconductor radiation detectors used in this investigation.

A comprehensive account has been given of work carried out using the energy dispersive configuration, where initial measurements on low density 
samples, including sample heating and associated changes in lattice spacing, have been used to configure the system geometry.

Energy dispersive X-ray diffraction measurements with both low-alloy ferritic, and 304L austenitic stainless steel, including plastically deformed (through tensile loading) 304L samples provided by HMS Sultan have followed. While the resultant spectra from these tensile-loaded samples showed no change in lattice spacing positions (the manifestation of slip deformation in the sample), they did however demonstrate unexpected and striking changes for the most highly deformed sample. These changes are likely due to a phase transformation from the face-centred cubic, to body-centred tetragonal structure of the martensite phase, which can occur in this particular alloy. This apparatus itself could be further developed for dynamic measurements of stress corrosion cracking in samples of interest to the NNPP.

Follow up work on the formation of martensite has been carried out on compressively loaded and quenched austenite-phase samples, designed to induce a residual stress field such that upon quenching in liquid nitrogen, martensite plates form inside the austenite grain boundaries. These measurements have been carried out in conjunction with a finite element model of the residual stress field in the sample, and demonstrate the same structural changes in the diffraction spectra in areas of high plastic strain, as observed previously in the most highly deformed tensile-loaded sample. These include the suppression of the peak corresponding to the $d_{220}$ lattice spacing, and a sharp increase in the intensity of the $d_{200}$ peak, supporting the assertion that martensite has been seen. This work further verifies the validity of the finite element code with regards to modelling residual stresses induced in the 
pipework specimens (of interest to the NNPP), in the context of cryogenic treatment to 'harden' steels against the effects of stress corrosion cracking.

Dynamic measurements involving tensile-loading in-situ have demonstrated the capability to detect the subtle lattice spacing changes produced while elastically deforming a $304 \mathrm{~L}$ austenite-phase sample. Changes in the $d_{311}$ spacing of $0.75 \%$ are detectable, and as demonstrated, refinements to the geometry can further improve the resolution of the $d_{311}$ peak, potentially allowing for smaller changes to be detected.

The use of angular dispersive X-ray diffraction in a parallel development to that of the energy dispersive technique has been briefly introduced, and is continued with an article in Appendix B.

The publications listed below and detailed in the Appendices include the aforementioned article, regarding the use of transmission X-ray diffraction in the angular dispersive configuration, for measurements of the phase transformation in steel, and published in Nuclear Instruments and Methods B [36]. An internal publication with regards to HMS Sultan's X-ray facilities [35] has been included in Appendix A, while a more recent publication in $\mathrm{Nu}$ clear Instruments and Methods A [50] as part of the proceedings of the 10th International Symposium on Radiation Physics is included in Appendix C.

- D.J. Garrity, P.M. Jenneson, S.M. Vincent and A.C. Williams. Deter- mination of X-ray Tube stability/suitability for use in X-ray transmission diffraction research Unpublished MoD report (2005)

- D.J. Garrity, P.M. Jenneson, R.D. Luggar and S.M. Vincent. Measurement of the phase transformation in sheet steel from the ferrite to. 
austenite phase using angular dispersive X-ray diffraction in a transmission geometry. Nuclear Instruments and Methods in Physics Research B 251 (2006) 197-200

- D.J. Garrity, P.M. Jenneson and S.M. Vincent. Transmission geometry X-ray diffraction for materials research. Nuclear Instruments and Methods in Physics Research A 580 (2007) 412-415 


\section{Appendix A}

\section{$225 \mathrm{kV}$ X-ray Tube}

The following internal HMS Sultan publication describes tests run on HMS

Sultan's $225 \mathrm{kV}$ X-ray tube in order to determine its status/operability. 


\title{
Determination of X-ray Tube Stability/Suitability for use in X-ray Transmission Diffraction Research
}

\author{
D.J. Garrity ${ }^{1}$, P. M. Jenneson ${ }^{1}$, S. M. Vincent ${ }^{2}$ and A. C. Williams ${ }^{2}$ \\ ${ }^{1}$ Department of Physics, School of Electronics and Physical Sciences, University of Surrey, Guildford, Surrey, \\ GU2 $7 X H, U K$. \\ ${ }^{2}$ Nuclear Department, Defence College of Electro-Mechanical Engineering, HMS Sultan, Military Road, Gosport, \\ Hampshire, POI2 3BY, UK
}

\begin{abstract}
The X-ray tube located in the former armoury building is the central apparatus in a collaborative effort with the University of Surrey into X-ray Transmission Diffraction for materials research.

Dosimeter measurements to compare dose rate to manufacturer specifications and the collection of energy spectra with a high resolution HPGe detector at nominal tube voltages $(100 \mathrm{kV}, 160 \mathrm{kV}, 225 \mathrm{kV})$; reveal an X-ray tube that is in very good working order, with ideal characteristics for use in the forthcoming X-ray Transmission Diffraction work, namely:

A smooth bremsstrahlung continuum generated from electron deflection in the tungsten anode, providing an ideal polychromatic source for energy dispersive measurements (and without the shift in the bremsstrahlung endpoint that is characteristic of a 'gassy' tube).

The production of sharp, characteristic tungsten lines that, with suitable filtration; may be used as a suitable quasimonochromatic source for angular dispersive measurements.
\end{abstract}

\section{Introduction}

In collaboration with the University of Surrey, the Nuclear Department at HMS Sultan is to investigate the potential of Transmission X-ray Diffraction for materials research within the Naval Nuclear Propulsion Programme.

The use of X-rays for imaging can be dated back to their first discovery over a century ago. In the intervening period, the field of X-ray imaging has developed along two parallel methods; that of X-ray diffraction/X-ray crystallography from the pioneering work of Laue and later Bragg, and that of radiography illustrated by Roentgen's famous radiograph of his wife's hand.

In more recent years there has been a great deal of focus on exploiting coherent (Rayleigh) scattered $\mathrm{X}$-rays for imaging purposes. Although the classical relationship for elastic scattering (an electromagnetic wave causes an electron to oscillate sympathetically producing a secondary wavelet of the same frequency) was discovered in the early $20^{\text {th }}$ century by Thomson and others, its potential in a transmission geometry has only come to light within the last twenty years.

The potential for coherent scattering was first realised following investigations into 'streaking' effects in transmission CT images. Imaging of water phantoms revealed this phenomenon to be the result of constructive interference between elastically scattered X-rays from neighbouring water molecules. Given the requirement for the scattered waves to be in phase, this phenomenon is strongly peaked in the forward (i.e. low angle) direction, at higher angles destructive interference increases. For crystalline structures with regular periodicity this scattering will manifest itself as Bragg diffraction. Since this discovery the technique, sometimes referred to as Low angle X-ray Scattering (LAXS), has been exploited in a wide range of fields from aviation security [1] through to industrial process control.

\section{Coherent Scattering and X-ray tubes}

The strength of the coherent scatter signal is a variable of atomic number $Z$ and the parameter ' $q$ ' the momentum transfer. The function of these two variables is the 'atomic form factor' $F(q, z)$ [2] which relates Rayleigh scattering for multiple, bound electrons to the classical relationship derived by Thomson for a single, unbound electron.

The momentum transfer is given by:

$$
q=2 \hbar k \sin \left(\frac{\theta}{2}\right)
$$

Thus ' $q$ ' and therefore by extension the scattering amplitude, is dependent on the energy of the incoming photon and the scattering angle This allows for two distinct forms of coherent scatter measurements; 1) angular dispersive, where energy is fixed and the scattering angle $\theta / 2$ varied, and 2) energy dispersive, whereby a fixed scatter angle for various energies is used. With both techniques the momentum-space distribution of the differential scatter cross section is being sampled, both require a high photon flux for reasonable measurement times, 2) requires a polychromatic source in conjunction with an energy resolving detector, 1) requires a filtered source and either a moving source or detector to sample different regions of momentum space. In both cases the requirements are fulfilled by a conventional industrial X-ray tube.

$\mathrm{X}$-ray tubes produce a broad, continuous bremsstrahlung spectrum, ideal for conducting energy dispersive measurements (particularly so 
with a tungsten anode). Higher intensities can only be achieved by using an expensive (a complete facility required) synchrotron source. The endpoint (i.e. maximum photon energy) of the continuous spectrum is determined by the tube potential $\mathrm{kV}_{\mathrm{p}}$. Maximum filament current/tube potential is limited by the effectiveness of the cooling system that removes heat from the anode.

In addition to the bremsstrahlung continuum, characteristic X-ray peaks are generated from removal of inner $k$-shell electrons and subsequent de-excitation of L-shell ( $k_{\alpha}$ X-rays) or M-shell $\left(k_{\beta}\right.$ $X$-rays) electrons in the target anode. These characteristic X-ray lines are sharp (typically $<0.001 \AA$ ) and of high intensity, which when combined with a suitable filter allows the source to be quasi-monochromated for use in angular dispersive measurements.

\section{Status of the Current X-ray tube}

The tube located in the former armoury building comprises a tungsten target anode, with maximum power dissipation of $3000 \mathrm{~W}$. At maximum $\mathrm{kV}_{\mathrm{p}}$ $(225 \mathrm{kV})$ this corresponds to a filament current of $13 \mathrm{~mA}$.

The prime concern is the stability of the vacuum of the interior volume of the tube; this is evacuated upon manufacture to prevent interaction between electron beam and atmospheric gases. Should a tube become 'gassy' the electrons will impart some of their energy into ionising the interior gas, resulting in reduced maximum $X$-ray energy. In addition the generation of positive ions within the tube under high voltage conditions has the potential to lead to internal arcing and permanent damage.

To test the stability of the vacuum/overall effectiveness of the tube, spectra at standard operating $\mathrm{kV}_{\mathrm{p}}(225 \mathrm{kV}, 160 \mathrm{kV}, 100 \mathrm{kV})$ are taken in addition to measurement of Dose rate at $1 \mathrm{~m}$ from the source for comparison with quoted manufacturers values for a similar X-ray tube.

\section{Stability of vacuum/spectrum quality}

All spectra were collected with HPGe detector situated directly in the path of the primary beam. Given the intensity the X-ray tube is capable of generating, and the single photon counting nature of the detector, the filament current was reduced down to $0.3 \mathrm{~mA}$ and the detector positioned $8 \mathrm{~m}$ from the tube window. Further to this a pin-hole lead collimator was positioned in front of the detector to prevent pulse pile-up and saturation of the detector. Fig. 1 contains the spectrum obtained operating at $225 \mathrm{kV}, 0.3 \mathrm{~mA}$. The end-point energy of the spectrum occurs at $225 \mathrm{keV}$, indicating that the tube is not gassy. The strongest peak as expected is the tungsten $k_{\alpha}$ doublet; $k_{\alpha 1}$ visible at $59.5 \mathrm{keV}, k_{\alpha 2}$ at 58 $\mathrm{keV}$, the result of $\mathrm{k}$-shell electron ionisation and subsequent L-shell electron de-excitation (generation of $k_{\alpha l} / k_{\alpha 2}$ dependent on which L subshell the electron de-excites from). Tungsten $k_{\beta}$, the result of M-shell de-excitation, is visible at $67 \mathrm{keV}$. Given the quantity of lead used in the collimation of the primary beam before detection, and noting that the maximum photon energy of the primary beam exceeds the K-absorption edge for lead, characteristic lead $k_{\alpha}, k_{\beta} \mathrm{X}$-rays are visible $(75 \mathrm{keV}$ and $85 \mathrm{keV}$ respectively) in this spectrum.

The weak, unidentified peaks at energies, $24.5 \mathrm{keV}, 69.5 \mathrm{keV}, 73 \mathrm{keV}$ and $87.7 \mathrm{keV}$ are most likely the result of characteristic X-ray emission from the materials in a small detector window located directly in front of the $\mathrm{X}$-ray tube window (Fig. 3). Unfortunately information on the exact composition is not available; the detector consists of isobutene with a Mylar (Polyethylene Terepthalate) exterior, probably with a metallised coating.

Reducing $\mathrm{kV}_{\mathrm{p}}$ to $160 \mathrm{kV}$ generates the spectrum of figure 2. Once again the end-point energy is correct, the 'tail' of the bremsstrahlung continuum reaching $160 \mathrm{keV}$, confirming the stability of the vacuum. All expected characteristic peaks are present, the tungsten $k_{\alpha}, k_{\beta} \mathrm{X}$-rays are still the most prominent. With the reduction in $\mathrm{kV}_{\mathrm{p}}$ and thus the reduction in the amount by which maximum photon energy exceeds the $\mathrm{K}$-absorption edge for lead, a decrease in the relative intensity of the associated $k_{\alpha}, k_{\beta} \mathrm{X}$-rays is observed.

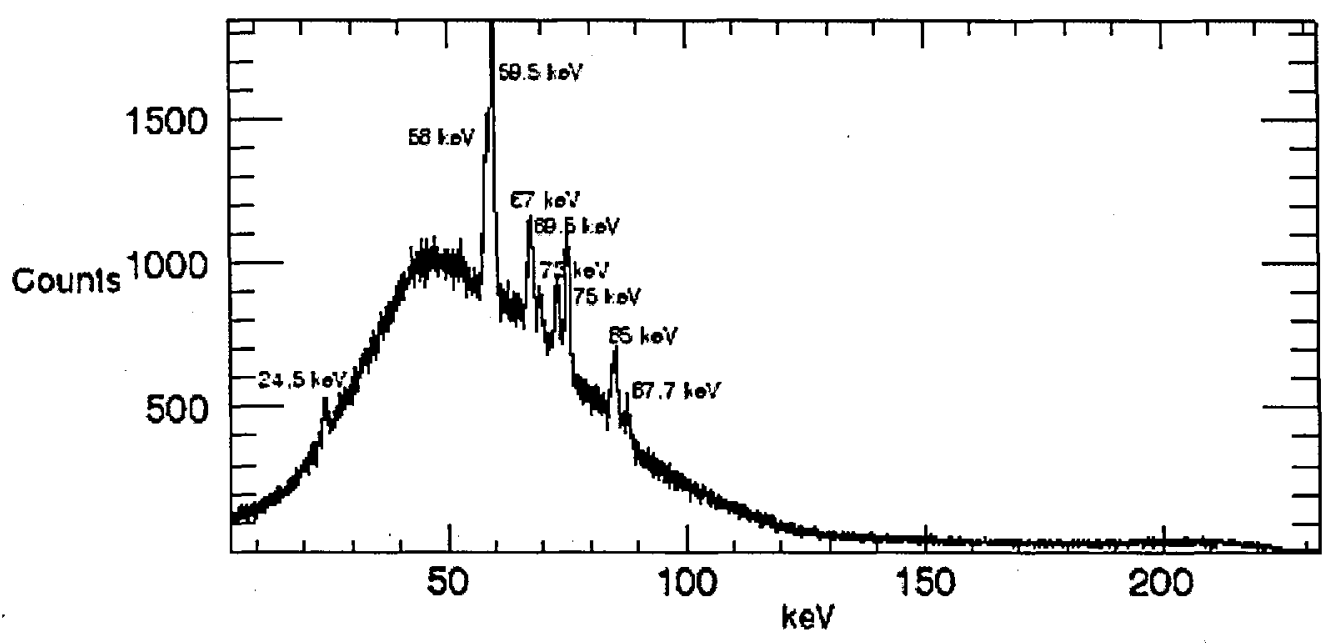

Fig. 1. Spectrum generated at $225 \mathrm{kV}, 0.3 \mathrm{~mA}$. Endpoint energy of $225 \mathrm{keV}$ appears to indicate that tube is not gassy. Characteristic tungsten lines; the $k_{\alpha}$ doublet and $k_{\beta}$ are visible at the correct energies. 


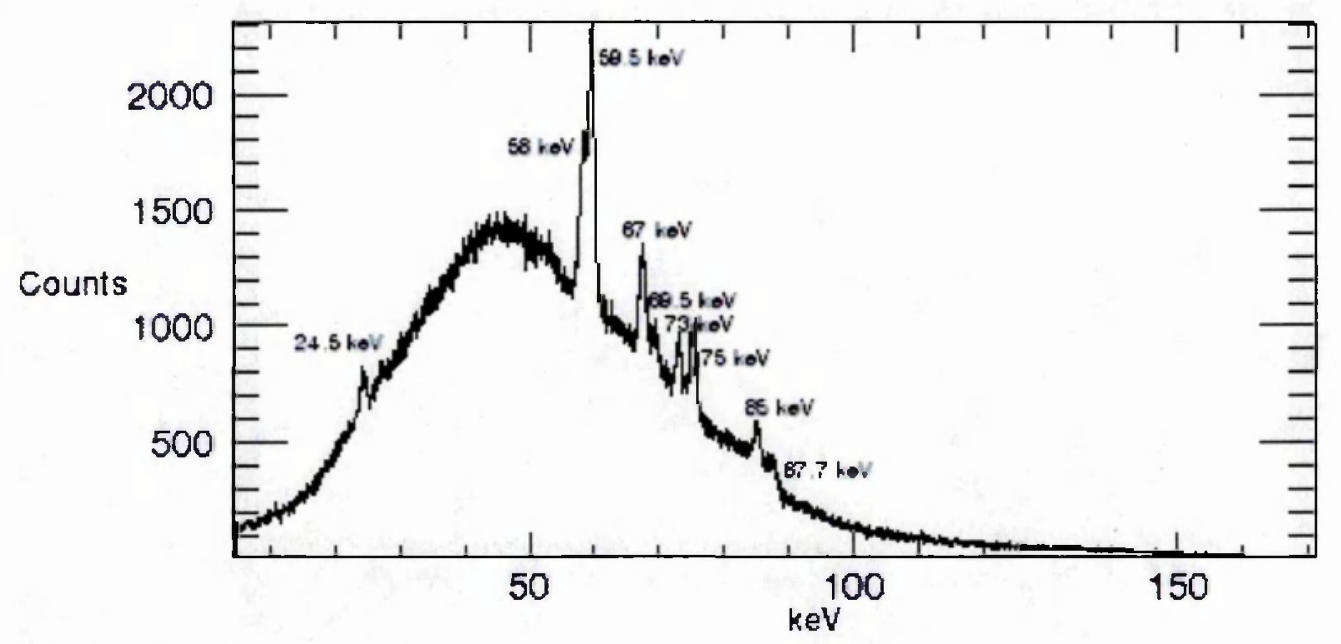

Fig. 2. $\mathrm{kV}_{\mathrm{p}}$ reduced to $160 \mathrm{kV}$. Bremsstrahlung endpoint energy is clearly visible at $160 \mathrm{keV}$, confirming that the tube is not 'gassy'. Characteristic peaks of Tungsten and lead are still present, the latter in particular at reduced intensity given the reduction in the number of photons exceeding the lead $\mathrm{K}$-edge.

The final spectrum collected at a tube potential of $100 \mathrm{kV}$ is shown in Fig. 4. The stability and quality of the spectrum in this region are of particular interest given that $100-160 \mathrm{kV}$ are amongst the most commonly used $\mathrm{kV}_{\mathrm{p}}$ for coherent scatter measurements. The end-point energy is clearly not less than $100 \mathrm{keV}$ though it slightly less well defined than the previous spectra, possibly the result of minor fluctuations in $\mathrm{kV}_{\mathrm{p}}$. Given the smaller energy scale the tungsten $k_{\alpha}$ doublet is easily distinguishable as such. The characteristic lines for lead are very weak now that the gap between Kedge and maximum photon energy has narrowed. Tungsten $k_{\alpha} / k_{\beta}$ are also of a much reduced relative intensity compared to the peak of the bremsstrahlung continuum.
In addition to the collection of spectra at the three different $\mathrm{kV}_{\mathrm{p}}$ of interest, the dose rate at $1 \mathrm{~m}$ was taken at various combinations of $\mathrm{kV}_{\mathrm{p}}$ /filament current to compare to a newly manufactured X-ray tube. The dose (equivalent dose) and corresponding manufactures quotes are shown in Table 1. Dose was measured on a NE Technology 2571 Ionisation chamber inside a water phantom, positioned directly in the path of the primary beam. The quoted manufactures specifications are for a GE Inspection Technologies 'ISOVOLT $225 \mathrm{M} 2 / 0.4-3.0$ ' X-ray tube [3], capable of up to $225 \mathrm{kV}$ at $13 \mathrm{~mA}$. The existing tube compares very favourably, exceeding the quoted values by a factor of approximately two.

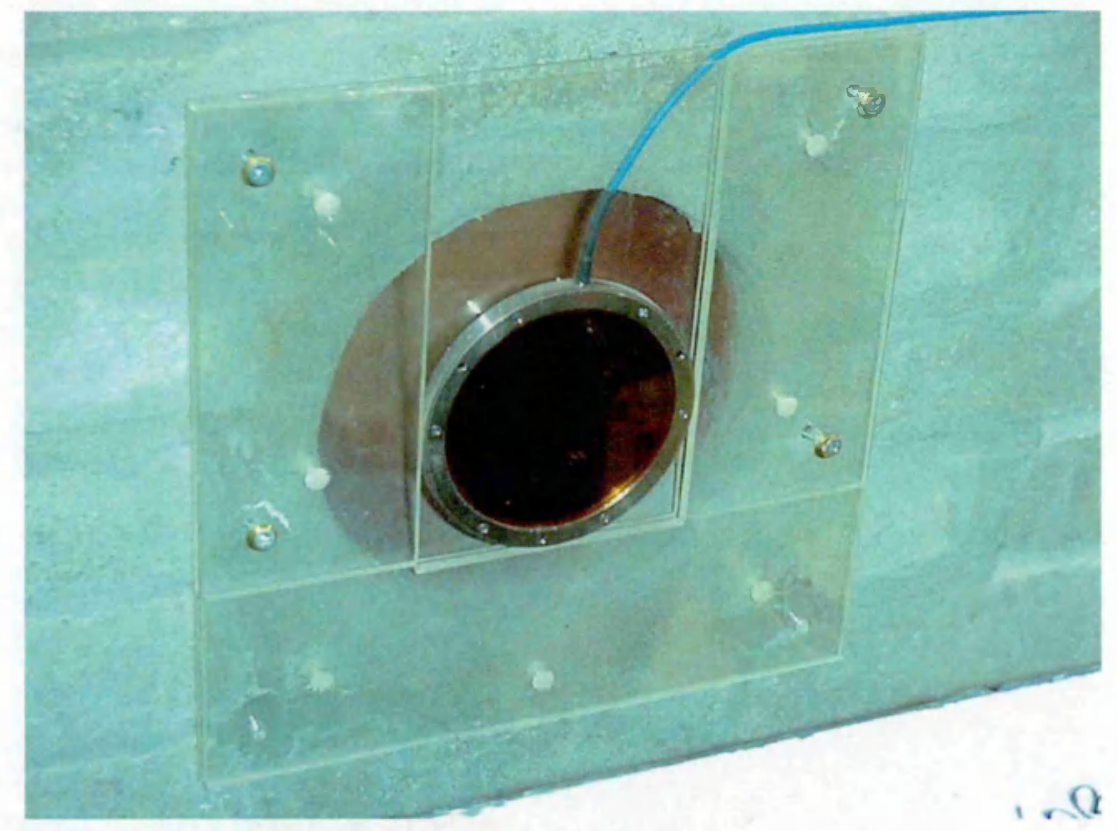

Fig. 3. Detector situated in front of the X-ray tube window, affixed to the beam port in the concrete 'shield wall'. The weaker peaks observed in the spectra gencrated are most likely due to X-ray fluorescence of the various constituent materials, specific information on its construction is unavailable. 


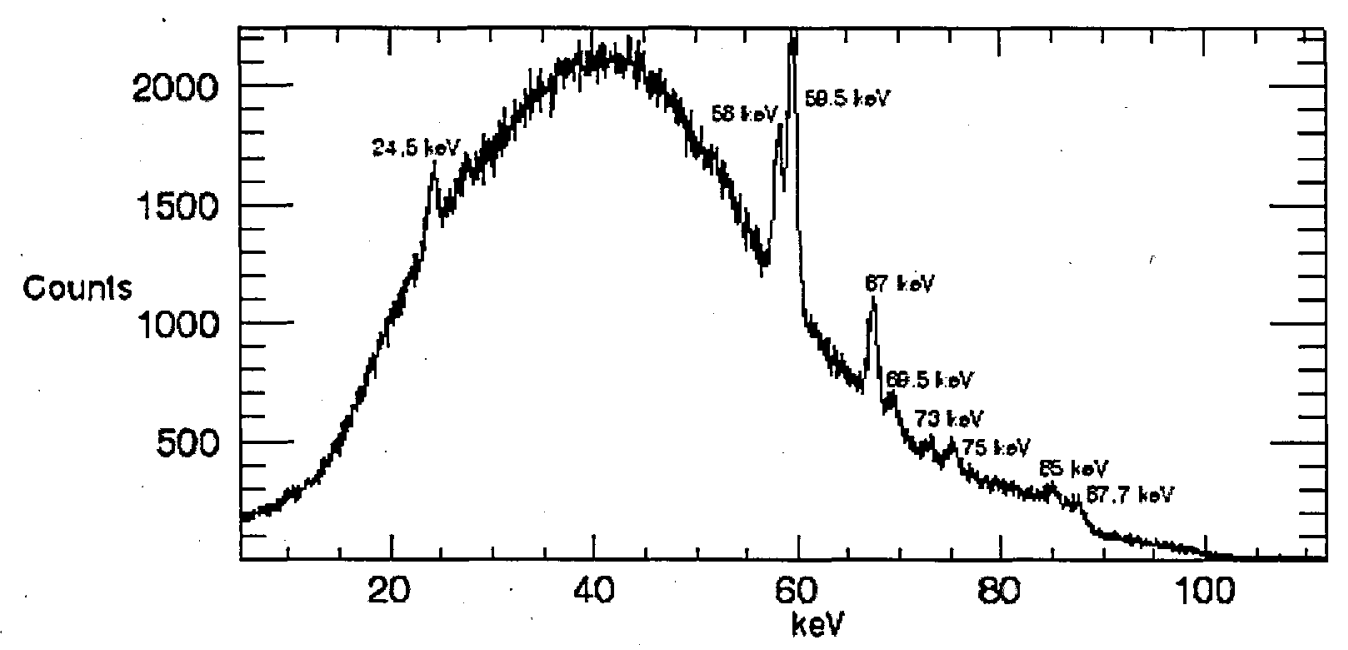

Fig. 4. Final spectrum, collected at $100 \mathrm{kV}$. Lead $k_{a}, k_{\beta}$ peaks are now very weak, there is also a significant reduction in relative intensity of tungsten characteristic peaks.

Table 1. Values for the dose-rate of the existing tube against manufacturer specified dose-rate.

\begin{tabular}{cccccc}
\hline \multicolumn{3}{c}{ Existing Tube } & \multicolumn{3}{c}{ Manufactures Quotes } \\
$\mathrm{KV}_{\mathrm{p}}$ & $\mathrm{mA}$ & $\mathrm{Sv} / \mathrm{hr}$ & $\mathrm{KV}_{\mathrm{p}}$ & $\mathrm{mA}$ & $\mathrm{Sv} / \mathrm{hr}$ \\
\hline 225 & 13 & 24.0 & 225 & 13 & 12.93 \\
225 & 7 & 12.92 & 225 & 7 & 6.92 \\
225 & 1.4 & 2.32 & 225 & 1.4 & 1.24 \\
160 & 10 & 10.67 & 160 & 10 & 4.64 \\
160 & 4 & 4.23 & 160 & 4 & 1.62 \\
160 & 2 & 2.07 & 160 & 2 & 0.91 \\
\hline
\end{tabular}

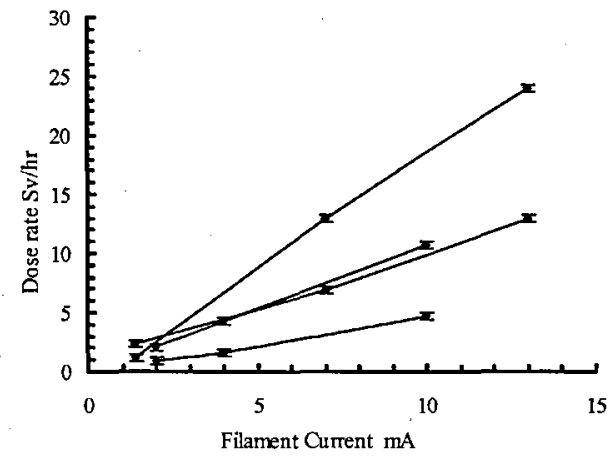

Fig. 5. Linearity of Dose rate with different tube voltages, from top to bottom: (measured); $225 \mathrm{kV}, 160 \mathrm{kV}$, (quoted); $225 \mathrm{kV}, 160 \mathrm{kV}$.

\section{Conclusion}

The X-ray tube at HMS Sultan is the central apparatus in a collaborative effort with the University of Surrey into X-ray Transmission Diffraction for materials research. Dosimeter measurements and collection of energy spectra with a HPGe detector at various tube voltages reveal an $\mathrm{X}$-ray tube that is in very good working order, with ideal characteristics for use in X-ray Transmission Diffraction work.

The smooth bremsstrahlung continuum generated from electron deflection in the tungsten anode provides an ideal polychromatic source for energy dispersive measurements; no shift in the bremsstrahlung endpoint was observed, which would have indicated a 'gassy' tube. In addition to this, sharp tungsten characteristic lines are produced, with suitable filtration the $k_{\alpha}$ lines in particular may be used as a suitable quasimonochromatic source for angular dispersive measurements.

\section{References}

[1] R. D. Luggar, J. A. Horrocks, R. D. Speller, R.J. Lacey, "Low angle X-ray Scatter for explosives detection: a Geometry Optimization" Appl. Radiat. Isot. Vol. 48. No.2 pp. 215-224, 1997

[2] J. H. Hubbell, W. J. Veigele, E. A. Brigg, R. T. Brown, D. T. Cromer R. J. Howerton, "Atomic Form factors, incoherent scattering functions and photon scattering cross sections" J.Phys. Chem. Ref. Data 4(3) (1975), 471.

[3] GE Inspection Technologies. http://www.geinspectiontechnologies.com/products/ XRay/Tubes/Stationary/xray_tubes.html 


\section{Appendix B}

\section{Phase transformation in steel}

The following is a Nucl. Instr. and Meth B publication, regarding the measurement of the phase transformation in steel from the ferrite to austenite phase using transmission $\mathrm{X}$-ray diffraction. 


\title{
Measurement of the phase transformation in sheet steel from the ferrite to austenite phase using angular dispersive X-ray diffraction in a transmission geometry
}

\author{
D.J. Garrity ${ }^{\text {a }}$, P.M. Jenneson ${ }^{\mathrm{a}, *}$, R.D. Luggar ${ }^{\mathrm{a}}$, S.M. Vincent ${ }^{\mathrm{b}}$ \\ ${ }^{a}$ Department of Physics, School of Electronics and Physical Sciences, University of Surrey, Stag Hill, Guildford, Surrey GU2 $7 X H$, UK \\ b Nuclear Department, Defence College of Electro-Mechanical Engineering, HMS Sultan, Military Road, Gosport, Hampshire PO12 3BY, UK
}

Received 11 January 2006; received in revised form 2 May 2006

Available online 27 June 2006

\begin{abstract}
An angular dispersive X-ray diffraction arrangement, utilising an image intensifier and X-ray filtering, has been developed to measure the ferrite to austenite phase transformation in an online steel foundry process line. Given the degradation in resolution associated with transmission measurements, the extreme phases have been used to calibrate the system. The most significant differences between these two phases are found to occur at scattering angles of between $6^{\circ}$ and $8^{\circ}$. The results obtained from measurement of the radially integrated profile in this region whilst heating a ferrite-phase sample towards the phase transformation temperature are consistent with the expected trend, demonstrating the requirement for uniform temperature between the ferrite and austenite phase samples in order to observe the phase transformation.
\end{abstract}

(C) 2006 Elsevier B.V. All rights reserved.

PACS: $61.10 . \mathrm{Nz} ; 61.82 . \mathrm{Bg}$

Keywords: Transmission X-ray diffraction; Steel phases

\section{Introduction}

$\mathrm{X}$-ray diffraction can be used in a transmission geometry to study the nature of bulk materials and has already been developed as an inspection technique in fields such as medicine, industrial process control and aviation security [1]. In the work reported here, angular dispersive X-ray diffraction (ADXD) is being developed to measure the phase transformations in hot rolled steel from the ferrite to the austenite phase.

Whilst X-ray diffraction is often considered as a surface analysis technique whereby $\mathrm{X}$-rays are coherently (Rayleigh) scattered from the top few layers of atoms, it is equally suitable to bulk analysis provided the X-rays have

\footnotetext{
* Corresponding author. Tel.: 4441483 689419; fax: +44 1483686781.

E-mail address: p.jenneson@surrey.ac.uk (P.M. Jenneson).
}

sufficient energy to overcome the attenuation within the material. The diffraction data yields information relating to the crystalline structure of the material and therefore provides a non-invasive probe for measuring, for example, the phase transformation.

The use of energy dispersive X-ray diffraction (EDXD) provides identical data to ADXD but employs delicate high energy resolution devices such as hyper-pure germanium (HPGe) detectors. ADXD has the advantage that the equipment required (a position sensitive detector and the use of X-ray beam filtration), is more suited to the extreme environmental conditions found next to a steel foundry hot roller process line.

\section{Theory}

Coherent scattering of electromagnetic waves from a single unbound electron is described classically by the 
Thomson scattering cross section. For multiple, atomic electrons, the modified (Rayleigh) scattering cross section is based upon the form factor approximation [2] where:

$\frac{\mathrm{d} \sigma_{R}}{\mathrm{~d} \Omega}=\frac{r_{0}^{2}}{2}\left(1+\cos ^{2} \theta\right)[F(q, Z)]^{2}$.

The atomic form factor $F(q, Z)$ relates Rayleigh scattering for multiple, bound electrons, to the classical relationship derived by Thomson for a single unbound electron, with the corresponding momentum transfer, $q$, given by:

$q=2 \hbar k \sin (\theta / 2)$.

Thus $q$ and by extension, the scattering amplitude, is dependent on the energy of the incoming photon and the scattering angle. Angular dispersive measurements utilise a fixed-wavelength source with a two-dimensional position-sensitive detector to investigate all regions of low momentum transfer (low scattering angles), producing a characteristic signature of the material under investigation.

For amorphous materials, such as water, the effect is subtle; scattering from water molecules produces broadly featureless spectra [3]. With an ordered periodic array such as steel, scattering gives rise to a series of sharp peaks as associated with Bragg diffraction. Rather than calibrating the resultant spectra in terms of energy it is more useful to calibrate in terms of the parameter $x$ which is usually given in units of $\AA^{-1}$ :

$x=\frac{1}{\lambda} \sin (\theta / 2)$.

Thus having performed the calibration in terms of $x$, the lattice spacing $d$ may be obtained simply from $x=1 / 2 d$. In angular dispersive measurements scattering over all angles simultaneously produces a characteristic diffraction image corresponding to regions of constructive/destructive interference. In order to obtain the characteristic signature, a radial integration using the centre of the diffraction image pattern as the origin generates a profile in terms of $\theta$ versus intensity, which may then plotted in terms of $x$ as described above.

\section{Experimental apparatus}

All X-ray measurements were carried out in a dedicated lead-shielded X-ray room with the equipment remotely operated from an adjacent control room. The experimental arrangement used for transmission angular dispersive diffraction studies utilised a Thomson TH9428HP X-ray image intensifier and is shown schematically in Fig. 1.

The X-ray source used was a $225 \mathrm{kVp}$ tungsten reflection target X-ray tube (Comet MXR225/22 powered by a Gulmay CP-225 supply) mounted inside a $10 \mathrm{~mm}$ leadlined $(\mathrm{Pb})$ enclosure in order to remove stray scatter produced from the X-ray tube, therefore ensuring that the resultant measured scattering was produced only from the sample being studied. The X-ray tube was operated at a potential of $100 \mathrm{kVp}$ and $30 \mathrm{~mA}$ throughout, with inherent filtration provided by an output window consisting of a $20 \mathrm{~mm}$ diameter, $1 \mathrm{~mm}$ thick disk of beryllium.

The X-rays exiting the front window of the X-ray tube were further collimated by a $20 \mathrm{~cm}$ long copper-coated lead collimator into a $1 \mathrm{~mm}$ diameter pencil beam. For optimal measurement times the centre of the collimator was positioned over the region of most intense X-ray flux using micro-positioners, while the flux was recorded using a NE Technology Limited Farmer dosimeter (model 2670) with a $0.6 \mathrm{~cm}^{3}$ ionisation chamber (type 2571). An X-ray filter consisting of $250 \mu \mathrm{m}$ of hafnium was then placed over the entrance hole of the collimator; this removed any scattering produced by the filter itself. The filter was chosen based on the K-edge filtering technique described in our previous work [4], with the $59.3 \mathrm{keV}$ characteristic X-ray of tungsten quasi-monochromated as demonstrated in Fig. 2. This had the effect of increasing the definition of the diffraction rings shown in Fig. 3, while only reducing the intensity of the characteristic lines by $30 \%$.

It can clearly be seen that the unfiltered diffraction rings are broader than those for the filtered case. Apart from the removal of the filter, the system parameters remained unchanged during both of these acquisitions.

The steel sample was positioned $10 \mathrm{~mm}$ from the front of the collimator and held in place with a plastic clamp.

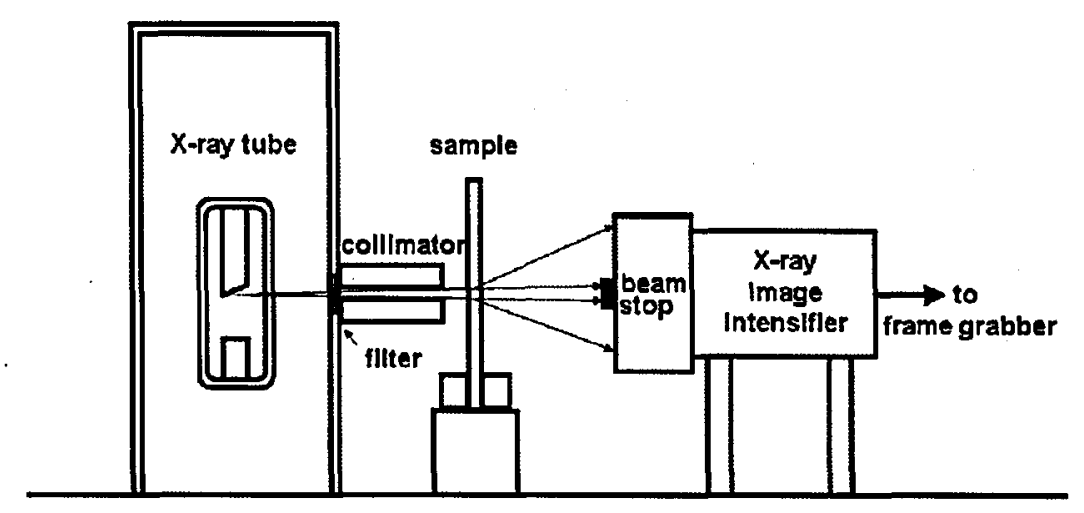

Fig. 1. The apparatus used for transmission angular dispersive diffraction measurements. 


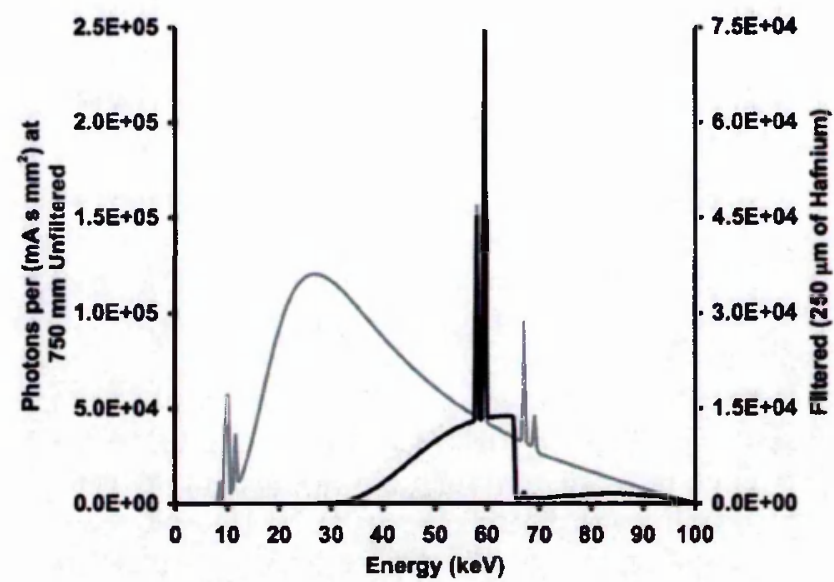

Fig. 2. The $X$-ray spectra simulated using the IPEM Report 77 Spectrum Processor software for a tungsten target tube with an angle of $22^{\circ}$ run at $100 \mathrm{kVp}$. Unfiltered and filtered spectra using $250 \mu \mathrm{m}$ of hafnium are shown.

It was placed on an elevating table in order to ensure that the centre of the sample was irradiated.

The front screen of an X-ray image intensifier (XRII) was positioned $30 \mathrm{~cm}$ from the sample to give an angular field of view of up to $20^{\circ}$. The XRII used was a Thomson TH9428HP tube optically coupled to a peltier-cooled Pulnix TM-80 high resolution integrating CCIR format CCD camera, the XRII had a $1 \mathrm{~mm}$ aluminium cover over the entrance window. A beam stop consisting of a $20 \mathrm{~mm}$ diameter, $8 \mathrm{~mm}$ thick disc of lead was placed in the centre of the aluminium cover to cut out the primary X-ray beam. The CCD integration time (1-3000 ms) and video output signal were managed by a Matrox Pulsar 10-bit framegrabber card installed in a remote $\mathrm{PC}$, allowing for control and processing of the video signal in to either projection images or radially integrated intensity versus angle plots.

During the heating of the steel samples a Fluke digital thermocouple thermometer model 54 II was used with a K-type thermocouple to monitor the sample temperature. The sample itself was held in a Macor ceramic clamp to prevent heat loss, while the thermocouple was spot-welded to the sample to ensure a good contact. Heating of the sample up to $800^{\circ} \mathrm{C}$ was then carried out using a high power butane/propane gas torch.

\section{Results and discussion}

Ferrite (mild steel) and austenite (stainless steel) samples were analysed using the transmission ADXD apparatus in order to determine where the most significant changes between the two phases occur. As ferrite steel is annealed by raising its temperature, its lattice spacings transform into that of the austenite phase. Therefore as demonstrated in Fig. 4, a comparison of the diffraction patterns of ferrite and austenite phases enables the identification of the region where the two phases differ the most significantly.

One of the most significant regions is that between $6^{\circ}$ and $8^{\circ}$, relating to the $d_{002}$ and $d_{110}$ lattice spacings in the ferrite phase (JCPDS 33-474) [5] and the $d_{200}$ and $d_{111}$ lattice spacings in austenite phase (JCPDS 33-397) [5]. The poor energy and angular resolution inherent with using transmission geometries imply that, rather than clearly

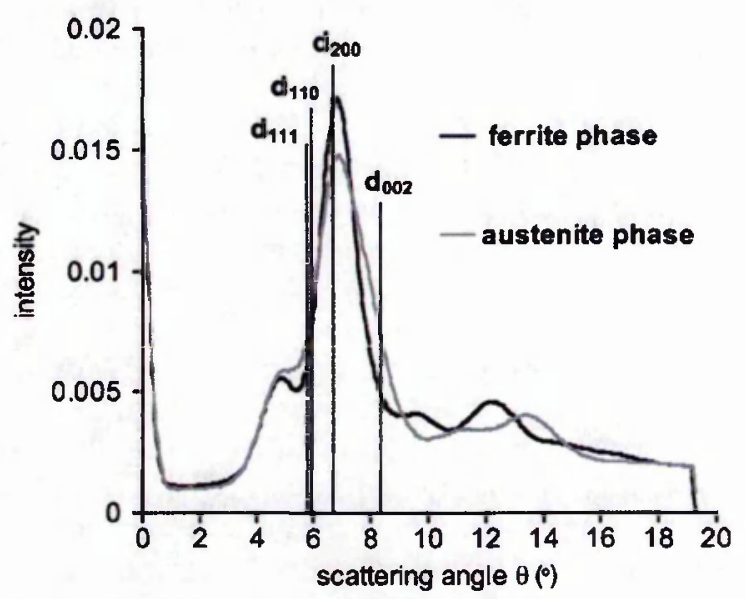

Fig. 4. Scattering angle of the $59.3 \mathrm{keV} X$-rays for $2 \mathrm{~mm}$ samples of ferrite and austenite phase steel, including the positions [5] of the $d_{110}, d_{002}$ lattice spacings of ferrite and $d_{111}, d_{200}$ lattice spacings of austenite phase steel.
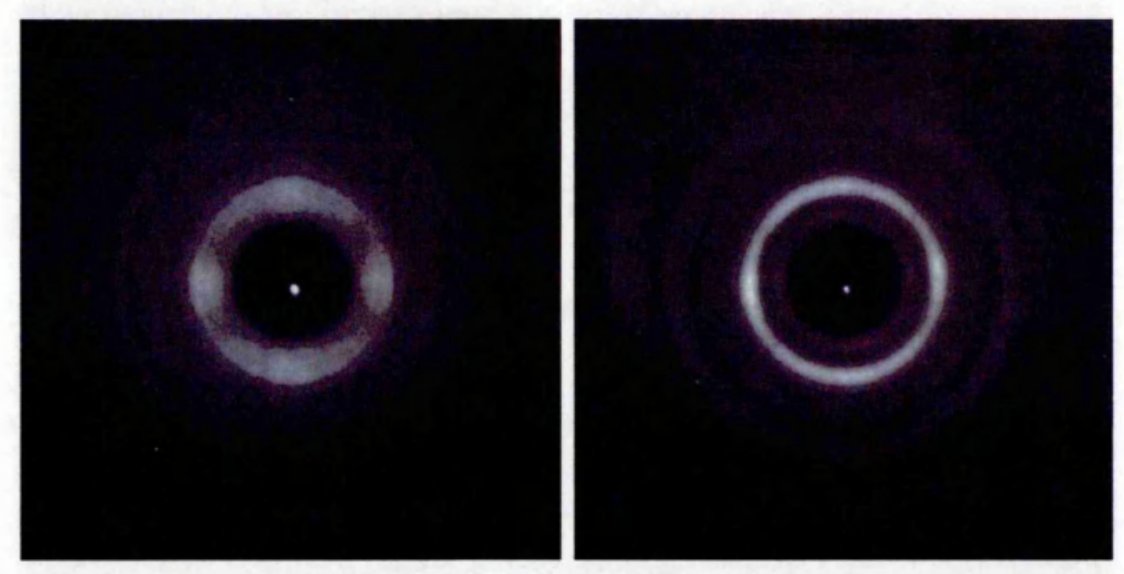

Fig. 3. Diffraction rings of ferrite steel before $\mathrm{X}$-ray beam filtering, and with filtering using $250 \mu \mathrm{m}$ of hafnium. 


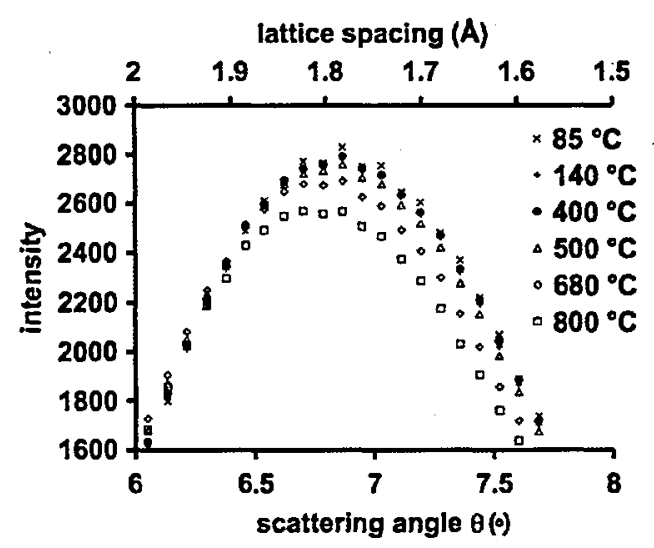

Fig. 5. Scattering angle of the $59.3 \mathrm{keV} \mathrm{X-rays} \mathrm{and} \mathrm{the} \mathrm{associated} \mathrm{lattice}$ spacing for a $1.2 \mathrm{~mm}$ thick ferrite steel sample subjected to annealing temperatures of $85^{\circ} \mathrm{C}, 140^{\circ} \mathrm{C}, 400^{\circ} \mathrm{C}, 500^{\circ} \mathrm{C}, 680^{\circ} \mathrm{C}$ and $800^{\circ} \mathrm{C}$.

resolving the lattice spacings, the system is better calibrated using the two extreme phases. The data presented in Fig. 5 shows the change in lattice spacing as a ferrite sample is annealed. As seen in the calibration data, the intensity of the diffraction ring at $7^{\circ}$ is expected to decrease as the lattice is annealed from the body-centred cubic structure of the ferrite phase, to the face-centred cubic structure of the austenite phase. While the sample has not been heated above the phase transition temperature, the variation in scattering intensity and scattering angle follows the expected trend; increasing lattice spacing in conjunction with a decrease in the scatter intensity as a result of reduced mass density due to thermal expansion. This effect is independent of the decrease that would be observed due to the structural change from body-centred cubic to face-centred cubic and therefore is an important consideration for any online system.

\section{Conclusions}

It has been demonstrated that an angular dispersive $\mathrm{X}$-ray diffraction measurement using a quasi-monochromated source and inexpensive X-ray image intensifier, could be used on an online process line for checking the annealing of the ferrite phase of steel into the desired austenite phase. The system has been calibrated using the two extreme phase conditions rather than using the known lattice spacing, given that the angular and spatial resolution using this system are not sufficient to make precise measurements of the lattice spacings. The role of temperature variation on the scattering profile has also been demonstrated, necessitating the requirement for austenite and ferrite samples to be at the same temperature in order to avoid density factors in the resulting observation of the phase transformation.

\section{Acknowledgement}

We are grateful to the British Steel PLC for providing the initial samples and to the Defence Procurement Agency for providing sponsorship for D.J. Garrity.

\section{References}

[1] R.D. Luggar, J.A. Horrocks, R.D. Speller, R.J. Lacey, Appl. Radiat. Isot. 48 (2) (1997) 215.

[2] J.H. Hubbell, W.J. Veigele, E.A. Briggs, R.T. Brown, D.T. Cromer, R.J. Howerton, J. Phys. Chem. Ref. Data 4 (3) (1975) 471.

[3] R.D. Luggar, W.B. Gilboy, Radiat. Phys. Chem. 56 (1999) 213.

[4] P.M. Jenneson, R.D. Luggar, E.J. Morton, O. Gundogdu, U. Tuzun, J. Appl. Phys 96 (5) (2004) 2889.

[5] JCPDS, in: W.F. mcClune (Ed.), International Centre for Diffraction Data, 1998, Pennsylvania, USA. 


\section{Appendix C}

\section{Further developments in transmission XRD}

Publication in Nucl. Instr. and Meth $A$ as part of the proceedings of the 10th International Symposium on Radiation Physics (ISRP-10). 


\title{
Transmission geometry X-ray diffraction for materials research
}

\author{
D.J. Garrity, P.M. Jenneson*, S.M. Vincent \\ Department of Physics, School of Electonics and Physical Sciences, University of Surrey, Stag Hill, Guildford, Surrey, GU2 $7 X H$, UK
}

Available online 16 May 2007

\begin{abstract}
A study of both Energy Dispersive X-ray diffraction (EDXRD) and Angular Dispersive XRD (ADXRD) in a transmission geometry, for materials research purposes has been carried out. Both techniques are sufficiently sensitive to observe structural changes that occur in steel, whether through heating of a sample to induce a phase transformation, or through plastic deformation. EDXRD using HPGe detectors provides the 'gold standard' for measurements under laboratory conditions. The information retrievable with the relaxed resolution of a Cadmium-Zinc-Telluride (CZT) detector compares favourably to that of the HPGe. An ADXRD measurement using a more robust apparatus, suitable for more challenging environmental conditions, is sufficiently sensitive to observe the ferrite to austenite phase transformation.

(C) 2007 Elsevier B.V. All rights reserved.
\end{abstract}

PACS: 61.10.Nz; 61.82.Bg

Keywords: Transmission X-ray diffraction; Stecl phases

\section{Introduction}

Coherent (Rayleigh) scattering of X-rays is increasingly used as a non-invasive probe for examining the nature of bulk materials. Over the last 10 years, coherent scattering of X-rays has been exploited for a broad range of applications [1] including industrial process control and aviation security. In the work reported here both Energy Dispersive X-ray diffraction (EDXRD) and Angular Dispersive XRD (ADXRD) techniques are being developed for measurement of phase transformations in steel.

Transmission ADXRD has been utilised to measure the ferrite to austenite phase transformation that would take place during annealing in a working steel foundry hot roller process line [2]. While both ADXRD and EDXRD ultimately provide the same information, the former technique has the advantage of utilising more robust equipment (a position sensitive detector and X-ray filtering) than the

\footnotetext{
${ }^{*}$ Corresponding author. Tel.: + 44 1483689419; fax: + 441483686781 .

E-mail address: p.jenneson@surrey.ac.uk (P.M. Jenneson).
}

EDXRD method, at the expense of the resolution obtainable with the system.

EDXRD requires a high resolution energy-resolving detector such as a hyper-pure germanium (HPGe), to produce high quality scatter spectra from the sample of interest and is being developed to look at the martensite phase transformation in austenitic stainless steel. HPGe detectors are however, delicate detectors requiring bulky cryogenic cooling systems. Small peltier-cooled Cadmium-Zinc-Telluride (CZT) detectors may offer a suitable compromise between resolution and the robustness of the experiment apparatus.

\section{Theory}

Coherent (elastic) scattering of electromagnetic waves from a single unbound electron is described classically by the Thomson scattering cross-section. For multiple, atomic electrons, the modified (Rayleigh) scattering cross-section is based upon the form factor approximation [3]. The form factor, $F[q, Z]$, is a momentum-space Fourier transform relating the Rayleigh scattering cross-section for bound 
electrons, to the classical Thompson scattering crosssection for a single unbound electron. The momentum transfer, $q$, is given by

$q=2 \hbar k \sin (\theta / 2)$.

Thus $q$ and by extension, the scattering amplitude, is dependent on the energy of the incoming photon and the scattering angle. Angular dispersive measurements utilise a fixed-wavelength source with a two-dimensional positionsensitive detector to investigate all regions of low momentum transfer (low scattering angles), producing a characteristic signature of the material under investigation. Energy dispersive measurements require a polychromatic source (the bremsstrahlung continuum of an industrial $X$-ray tube) with an energy-resolving detector to produce an cnergy spectrum at a single fixed angle.

With an ordered periodic array such as steel, scattering gives rise to a series of sharp peaks as associated with Bragg diffraction. Rather than calibrating the resultant spectra in terms of energy it is more useful to calibrate in terms of the parameter $x$ which is usually given in units of $\AA^{-1}$

$x=\frac{1}{i} \sin (\theta / 2)$.

Thus having performed the calibration in terms of $x$, the lattice spacing $d$ may be obtained simply from $x=\frac{1}{2} d$. In angular dispersive measurements scattering over all angles simultaneously produces a characteristic diffraction image corresponding to regions of constructive/destructive interference. In order to obtain the characteristic signature, a radial integration using the centre of the diffraction image pattern as the origin generates a profile in terms of 0 versus intensity, which should then be plotted in terms of $x$ as described above.

\section{Experiment apparatus}

Both ADXRD and EDXRD measurements have been carried out in a dedicated lead-shielded X-ray room with all equipment remotely operated from an adjacent control room. The experimental arrangement used for transmission ADXRD studies utilised a Thomson TH9428HP X-ray image intensificr. For EDXRD measurements, both a thin beryllium-window HPGe detector and Amptek XR-100 T CZT detector have been used in conjunction with a fixed collimator apparatus as shown in Fig. 1.

The X-ray source used was a $225 \mathrm{kVp}$ tungsten reflection target X-ray tube (Comet MXR225/22 powered by a Gulmay CP-225 supply) mounted inside a lead-lined enclosure in order to remove stray scatter produced from the X-ray tube, therefore ensuring that the resultant measured scattering was produced only from the sample being studied. The X-ray tube was operated at a potential of $100 \mathrm{kVp}$ throughout, with inherent filtration provided by an output window consisting of a $20 \mathrm{~mm}$ diameter, $1 \mathrm{~mm}$ thick disk of beryllium.

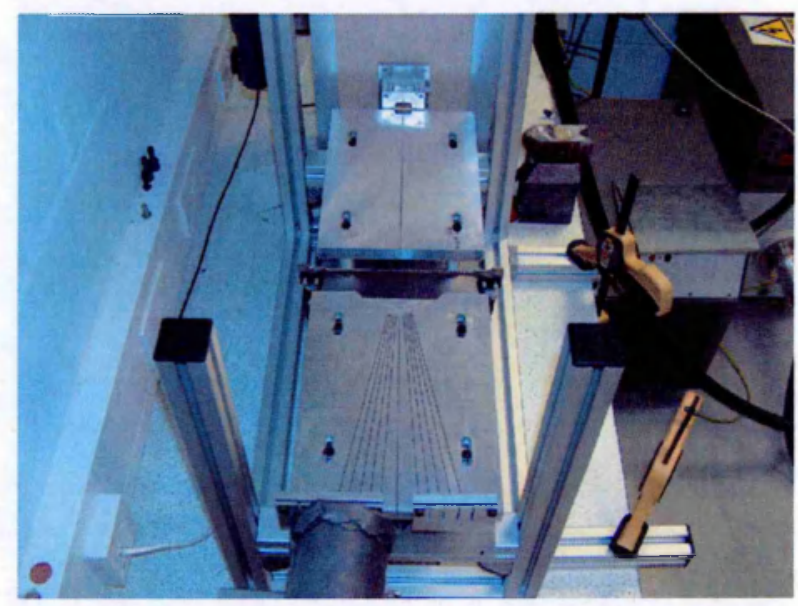

Fig. 1. The energy dispersive $X$-ray diffraction apparatus.

Primary source collimation for EDXRD work consists of an initial cylindrical, lead collimator with a $10 \mathrm{~mm}$ diameter bore and a tungsten-bladed cross-slit screen (HUBER Diffraktionstechnik 3013 ) to produce a $1 \times 10 \mathrm{~mm}$ ribbon beam. The steel samples under investigation are mounted, using optical filter mounts, into the centre of an aluminium collimator apparatus that provides both further incident X-ray collimation and collimation at fixed angles from $3^{\circ}$ to $12^{\circ}$, for elastically scattered X-rays. For this range of X-ray energies (up to $100 \mathrm{keV}$ ), a scattering angle of 9 degrees was found to be optimal for resolving the austenite lattice spacings and has been used throughout. In addition to the ADXRD measurements of the ferrite to austenite phase transformation, a series of plastically deformed austenite samples have been investigated using the EDXRD technique. Initial sample length for each sample was $19.7 \mathrm{~cm}$. The samples obtained consisted of lengths 20.01, 20.7, 21.4 and $26.5 \mathrm{~cm}$. Resulting lattice spacing measurements have been compared to the data from the powder diffraction file [3]. Measurements were initially carried out with the HPGe detector and later repeated with the more compact CZT detector.

\section{Results and discussion}

\subsection{Measurements of stressed austenitic stainless steel samples using EDXRD}

Measurements across the full range of samples revealed no increase in the lattice spacings themselves with increasing sample stress, which would have manifested as a peaks shifting towards lower energies. This in itself corresponds to slip deformation of the sample, i.e. movement of the parallel crystal planes without altering the crystal orientation or lattice spacing. As seen in Fig. 2, there is however, a noticeable change in the relative peak intensities corresponding to the $d_{220}$ and $d_{222}$ lattice 

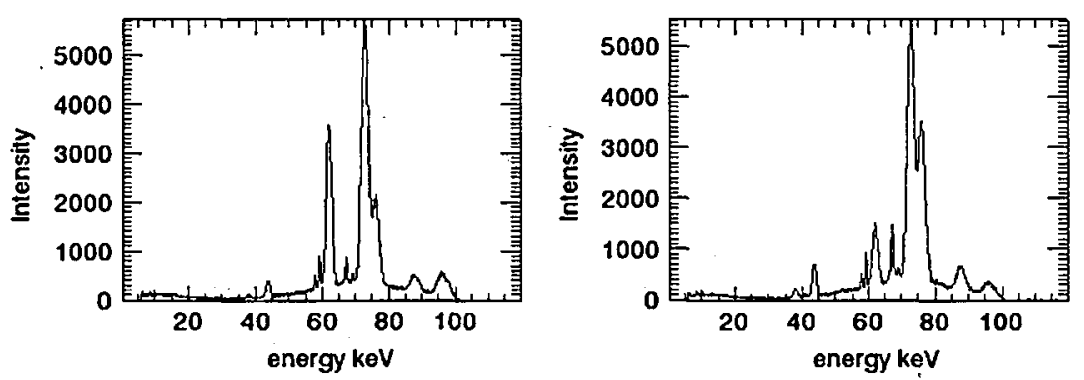

Fig. 2. Diffraction data obtained from samples of length 19.7 (left) and $21.4 \mathrm{~cm}$ (right) for a 9 degree scattering angle.
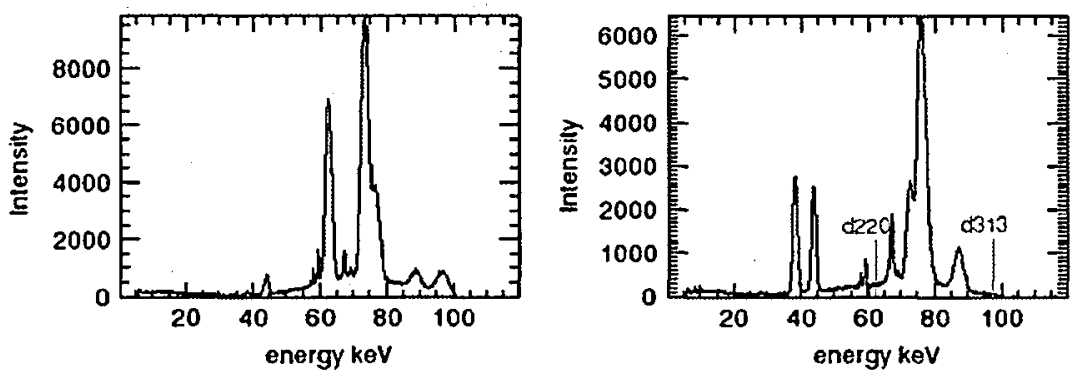

Fig. 3. Comparison of diffraction data obtained from the unstressed end of the $26.5 \mathrm{~cm}$ sample (left) to the centre of the sample (right) using the HPGe detector at a scattering angle of 9 degrees.
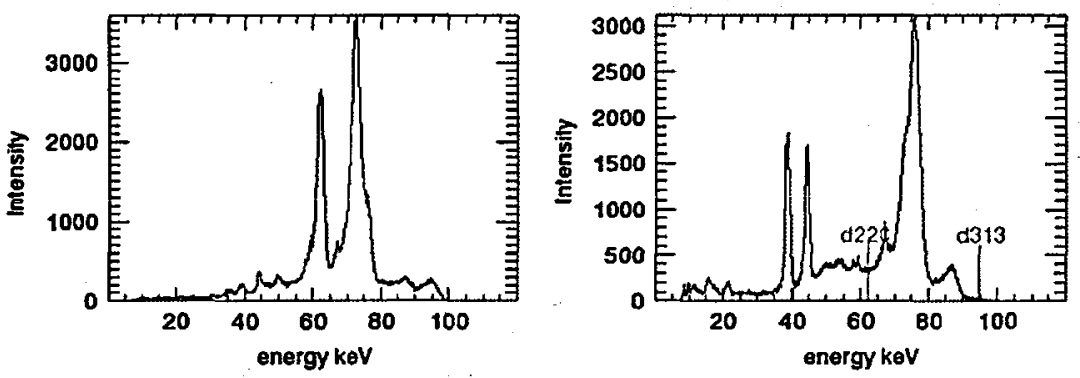

Fig. 4. Diffraction data for the unstressed cnd of $26.5 \mathrm{~cm}$ sample (left) and centre of the sample (right) as measured using the CZT detector at the 9 degree scattering angle.

spacings, the former decreasing in intensity while the latter increases in intensity.

Of interest is the $26.5 \mathrm{~cm}$ sample where a series of measurements have been taken across the full sample length. Fig. 3 compares the measurement taken at the centre of the sample (where the onset of failure is evident) to the unstressed end of the sample. What is striking is the loss of peaks corresponding to the $d_{313}$ and $d_{220}$ spacings, which may possibly be the result of a phase transformation from the face-centred cubic structure (FCC) of austenite to body-centred cubic (BCC) structure of martensite which can occur in this particular alloy under an applied strain $[4,5]$.

The measurements have been repeated with a CZT detector in order to determine if the information obtainable with the reduced energy resolution is comparable to that obtainable with the HPGe. Fig. 4 compares the centre and end measurements of the $26.5 \mathrm{~cm}$ deformed sample. Once again the loss of peaks corresponding to the $d_{313}$ and $d_{220}$ spacings is clearly resolvable; suggesting the lower energy resolution of detector is not a limiting factor in this type of measurement and CZT detectors may be useful for measurements outside of laboratory conditions.

\subsection{Measurements of the ferrite to austenite transformation using $A D X R D$}

Ferrite (mild steel) and austenite (stainless steel) samples have been previously analysed using the transmission ADXRD apparatus. Austenite phase steel is produced through heating ferrite steel above the phase transformation temperature, changing the structure from $\mathrm{BCC}$ to FCC. 

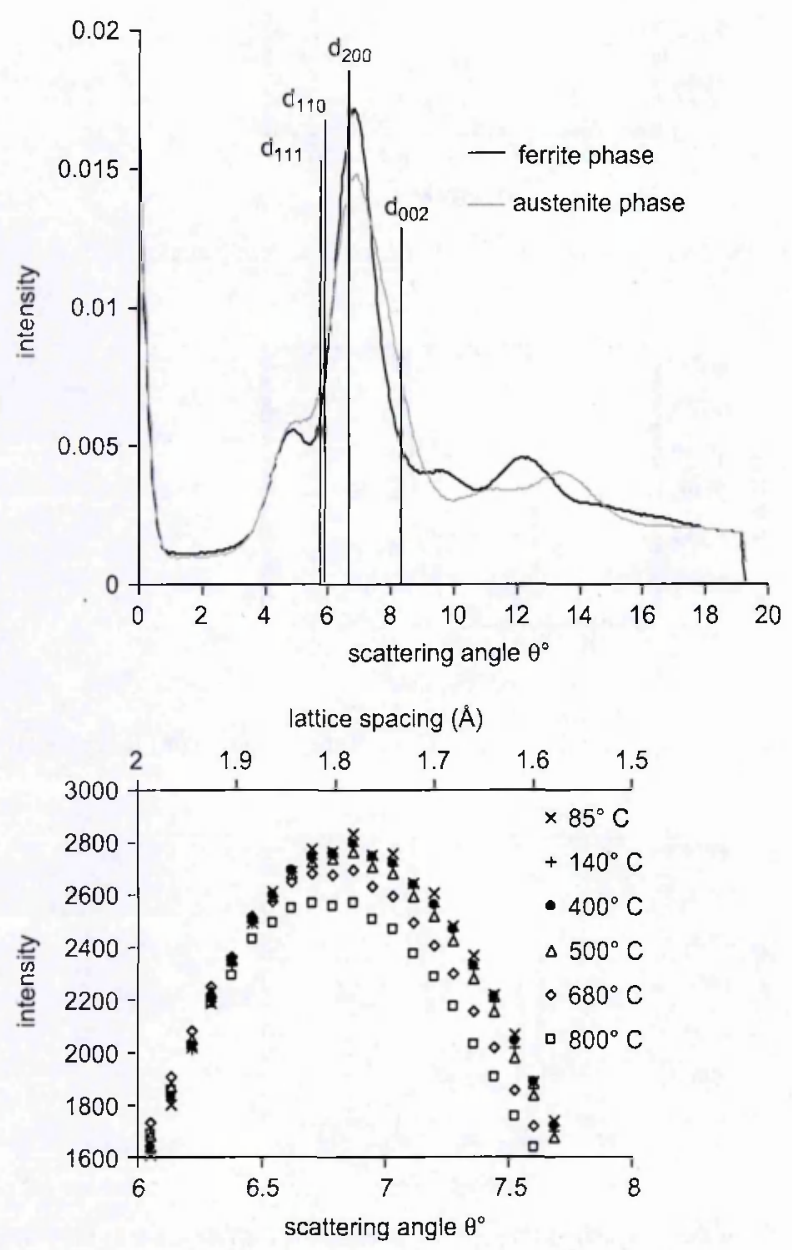

Fig. 5. (Top) calibration data obtained from the extreme ferrite and austenite phases, including positions of the $d_{111} d_{110} d_{200}$ and $d_{002}$ lattice spacings. (Bottom) diffraction data obtained for sample temperatures up to $800^{\circ} \mathrm{C}$.

To determine if the phases could be adequately resolved using transmission ADXRD a calibration measurement was performed using diffraction data obtained from the extreme ferrite and austenite phases as shown in Fig. 5. As a result of this calibration measurement it was determined the region of most interest would be between $6^{\circ}$ and $8^{\circ}$. Measurements of the diffraction pattern while heating a ferrite sample towards the phase transformation temperature yielded results in line with expectation; an increase in the measured lattice spacings with increasing temperature and a corresponding decrease in intensity, the result of reduced mass density due to the thermal expansion.

\section{Conclusions}

A comprehensive study of transmission geometry XRD has been carried out using both angular dispersive and energy dispersive configurations, demonstrating the sensitivity to structural changes induced in samples of steel whether through heating of a sample to induce a phase transformation, or plastic deformation. Energy dispersive measurements using a HPGe detector provide the 'gold standard' in terms of lattice spacing mcasurements under laboratory conditions. However, robust Peltier-cooled CZT detectors have proven sufficiently capable of imaging the same structural changes, without the requirement for bulky cryogenic cooling, thus making it a possible candidate for use outside of laboratory conditions.

Angular dispersive measurements are capable of detecting phase transformations with suitable calibration of the extreme phases. While the angular and spatial resolution of the system are not sufficient to make precise measurements of the lattice spacings, the apparatus is more suitable for use in extreme environmental conditions, for cxample, those found next to a steel foundry hot roller process line.

\section{References}

[1] R.D. Luggar, W.B. Gilboy, Radiat. Phys. Chem 56 (1999) 213.

[2] D.J. Garrity, P.M. Jenneson, R.D. Luggar, S.M. Vincent, Nucl. Instr. and Meth B 251 (2006) 197.

[3] W.F. McClunc, (Ed.), JCPDS Powder Diffraction File, Compiled by the JCPDS, International Centre for Diffraction Data, Pennsylvania, USA.

[4] M.C. Mataya, J.A. Lichenfield, C.J. Van Tyne, Metall. Mater. Trans. A 37 (1) (2006) 147.

[5] N.R. Bandyopadhyay, B.R. Kumar, B. Mahato, D.K. Bhattacharya, Metall. Mater. Trans. A 36 (11) (2005) 3165. 


\section{Bibliography}

[1] W. C. Röentgen, Sitzungsberichte der Physikalisch-medizinischen Gesellschaft zu Würzburg, 137 (1895).

[2] J. J. Thomson, Conductivity of electricity through gases, Cambridge University Press, 2nd ed., (1906).

[3] P. C. Johns and M. J. Yaffe, Med. Phys, 10, 40-50 (1983).

[4] R. D. Luggar, J. A. Horrocks, R. D. Speller, and R. J. Lacey, Appl. Radiat. Isot, 48(2), 215-224 (1997).

[5] C. H. Malden and R. D. Speller, Nucl. Instr. and Meth A, 449, 408-415 (2000).

[6] G. Harding and B. Schreiber, Radiat. Phys. Chem, 71, 869-881 (1999).

[7] M. J. Farquharson, R. D. Luggar, and R. D. Speller, Appl. Radiat. Isot, 48, 1075-1082 (1997).

[8] R. D. Luggar, M. J. Key, E. J. Morton, and W. B. Gilboy, Nucl. Instr. and Meth A, 422, 938-941 (1999). 
[9] R. D. Luggar and W. B. Gilboy, Radiat. Phys. Chem, 56, 213-227 (1999).

[10] G. Harding, J. Kosanetzky, and U. Neitzel, Med. Phys, 14, 515-525 (1987).

[11] S. Pani, 7th International Position Sensitive Detectors Conference, (2005).

[12] C. R. F. Castro, R. C. Barroso, L. F. de Oliveira, and R. T. Lopes, Nucl. Instr. and Meth $A, 548,116-122$ (2005).

[13] R. E. Smallman and R. J. Bishop, Metals and Materials: Science, Processes, Applications, Butterworth-Heinemann Ltd, (1995).

[14] R. D. Luggar and P. M. Jenneson, Commercially confidential, (2000).

[15] J. W. Strutt, Phil. Mag., 41, 107-120 (1871).

[16] D. R. Hartree, Phil. Mag., 50, 89-132 (1925).

[17] W. Franz, Zëitschrift für Physik, 95, 652-668 (1935).

[18] L. Pauling and J. Sherman, Zëitschrift für Kristallographie, 81, 1-29 (1932).

[19] O. Klein and Y. Nishina, Zëitschrift für Physik, 52, 853 (1929).

[20] M. Laue, Proc. Acad. Bavarian Sci., 363-373, (1912).

[21] W. Friedrich, P. Knipping, and M. Laue, Proc. Acad. Bavarian Sci., 303-322, (1912). 
[22] W. H. Bragg and W. L. Bragg, Proc. Roy. Soc. A, 88, 428-438 (1913).

[23] J. D. Jackson, Classical Electrodynamics, Wiley, 3rd ed., (1999).

[24] D. J. Griffiths, Introduction to Electrodynamics, Prentice Hall, 3rd ed., (1999).

[25] R. M. Eisberg, Quantum physics of atoms, molecules, solids, nuclei and particles, Wiley, 2nd ed., (1985).

[26] P. P. Kane, L. Kissel, R. H. Pratt, and S. C. Roy, Phys. Rep, 140(2), 75-159 (1986).

[27] NIST Physics laboratory database, http://physics.nist.gov/, (2005).

[28] D. A. Bradley, O. D. Gonçalves, and P. P. Kane, Radiat. Phys. Chem; 56(1), 175-195 (1999).

[29] JCPDS (1998) Powder Diffraction file (compiled by the JCPDS, International Centre for Diffraction Data), Editor W. F. McClune, Pennsylvania, USA.

[30] K. S. Krane, Introductory Nuclear Physics, Wiley, (1988).

[31] European Synchrotron Radiation Facility, http://www.esrf.eu/Accelerators/Performance/Brilliance, (2007).

[32] Deutsches Elektronen-Synchrotron (DESY), http://www.desy.de, (2007).

[33] P. M. Jenneson, R. D. Luggar, E. J Morton, O. Gundogdu, and U. Tuzun, J. Appl. Phys, 96(5), 2889-2894 (2004). 
[34] IPEM Report 87, (1997), Catalogue of Diagnostic X-Ray Spectra and Other Data, Institute of Physics and Engineering in Medicine.

[35] D. J. Garrity, P. M. Jenneson, S. M. Vincent and A. C. Williams, Unpublished MoD report, Determination of X-ray Tube Stability for use in X-ray Transmission Diffraction Research, (2005).

[36] D. J. Garrity, P. M. Jenneson, R. D. Luggar, and S. M. Vincent, Nucl. Instr. and Meth B, 251, 197-200 (2006).

[37] B. E. Stern and D. Lewis, X-rays, Pitman, (1970).

[38] C. G. Barkla and C. A. Sadler, Phil. Mag., 14, 408-422 (1907).

[39] CERN, Medipix2 Collaboration, http://medipix.web.cern.ch/MEDIPIX/, (2005).

[40] EECA market report 2005 summer, http://eeca.org.

[41] G. F. Knoll, Radiation Detection and Measurement, Wiley, 3rd ed., (2000).

[42] A. Lohstroh, M. E. Özsan, and P. J. Sellin, Nucl. Instr. and. Meth A, 546(1-2), 192-199 (2005).

[43] K. Suzuki, S. Seto, A. Tanaka, and M. Kawashima, Journal of Crystal Growth, 101(1-2), 859-863 (1990).

[44] Amptek X-ray and Gamma ray detector XR-100T CdTe, http://www.amptek.com/xrcdtaps.html, (2007). 
[45] Amptek XR-100T CdTe/CZT Efficiency Note, http://www.amptek.com/anczt1.html, (2007).

[46] Ortec GLP Series Planar Germanium (HPGe) Low-Energy Photon Detectors, http://www.ortec-online.com/, (2007).

[47] J. A. Lichtenfeld, M. C. Mataya, and C. J. Van Tyne, Metall. Mater. Trans. A, Phys. Metall, Matar. Sci (USA), 37(1), 147-162 (2006).

[48] B. R. Kumar, B. Mahato, N. R. Bandyopadhyay, and D. K. Bhattacharya, Metall. Mater. Trans. A, Phys. Metall, Matar. Sci (USA), 36(11), 3165-3174 (2005).

[49] S. E. Jarmen, Private communication, (2007).

[50] D. J. Garrity, P. M. Jenneson, and S. M. Vincent, Nucl. Instr. and Meth $A, 580,412-415$ (2007).

[51] Metropolitan Museum of Art, New York;

The Japanese Blade: Technology and Manufacture, http://www.metmuseum.org/toah/hd/japb/hd_japb.htm, (2006).

[52] H. K. D. H. Bhadeshia, Worked examples in the Geometry of Crystals, The Institute of Materials, 2nd ed., (2001).

[53] SIMULIA, Dassault Systemes, http://www.simulia.com, (2006).

[54] M. Wenman and A. Price, Private communication, (2006).

[55] M. R. Daymond, C. N. Tome, and M. A. M. Bourke, Acta Materialia, $48,553-564$ (2000). 
[56] A. Price, M. Wenman, experiment MA-181, ID15A beamline, European Synchrotron Radiation Facility, (2006).

[57] A. Price, Private communication, (2007).

[58] E. Cabruja, M. Bigas, M. Ullan, G. Pellegrini, and M. Lozano, Nucl. Instr. and Meth $A, \mathbf{5 7 6}, 150-153$ (2007).

[59] G. Pellegrini, M. Maiorino, G. Blanchot, M. Chmeissani, J. Garcia, M. Lozano, R. Martinez, C. Puigdengoles, and M. Ullan, Nucl. Instr. and Meth $A, \mathbf{5 7 3}, 137-140$ (2007).

[60] E. Heijne, 7th International Position Sensitive Detectors Conference, (2005).

[61] Dectris PILATUS 6M Macromolecular Crystallography detector system, http://www.dectris.com/sites/pilatus6m.html, (2007).

[62] SLS detector group, http://pilatus.web.psi.ch/pilatus.htm, (2007). 